ANALYSIS \& PDE Volume $6 \quad$ No. $5 \quad 2013$

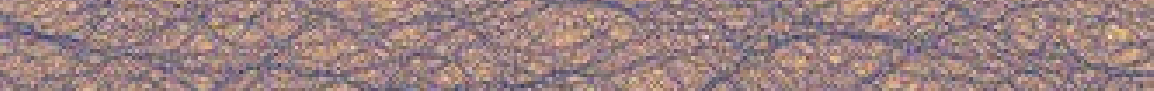
JUHI JANG AND IAN TICE

INSTABILITY THEORY OF THE NAVIER-STOKES-POISSON EQUATIONS 


\title{
INSTABILITY THEORY OF THE NAVIER-STOKES-POISSON EQUATIONS
}

\author{
JUHI JANG AND IAN TICE
}

\begin{abstract}
The stability question of the Lane-Emden stationary gaseous star configurations is an interesting problem arising in astrophysics. We establish both linear and nonlinear dynamical instability results for the Lane-Emden solutions in the framework of the Navier-Stokes-Poisson system with adiabatic exponent $\frac{6}{5}<\gamma<\frac{4}{3}$.
\end{abstract}

\section{Introduction and formulation}

One of the simplest fundamental hydrodynamical models to describe the motion of self-gravitating viscous gaseous stars is the compressible Navier-Stokes-Poisson system, which can be written in Eulerian coordinates as

$$
\left\{\begin{array}{l}
\partial_{t} \varrho+\operatorname{div}(\varrho \boldsymbol{u})=0, \\
\partial_{t}(\varrho \boldsymbol{u})+\operatorname{div}(\varrho \boldsymbol{u} \otimes \boldsymbol{u})+\operatorname{div} S=-\varrho \nabla \Phi, \\
\Delta \Phi=4 \pi \varrho,
\end{array}\right.
$$

where $(\boldsymbol{x}, t) \in \mathbb{R}^{3} \times \mathbb{R}^{+}, \varrho(\boldsymbol{x}, t) \geq 0$ is the density, $\boldsymbol{u}(\boldsymbol{x}, t) \in \mathbb{R}^{3}$ is the velocity vector field of the gas, $\Phi(\boldsymbol{x}, t) \in \mathbb{R}$ is the potential function of the self-gravitational force, and the stress tensor $S$ is given by

$$
S=P I_{3 \times 3}-\varepsilon\left(\nabla \boldsymbol{u}+\nabla \boldsymbol{u}^{t}-\frac{2}{3}(\operatorname{div} \boldsymbol{u}) I_{3 \times 3}\right)-\delta(\operatorname{div} \boldsymbol{u}) I_{3 \times 3},
$$

where $P$ is the pressure of the gas, $\varepsilon>0$ is the shear viscosity, $\delta \geq 0$ is the bulk viscosity, and $\nabla \boldsymbol{u}^{t}$ denotes the transpose of $\nabla \boldsymbol{u}$. We consider polytropic gases for which the equation of state is given by

$$
P=P(\varrho)=K \varrho^{\gamma},
$$

where $K$ is an entropy constant and $\gamma>1$ is an adiabatic exponent. Values of $\gamma$ have their own physical significance [Chandrasekhar 1939]; for instance, $\gamma=\frac{5}{3}$ corresponds to a monatomic gas and $\gamma=\frac{7}{5}$ to a diatomic gas, and $\gamma \rightarrow 1^{+}$for heavier molecules.

In the simplest setting, which we consider, solutions to (1-1) are spherically symmetric. For $r=|\boldsymbol{x}|$, this allows us to write

$$
\boldsymbol{u}(\boldsymbol{x}, t)=u(r, t) \frac{\boldsymbol{x}}{r} \text { for } u:[0, \infty) \times[0, \infty) \rightarrow \mathbb{R}
$$

Jang was supported by NSF grants DMS-0908007 and DMS-1212142. Tice was supported by an NSF Postdoctoral Research Fellowship.

MSC2010: 35Q30, 35R35, 76E20, 85A30.

Keywords: Navier-Stokes-Poisson, free boundary problems, gaseous stars, hydrodynamic instability, nonlinear instability. 
and

$$
\varrho(\boldsymbol{x}, t)=\varrho(r, t) .
$$

The equations (1-1) then reduce to the pair

$$
\partial_{t} \varrho+u \partial_{r} \varrho+\frac{\varrho}{r^{2}} \partial_{r}\left(r^{2} u\right)=0
$$

and

$$
\varrho\left(\partial_{t} u+u \partial_{r} u\right)+\partial_{r} P=-\frac{4 \pi \varrho}{r^{2}} \int_{0}^{r} \varrho(s, t) s^{2} d s+\partial_{r}\left(\frac{\frac{4}{3} \varepsilon+\delta}{r^{2}} \partial_{r}\left(r^{2} u\right)\right) .
$$

The integral term on the right side of (1-7) corresponds to the gravitational force. Stationary solutions $\varrho=\varrho_{0}(r)$ and $u=0$, which correspond to nonmoving gaseous spheres in hydrostatic equilibrium, satisfy the following equation for $P_{0}=K \varrho_{0}^{\gamma}$ :

$$
\partial_{r} P_{0}(r)+\frac{4 \pi \varrho_{0}(r)}{r^{2}} \int_{0}^{r} \varrho_{0}(s) s^{2} d s=0 .
$$

This equation can be solved by transforming it into the well-known Lane-Emden equation [Chandrasekhar 1939]. The solutions to (1-8) are positive and decreasing and can be characterized by the values of $\gamma$ in the following fashion [Lin 1997]: for given finite total mass $M>0$, if $\gamma \in\left(\frac{6}{5}, 2\right)$, there exists at least one compactly supported solution $\varrho_{0}$. For $\gamma \in\left(\frac{4}{3}, 2\right)$, every solution is compactly supported and unique. If $\gamma=\frac{6}{5}$, the unique solution admits an analytic expression, and it has infinite support. On the other hand, for $\gamma \in\left(1, \frac{6}{5}\right)$, there are no solutions with finite total mass.

The stability of the Lane-Emden steady star configurations has been a question of great interest, and it has been conjectured by astrophysicists that stationary solutions for $\gamma<\frac{4}{3}$ are unstable. The linear stability theory of the above stationary solutions was studied in [Lin 1997] in the inviscid case, namely the Euler-Poisson system, by studying the eigenvalue problem associated to the linearized Euler-Poisson system: any stationary solution is linearly stable when $\gamma \in\left(\frac{4}{3}, 2\right)$ and unstable when $\gamma \in\left(1, \frac{4}{3}\right)$. In accordance with the linear stability theory, a nonlinear stability for $\gamma>\frac{4}{3}$ was established in [Rein 2003] by using a variational approach. In the case $\gamma=\frac{4}{3}$, the analysis in [Deng et al. 2002] identified an instability in which any small perturbation can cause part of the system to go off to infinity. In [Jang 2008], a nonlinear instability of the Lane-Emden steady star for $\gamma=\frac{6}{5}$ was proved based on the bootstrap argument, as pioneered in [Guo and Strauss 1995]. The stability question for the Euler-Poisson system with $\frac{6}{5}<\gamma<\frac{4}{3}$ remains an open problem.

The same stability question can also be asked in the presence of viscosity. There have been interesting studies on the stabilization effect of viscosity in the Navier-Stokes-Poisson system for $\gamma>\frac{4}{3}$ under various assumptions [Ducomet and Zlotnik 2005; Zhang and Fang 2009]. On the other hand, to our knowledge, no rigorous stability theories are available for $\gamma<\frac{4}{3}$, the instability regime in the inviscid case. In this regime for viscous gaseous stars, a particularly interesting problem is to investigate whether or not the viscosity would dominate the gravitational force and stabilize the whole system. The purpose of this article is to establish the instability theory of the Lane-Emden steady stars whose dynamics are governed by the Navier-Stokes-Poisson system for $\frac{6}{5}<\gamma<\frac{4}{3}$. 
We now formulate the problem. We begin by introducing a vacuum free boundary.

1A. Vacuum free boundary. When $\gamma>\frac{6}{5}$, letting $R>0$ be the radius of the steady star, it is well known [Lin 1997] that

$$
\varrho_{0}(r) \sim(R-r)^{1 /(\gamma-1)} \quad \text { for } r \text { near } R
$$

This boundary behavior near a vacuum causes a degeneracy in (1-6) and (1-7), and it is not trivial to deal with such a degeneracy even for the local-in-time existence question; we refer, for instance, to [Jang 2010; Matusu-Necasova et al. 1997; Okada and Makino 1993] and also [Jang and Masmoudi 2009; 2010] for the compressible Euler case. It turns out that in order to capture boundary behavior such as (1-9) in the dynamical setting, one has to consider a free boundary problem associated to (1-6) and (1-7) as in [Jang 2010; Matusu-Necasova et al. 1997; Okada and Makino 1993]. We are interested in the evolution of compactly supported stars with a free boundary where the star meets a vacuum. This is implemented by assuming there is a radius $R=R(t)>0$ such that

$$
\varrho(r, t)>0 \quad \text { for } r \in[0, R(t)) \quad \text { and } \quad \varrho(R(t), t)=0 .
$$

At the free boundary we impose the kinematic condition

$$
\frac{d}{d t} R(t)=u(R(t), t)
$$

as well as the continuity of the normal stress, $S v=0$ at the surface $r=R(t)$. The latter condition reduces to

$$
P-\frac{4 \varepsilon}{3}\left(\partial_{r} u-\frac{u}{r}\right)-\delta\left(\partial_{r} u+\frac{2 u}{r}\right)=0 \quad \text { for } r=R(t), t \geq 0 .
$$

Note that $P(R(t), t)=K \varrho^{\gamma}(R(t), t)=0$, so this can be reduced to a relationship between $\partial_{r} u$ and $u$ at $r=R(t)$. Finally, in order for $\boldsymbol{u}=u(r, t) \boldsymbol{x} / r$ to be continuous, we require $u(0, t)=0$ for $t \geq 0$.

Since the boundary $R(t)$ is free to move in time in Eulerian coordinates, it is convenient to introduce Lagrangian coordinates so that the boundary becomes fixed. Following the framework used in [Jang 2010; Matusu-Necasova et al. 1997; Okada and Makino 1993], we study our instability problem in Lagrangian mass coordinates.

1B. Formulation in Lagrangian mass coordinates. We now reformulate the problem in Lagrangian mass coordinates. We set

$$
x(r, t)=\int_{0}^{r} 4 \pi s^{2} \varrho(s, t) d s=\int_{B(0, r)} \varrho(y, t) d y
$$

for the mass contained in an Eulerian ball of radius $r$ at time $t$. Note that

$$
\partial_{r} x(r, t)=4 \pi r^{2} \varrho(r, t)
$$

and that

$$
\partial_{t} x(r, t)=\int_{B(0, r)} \partial_{t} \varrho(y, t) d y=-\int_{B(0, r)} \operatorname{div}(\varrho \boldsymbol{u}) d y=-\int_{\partial B(0, r)} \varrho \boldsymbol{u} \cdot v=-4 \pi r^{2} \varrho(r, t) u(r, t) .
$$


In particular, this implies that $\partial_{t} x(R(t), t)=0$, which means that the total mass $M>0$ is preserved in time. The domain of $x$ is then $[0, M]$. Switching to Lagrangian mass coordinates $(x, t) \in[0, M] \times[0, \infty)$ and letting the unknowns be

$$
\rho(x, t)=\varrho(r, t) \quad \text { and } \quad v(x, t)=u(r, t),
$$

we get the equations

$$
\partial_{t} \rho+4 \pi \rho^{2} \partial_{x}\left(r^{2} v\right)=0
$$

and

$$
\partial_{t} v+4 \pi r^{2} \partial_{x} P+\frac{x}{r^{2}}=16 \pi^{2} r^{2} \partial_{x}\left(\left(\frac{4 \varepsilon}{3}+\delta\right) \rho \partial_{x}\left(r^{2} v\right)\right) .
$$

In Lagrangian coordinates, our boundary conditions reduce to

$$
v(0, t)=0, \quad \rho(M, t)=0,
$$

and

$$
P-\frac{4 \varepsilon}{3}\left(4 \pi r^{2} \rho \partial_{x} v-\frac{v}{r}\right)-\delta\left(4 \pi r^{2} \rho \partial_{x} v+\frac{2 v}{r}\right)=0 \quad \text { at } x=M \quad \text { for all } t \geq 0 .
$$

In each of these equations, we have written

$$
r(x, t)=\left(\frac{3}{4 \pi} \int_{0}^{x} \frac{d y}{\rho(y, t)}\right)^{1 / 3}
$$

which inverts (1-13) by way of integrating (1-14). A simple computation, employing (1-17), shows that $\partial_{t} r(x, t)=v(x, t)$.

A stationary solution $\rho=\rho_{0}(x), v=0, P_{0}=K \rho_{0}^{\gamma}$ to (1-17) and (1-18) satisfies the equation

$$
4 \pi r_{0}^{2}(x) \partial_{x} P_{0}(x)+\frac{x}{r_{0}^{2}(x)}=0
$$

where

$$
r_{0}(x)=\left(\frac{3}{4 \pi} \int_{0}^{x} \frac{d y}{\rho_{0}(y)}\right)^{1 / 3} .
$$

This is the Lagrangian version of (1-8). We denote such a Lane-Emden solution in Lagrangian mass coordinates by $\rho_{0}$ with pressure $P_{0}=K \rho_{0}^{\gamma}$. Note that $\rho_{0}(x)>0$ for $x \in[0, M)$ and that $\rho_{0}$ decreases until it vanishes at $x=M$. In Lagrangian $x$ coordinates, the boundary behavior (1-9) is expressed as

$$
\rho_{0}(x) \sim(M-x)^{1 / \gamma} \text { for } x \text { near } M,
$$

which can be also seen from (1-22). In particular, when $\gamma \in\left(\frac{6}{5}, \frac{4}{3}\right)$, this implies that $\frac{1}{\rho_{0}(x)}$ is integrable, so that $R=r_{0}(M)<\infty$, which corresponds to a star of finite radius.

The existence and uniqueness of strong solutions to the vacuum free boundary problem of the NavierStokes-Poisson system (1-17) and (1-18) featuring the behavior (1-24) of Lane-Emden solutions was established in [Jang 2010] when $\delta=2 \varepsilon / 3>0$. The same methodology can be applied to our current setting as long as $\varepsilon>0$ and $\delta>0$, and we will take those strong solutions for granted in proving our 
nonlinear instability result. A well-posedness result in our energy space can be also proved based on our new a priori energy estimates for the fully nonlinear Navier-Stokes-Poisson system, described in Section 4.

1C. Main results. Throughout the paper, we assume that

$$
\varepsilon>0, \quad \delta>0, \quad K>0, \quad \text { and } \quad \frac{6}{5}<\gamma<\frac{4}{3}
$$

are all fixed. Note that although the only physical requirement on the bulk viscosity is $\delta \geq 0$, the assumption $\delta>0$ is critical for both our linear and nonlinear analysis. We will also write $M, R>0$ for the mass and radius of a stationary solution to (1-22).

To state the main results, we first write the system in a perturbation form. For small perturbed solutions $\sigma:=\rho-\rho_{0}$ and $v$ around the steady states satisfying (1-22), the Navier-Stokes-Poisson system (1-17) and (1-18) can be written as

$$
\begin{gathered}
\partial_{t} \sigma+4 \pi \rho^{2} \partial_{x}\left(r^{2} v\right)=0 \\
\partial_{t} v+4 \pi r^{2} \partial_{x} P-4 \pi r_{0}^{2} \partial_{x} P_{0}+\frac{x}{r^{2}}-\frac{x}{r_{0}^{2}}=16 \pi^{2} r^{2} \partial_{x}\left(\left(\frac{4 \varepsilon}{3}+\delta\right) \rho \partial_{x}\left(r^{2} v\right)\right)
\end{gathered}
$$

with boundary conditions (1-19) and (1-20).

Our first main result concerns the existence of the largest growing mode of the linearized NavierStokes-Poisson system around Lane-Emden solutions, which shows a linear instability in the sense of Lin's stability criteria [1997].

Theorem 1.1. Suppose (1-25). There exist $\lambda>0$ and $\sigma(x), v(x)$ such that $\sigma(x) e^{\lambda t}$ and $v(x) e^{\lambda t}$ solve the linearized Navier-Stokes-Poisson system (2-1) and (2-2) with the linearized boundary conditions (2-3) and (2-4). Moreover, this growing mode yields the largest possible growth rate to the linearized system.

Remark 1.2. The growth rate $\lambda>0$ produced in Theorem 1.1 clearly depends on the values of the viscosity parameters $\varepsilon, \delta$. It is natural to consider the asymptotics of $\lambda$ for large and small viscosities. In Proposition 2.11 below, we show that $\lambda$ converges to the largest growth rate for the inviscid problem (identified by Lin [1997]) as $(\delta, \varepsilon) \rightarrow 0$. We also show that $\lambda \rightarrow 0$ as $\delta \rightarrow \infty$, which demonstrates that viscosity delays the onset of instability, since the escape time $T^{\iota}$ (given below in (1-27)) is inversely proportional to $\lambda$.

The precise statement of Theorem 1.1 with the estimates is given in Theorems 2.1 and 3.2. Our second main result establishes the fully nonlinear dynamical instability of the Lane-Emden solutions to the Navier-Stokes-Poisson system. In the statement of the theorem, for any given $\iota>0$ and $\theta>\iota$, we write

$$
T^{\iota}:=\frac{1}{\lambda} \ln \frac{\theta}{\iota},
$$

where $\lambda$ is the sharp linear growth rate obtained in Theorem 1.1.

Theorem 1.3. Suppose (1-25). There exist function spaces $X$ and $Y$ as well as constants $\theta>0$ and $C>0$ such that for any sufficiently small $\iota>0$, there exist solutions $\left(\sigma^{\iota}(t), v^{\iota}(t)\right)$ to (1-26) for $t \in[0, T)$ with 
$T>T^{\iota}$ such that

$$
\left\|\left(\sigma^{\iota}(0), v^{\iota}(0)\right)\right\|_{Y} \leq C \iota, \quad \text { but } \quad \sup _{0 \leq t \leq T^{\iota}}\left\|\left(\sigma^{\iota}(t), v^{\iota}(t)\right)\right\|_{X} \geq \theta .
$$

The precise statement of Theorem 1.3 is given in Theorem 5.4, and the spaces $X$ and $Y$ will be clarified in Sections 4 and 5.

Remark 1.4. Our results show that regardless of how large the viscosity parameters $\varepsilon, \delta$ are, and no matter how small smooth initial perturbed data are taken to be, the system remains unstable. We conclude from this that all Lane-Emden steady star configurations for $\frac{6}{5}<\gamma<\frac{4}{3}$ are unstable, regardless of viscosity.

Remark 1.5. The escape time $T^{\iota}$ is determined through (1-27) by the linear growth rate $\lambda$. We note that the instability occurs before the possible breakdown or any collapse of strong solutions. We also remark that the instability occurs in the $X$ norm, which when rewritten in Eulerian coordinates, is equivalent to

$$
\int_{\mathbb{R}^{3}}\left(\frac{1}{2} \varrho|\boldsymbol{u}|^{2}+\frac{\gamma}{2} \frac{\varrho_{0}}{\varrho} P_{0}\left|\frac{\sigma}{\varrho_{0}}\right|^{2}\right) d \boldsymbol{x},
$$

which is related to the positive part of the physical energy:

$$
\int_{\mathbb{R}^{3}}\left(\frac{1}{2} \varrho|\boldsymbol{u}|^{2}+\frac{1}{\gamma-1} P\right) d \boldsymbol{x} .
$$

Of course, this is not a coincidence: the Lane-Emden solutions for $\gamma<\frac{4}{3}$ do not minimize the physical energy functional,

$$
\begin{aligned}
& \int_{0}^{M}\left(\frac{1}{2}|v|^{2}+\frac{1}{\gamma-1} \frac{P}{\rho}-\frac{x}{r}\right) d x \text { in Lagrangian mass coordinates, or } \\
& \int_{\mathbb{R}^{3}}\left(\frac{1}{2} \varrho|\boldsymbol{u}|^{2}+\frac{1}{\gamma-1} P\right) d \boldsymbol{x}-\frac{1}{2} \int_{\mathbb{R}^{3} \times \mathbb{R}^{3}} \frac{\varrho(\boldsymbol{x}) \varrho(\boldsymbol{y})}{|\boldsymbol{x}-\boldsymbol{y}|} d \boldsymbol{x} d \boldsymbol{y} \text { in Eulerian coordinates, }
\end{aligned}
$$

and thus one might expect some kind of instability. They do minimize for $\gamma>\frac{4}{3}$ (see, for instance, [Jang 2008; Rein 2003]).

The presence of viscosity and the nonlinear boundary condition (1-20) for the Navier-Stokes-Poisson system make the problem distinguishable and interesting not only from a physical point of view, but also from a mathematical point of view. What follows now are some of the main mathematical difficulties we encounter in analyzing the system, and a brief discussion of our methods for resolving them.

The proof of Theorem 1.1 is based on a variational analysis of equations obtained by linearizing (1-26). The main difficulty that arises in constructing growing-mode solutions is that, due to the viscous terms, the growth rate (eigenvalue) appears in the problem with two different homogeneities. This breaks the natural variational structure used in [Lin 1997] to construct growing modes in the inviscid case. To get around this difficulty, we employ a technique introduced in [Guo and Tice 2010]: we introduce a relaxed parameter that allows us to remove one of the eigenvalue homogeneities, study the resulting modified eigenvalue problem (which has a nice variational structure), and finally return to the original formulation 
through a fixed point argument. While the solutions constructed in this manner are definitely growing modes, it is not clear a priori that they grow at the largest possible rate. To verify this, we carry out a careful analysis, paying particular attention to the boundary behavior of the growing mode, which will be crucially used in the subsequent nonlinear bootstrap argument.

The proof of Theorem 1.3 is based on a bootstrap argument from linear instability to nonlinear dynamical instability. Passing from a linearized instability to nonlinear instability requires much effort in the PDE context since the spectrum of the linear part is fairly complicated and the unboundedness of the nonlinear part usually yields a loss in derivatives. In order to get around these difficulties and to find the right space $Y$, we employ careful nonlinear energy estimates for the whole system so that, first, the nonlinear estimates can be closed, and second, their interplay with the linear analysis can complete the argument. For this particular problem, the space $Y$ is minimally chosen so that the viscosity disturbance near the vacuum boundary can be controlled within $Y$.

We note that in Lagrangian mass coordinates, the continuity equation interacts well with the viscosity term, which allows us to derive nice estimates for $\sigma / \rho_{0}$ and its temporal and spatial derivatives. This plays an important role in closing our nonlinear energy estimates. The main technical difficulty is to derive Proposition 5.1, a key estimate for the bootstrap argument. The idea is to find an energy $\mathscr{E}$ that satisfies an inequality of the form

$$
\frac{d}{d t} \mathscr{E} \leq \eta \mathscr{E}+\text { lower derivative terms, }
$$

where $\eta$ is smaller than the sharp linear growth rate. However, (1-32) is too good to hold in general due to the degeneracy of vacuum boundary and the complexity of the system near Lane-Emden stars. To overcome this difficulty, we introduce a collection of energy terms: some of them satisfy (1-32) under certain conditions, which we quantify; some of them are bootstrapped energies, the estimates of which are obtained by improved weighted energy estimates that exploit the structure of the equations; and others are auxiliary energies, the estimates of which are directly obtained from the equations. The gravitational potential has a smoothing effect, behaves well with the necessary weights, and does not create further difficulty in the nonlinear estimates. In fact, Section 4 is devoted to the introduction of those energy terms and the derivation of the estimates. Combining the estimates of the various energy terms, we can complete the bootstrap argument.

Another delicate and important issue is the nonlinear boundary condition (1-20). In order to carry out higher-order energy estimates that require integration by parts, we can only employ differential operators that respect the boundary conditions, namely temporal derivatives. This forces us to carefully use the structure of the equations in order to gain bounds on spatial derivatives. A second difficulty with the boundary arises because we use Duhamel's principle to study the nonlinear problem with the linearized evolution operator. The linearized boundary condition is homogeneous, but the nonlinear boundary condition is certainly not. This forces us to introduce a corrector function that removes the boundary inhomogeneity. While the construction of this function is not particularly delicate, the regularity required to do so dictates that we close our energy estimates at a higher order than we would otherwise. 
The paper proceeds as follows. The first half is devoted to the development of the linear theory and the proof of Theorem 1.1. In Section 2, we formulate a variational problem to find a growing-mode solution to the linearized Navier-Stokes-Poisson system. In Section 3, we show that our growing-mode solution grows at the largest possible rate. In the second half of the paper, we carry out our nonlinear analysis. In Section 4, we derive high-order nonlinear energy inequalities. Based on the linear growth and the nonlinear estimates, we then prove the bootstrap argument and Theorem 1.3 in Section 5.

\section{Construction of a growing mode solution to the linearized equations}

2A. Linearization around a stationary solution. We now linearize the equations in Lagrangian mass coordinates around the stationary solution $v=0, \rho=\rho_{0}, r=r_{0}$ (as defined by (1-23)). We will write $\sigma$ for the linearized density, and (by abuse of notation) $v$ for the linearized velocity. Then the linearized equations are given by

$$
\partial_{t} \sigma+4 \pi \rho_{0}^{2} \partial_{x}\left(r_{0}^{2} v\right)=0
$$

and

$$
\partial_{t} v+4 \pi r_{0}^{2} \partial_{x} \tilde{P}+\frac{x}{\pi r_{0}^{5}} \int_{0}^{x} \frac{\sigma(y, t)}{\rho_{0}^{2}(y)} d y=16 \pi^{2} r_{0}^{2} \partial_{x}\left(\left(\frac{4 \varepsilon}{3}+\delta\right) \rho_{0} \partial_{x}\left(r_{0}^{2} v\right)\right)
$$

where we have written $\tilde{P}=\gamma K \rho_{0}^{\gamma-1} \sigma$. The linearized boundary conditions are

$$
v(0, t)=0, \quad \sigma(M, t)=0
$$

and

$$
\tilde{P}-\frac{4 \varepsilon}{3}\left(4 \pi r_{0}^{2} \rho_{0} \partial_{x} v-\frac{v}{r_{0}}\right)-\delta\left(4 \pi r_{0}^{2} \rho_{0} \partial_{x} v+\frac{2 v}{r_{0}}\right)=0 \quad \text { at } x=M \quad \text { for all } t \geq 0
$$

Again, we can view (2-4) as a boundary condition only for $v$ since $\tilde{P}=\gamma K \rho_{0}^{\gamma-1} \sigma=0$ at $x=M$ for each $t \geq 0$.

It will often be useful for us to analyze a variant of this system, where we analyze the unknowns $\sigma$ and $w:=r_{0}^{2} v$. For these unknowns, Equations (2-1)-(2-4) become

$$
\begin{aligned}
& \partial_{t} \sigma+4 \pi \rho_{0}^{2} \partial_{x} w=0, \\
& \partial_{t} w+4 \pi r_{0}^{4} \partial_{x}\left(\gamma K \rho_{0}^{\gamma-1} \sigma\right)-4 r_{0} \partial_{x} P_{0} \int_{0}^{x} \frac{\sigma(y, t)}{\rho_{0}^{2}(y)} d y=16 \pi^{2} r_{0}^{4} \partial_{x}\left[\left(\frac{4 \varepsilon}{3}+\delta\right) \rho_{0} \partial_{x} w\right],
\end{aligned}
$$

along with the boundary conditions

$$
\frac{w}{r_{0}^{2}}(0, t)=\sigma(M, t)=0 \quad \text { and } \quad \frac{4 \varepsilon}{3}\left(4 \pi r_{0}^{3} \rho_{0} \partial_{x}\left(\frac{w}{r_{0}^{3}}\right)\right)+\delta\left(4 \pi \rho_{0} \partial_{x} w\right)=0 \quad \text { at } x=M .
$$

Note that $w / r_{0}^{2}=u$ is well-defined at $x=0$, but one may also view the first boundary condition in (2-6) in the sense of traces or limits. 
2B. Growing mode solution. We want to construct a growing mode solution to the linearized equations. We do so by looking for a solution of the form

$$
\sigma(x, t)=\sigma(x) e^{\lambda t} \quad \text { and } \quad v(x, t)=v(x) e^{\lambda t}
$$

for some $\lambda>0$. If we can find such a solution, then we say the solution is a growing mode since $\left|e^{\lambda t}\right| \rightarrow \infty$ as $t \rightarrow \infty$. Plugging the ansatz (2-7) into the linearized equations (2-1)-(2-4) and eliminating the time exponentials, we arrive at a pair of equations for $\sigma(x)$ and $v(x)$ :

$$
\lambda \sigma+4 \pi \rho_{0}^{2} \partial_{x}\left(r_{0}^{2} v\right)=0
$$

and

$$
\lambda v+4 \pi r_{0}^{2} \partial_{x} \tilde{P}+\frac{x}{\pi r_{0}^{5}} \int_{0}^{x} \frac{\sigma(y)}{\rho_{0}^{2}(y)} d y=16 \pi^{2} r_{0}^{2} \partial_{x}\left(\left(\frac{4 \varepsilon}{3}+\delta\right) \rho_{0} \partial_{x}\left(r_{0}^{2} v\right)\right),
$$

along with boundary conditions

$$
v(0)=\sigma(M)=0 \quad \text { and } \quad-\frac{4 \varepsilon}{3}\left(4 \pi r_{0}^{2} \rho_{0} \partial_{x} v-\frac{v}{r_{0}}\right)-\delta\left(4 \pi r_{0}^{2} \rho_{0} \partial_{x} v+\frac{2 v}{r_{0}}\right)=0 \quad \text { at } x=M .
$$

Our main result of this section establishes the existence of such a growing mode.

Theorem 2.1. There exist $\lambda>0$ and $\sigma, v:(0, M) \rightarrow \mathbb{R}$ that solve (2-8)-(2-10) and satisfy the following.

(1) $\sigma$ and $v$ are smooth on $(0, M)$ and satisfy (2-8)-(2-9) classically for $x \in(0, M)$.

(2) It holds that

$$
\limsup _{x \rightarrow 0} \frac{|v(x)|}{r_{0}(x)}+\limsup _{x \rightarrow 0}|\sigma(x)|+\limsup _{x \rightarrow 0}\left|\partial_{x}\left(r_{0}^{2} v\right)(x)\right|<\infty .
$$

In particular, $v(0)=0$.

(3) Let $\mathfrak{D}$ denote the linear operator $\mathfrak{D}=\rho_{0} \partial_{x}$. Then $\mathfrak{D}^{k} v$ and $\mathfrak{D}^{k}\left(\sigma / \rho_{0}\right)$ have well-defined traces at $x=M$ for every integer $k \geq 0$. In particular, $\sigma(M)=0$.

(4) $\lambda>0$ satisfies the variational characterization

$$
\begin{array}{r}
\lambda \int_{0}^{M}\left(\delta \rho_{0}\left|\partial_{x} \theta\right|^{2}+\frac{4 \varepsilon}{3} \rho_{0}\left|r_{0}^{3} \partial_{x}\left(\frac{\theta}{r_{0}^{3}}\right)\right|^{2}\right) d x+\int_{0}^{M}\left(\frac{\gamma P_{0} \rho_{0}}{2}\left|\partial_{x} \theta\right|^{2}+\frac{\partial_{x} P_{0}}{2 \pi r_{0}^{3}}|\theta|^{2}\right) d x \\
\geq-\lambda^{2} \int_{0}^{M} \frac{|\theta|^{2}}{16 \pi^{2} r_{0}^{4}} d x
\end{array}
$$

for every $\theta$ satisfying $\sqrt{\rho_{0}} \partial_{x} \theta \in L^{2}((0, M))$ and $\theta /\left(r_{0}^{2} \sqrt{\rho_{0}}\right) \in L^{2}((0, M))$. Note that for such $\theta$, it holds that $\theta /\left(r_{0}^{3} \sqrt{\rho_{0}}\right) \in L^{2}((0, M))$, which means that all of the integrals in (2-12) are well-defined.

(5) It holds that

$$
\int_{0}^{M}\left(\left|\frac{\sigma}{\rho_{0}}\right|^{2}+r_{0}^{2}\left|\partial_{x} \frac{\sigma}{\rho_{0}}\right|^{2}\right) d x+\int_{0}^{M}\left(\frac{\left|r_{0}^{2} v\right|^{2}}{r_{0}^{6} \rho_{0}}+\rho_{0}\left|\partial_{x}\left(r_{0}^{2} v\right)\right|^{2}+r_{0}^{2}\left|\partial_{x}\left(\rho_{0} \partial_{x}\left(r_{0}^{2} v\right)\right)\right|^{2}\right) d x<\infty .
$$


The proof of Theorem 2.1 will be completed in Section $2 \mathrm{~F}$. Throughout the rest of the section, we develop the tools needed in the proof. First we reformulate (2-8)-(2-10) to involve a single unknown function, $\phi$. The resulting problem for $\phi$ does not possess a standard variational structure since $\lambda$ appears both linearly and quadratically. To construct a solution using variational methods (required for proving (2-12), which is essential for the linear estimates of Section 3), we employ the technique of Guo and Tice [2010], which proceeds as follows. We modify the problem by replacing the linear appearance of $\lambda$ by an arbitrary parameter $s>0$. The resulting family (every $s>0$ ) of problems is amenable to solution by the constrained minimization of an energy functional, and for a range of $s$ we show that $\lambda=\lambda(s)>0$. We then study the behavior of $\lambda(s)$ as a function of $s$ and show that it is possible to find a unique fixed point such that $\lambda(s)=s>0$. This then yields the desired solution $\phi$, which in turn yields the solution to (2-8)-(2-10).

We begin by reducing to the study of a single unknown by introducing the function

$$
\phi(x):=\int_{0}^{x} \frac{\sigma(y)}{\rho_{0}^{2}(y)} d y .
$$

We may then use (2-8)-(2-10) to compute

$$
v=-\frac{\lambda}{4 \pi r_{0}^{2}} \phi, \quad \sigma=\rho_{0}^{2} \partial_{x} \phi, \quad \text { and } \quad \partial_{x} \tilde{P}=\partial_{x}\left(\gamma \rho_{0} P_{0} \partial_{x} \phi\right),
$$

where $P_{0}=K \rho_{0}^{\gamma}$. Using these and replacing in (2-9), we arrive at a second-order equation for $\phi$ :

$$
-\partial_{x}\left(\left(\frac{4 \lambda \varepsilon}{3}+\lambda \delta+\gamma P_{0}\right) \rho_{0} \partial_{x} \phi\right)+\frac{\partial_{x} P_{0}}{\pi r_{0}^{3}} \phi=-\frac{\lambda^{2}}{16 \pi^{2} r_{0}^{4}} \phi .
$$

The corresponding boundary conditions are

$$
\frac{\phi}{r_{0}^{2}}(0)=0 \quad \text { and } \quad \frac{4 \varepsilon}{3} \lambda\left(4 \pi r_{0}^{3} \rho_{0} \partial_{x}\left(\frac{\phi}{r_{0}^{3}}\right)\right)+\delta \lambda\left(4 \pi \rho_{0} \partial_{x} \phi\right)=0 \quad \text { at } x=M
$$

2C. Modification of the problem. Note that Theorem 2.1 is phrased in Lagrangian mass coordinates. This is because we will use these coordinates in our nonlinear analysis later in the paper. However, constructing the solution to (2-16)-(2-17) is somewhat easier if we make a change of variables back to the Eulerian radial coordinates associated to the stationary solution. To avoid confusion with the Eulerian radial coordinate for the nonlinear problem, we will call our new variable $z=r_{0}(x)$, where $r_{0}$ is given by (1-23). If $x \in(0, M)$ for $M$ the mass of the stationary star, then $z \in(0, R)$ for $R>0$ its radius. We will write $\varrho_{0}(z)=\rho_{0}(x)$ for the stationary density, $P_{0}=K \varrho^{\gamma}$, and $\varphi(z)=\phi(x)$ for the new unknown in $z$ coordinates. Then

$$
\partial_{x}=\frac{1}{4 \pi z^{2} \varrho_{0}} \partial_{z}
$$

In these coordinates, (2-16) becomes

$$
-\partial_{z}\left(\left(\frac{4 \lambda \varepsilon}{3}+\lambda \delta+\gamma P_{0}\right) \frac{\partial_{z} \varphi}{z^{2}}\right)+4 \frac{\partial_{z} P_{0}}{z^{3}} \varphi=-\frac{\lambda^{2} \varrho_{0}}{z^{2}} \varphi .
$$


For the boundary condition at $z=R$, we use (2-17) to see that

$$
\lambda \delta \frac{\partial_{z} \varphi(R)}{R^{2}}+\frac{4 \lambda \varepsilon}{3}\left(\frac{\partial_{z} \varphi(R)}{R^{2}}-3 \frac{\varphi(R)}{R^{3}}\right)=0 .
$$

At $z=0$ we enforce the boundary condition $\varphi(0)=0$. Once we have a solution in hand, we will show that, in fact, $\varphi(z) / z^{2} \rightarrow 0$ as $z \rightarrow 0$, which allows us to switch back to the boundary condition $\left(\phi / r_{0}^{2}\right)(0)=0$.

There is a difficulty in viewing (2-19)-(2-20) in a variational or Sturm-Liouville framework because of the appearance of $\lambda$ with two different homogeneities. To get around this issue, we temporarily modify the problem in order to restore the variational structure. Ultimately we will undo the modification and return to the proper formulation.

Fix $s>0$ and define

$$
\tilde{\varepsilon}=s \varepsilon \quad \text { and } \quad \tilde{\delta}=s \delta .
$$

Instead of (2-19), we will analyze the equation

$$
-\partial_{z}\left(\left(\frac{4 \tilde{\varepsilon}}{3}+\tilde{\delta}+\gamma P_{0}\right) \frac{\partial_{z} \varphi}{z^{2}}\right)+4 \frac{\partial_{z} P_{0}}{z^{3}} \varphi=-\frac{\lambda^{2} \varrho_{0}}{z^{2}} \varphi
$$

for arbitrary $s>0$. We couple this equation to the boundary conditions $\varphi(0)=0$ and

$$
\tilde{\delta} \frac{\partial_{z} \varphi(R)}{R^{2}}+\frac{4 \tilde{\varepsilon}}{3}\left(\frac{\partial_{z} \varphi(R)}{R^{2}}-3 \frac{\varphi(R)}{R^{3}}\right)=0 .
$$

Modifying the problem in this way restores the variational structure. Indeed, in (2-22) the $\lambda^{2}$ term can be viewed as an eigenvalue. Thinking of the principal eigenvalue $\lambda$ as a function of $s$, that is, $\lambda=\lambda(s)$, we will show that it is possible to choose $s$ such that $\lambda(s)>0$ and $s=\lambda(s)$, which returns us to the original problem and yields a growing-mode solution.

2D. Constrained minimization. In order to construct solutions to (2-22)-(2-23), we will employ a constrained minimization. To begin, we define the function space on which the energy functionals will be defined. For $\tau>0$, we define the weighted Sobolev space $H_{\tau}^{1}((0, R))$ as the completion of $\left\{u \in C^{\infty}([0, R]) \mid u(0)=0\right\}$ with respect to the norm

$$
\|u\|_{H_{\tau}^{1}}^{2}=\int_{0}^{R} \frac{\left|u^{\prime}(z)\right|^{2}+|u(z)|^{2}}{z^{\tau}} d z,
$$

where $^{\prime}=d / d z$. This weighted Sobolev space possesses the same sort of embedding (continuous and compact) properties as the usual space $H^{1}$. Since these results are not widely available in the literature, we record them in the following lemma.

Lemma 2.2. (1) For $u \in H_{\tau}^{1}((0, R))$, we have the inequalities

$$
\sup _{0 \leq z \leq R}\left|u(z) z^{-(\tau+1) / 2}\right| \leq \frac{1}{\sqrt{1+\tau}}\left(\int_{0}^{R} \frac{\left|u^{\prime}(z)\right|^{2}}{z^{\tau}} d z\right)^{1 / 2}
$$

and

$$
\int_{0}^{R} \frac{|u(z)|^{2}}{z^{\tau+2}} d z \leq \frac{4}{(1+\tau)^{2}} \int_{0}^{R} \frac{\left|u^{\prime}(z)\right|^{2}}{z^{\tau}} d z .
$$


(2) Let $0 \leq \alpha<1$. We have the compact embedding $H_{\tau}^{1}((0, R)) \Subset L_{\tau+1+\alpha}^{2}((0, R))$, where the latter space is the weighted $L^{2}$ space with norm

$$
\|u\|_{L_{\tau+1+\alpha}^{2}}^{2}=\int_{0}^{R} \frac{|u(z)|^{2}}{z^{\tau+1+\alpha}} d z
$$

Proof. We begin with the inequalities in item (1). By approximation, we may assume that $u$ is smooth and $u(0)=0$. Then

$$
\begin{aligned}
|u(z)|=|u(z)-u(0)| \leq \int_{0}^{z}\left|u^{\prime}(t)\right| d t & \leq\left(\int_{0}^{z} t^{\tau} d t\right)^{1 / 2}\left(\int_{0}^{z} \frac{\left|u^{\prime}(t)\right|^{2}}{t^{\tau}} d t\right)^{1 / 2} \\
& \leq\left(\frac{z^{\tau+1}}{\tau+1}\right)^{1 / 2}\left(\int_{0}^{R} \frac{\left|u^{\prime}(t)\right|^{2}}{t^{\tau}} d t\right)^{1 / 2},
\end{aligned}
$$

which yields the first inequality. To get the second, we recall an inequality due to G. H. Hardy:

$$
\left(\int_{0}^{\infty}\left(\int_{0}^{z}|f(t)| d t\right)^{p} \frac{d z}{z^{b+1}}\right)^{1 / p} \leq \frac{p}{b}\left(\int_{0}^{\infty}|f(z)|^{p} z^{p-b-1} d z\right)^{1 / p}
$$

for $1 \leq p<\infty$ and $0<b<\infty$, which follows immediately from Young's inequality on the multiplicative group $(0, \infty)$ with measure $d t / t$ by convolving $|f(t)| t^{1-b / p}$ with $t^{-b / p} \chi_{(1, \infty)}(t)$. Then $|u(z)| \leq \int_{0}^{z}\left|u^{\prime}(t)\right| d t$ implies that

$$
\int_{0}^{R} \frac{|u(z)|^{2}}{z^{\tau+2}} d z \leq \int_{0}^{R}\left(\int_{0}^{z}\left|u^{\prime}(t)\right| d t\right)^{2} \frac{d z}{z^{\tau+2}} .
$$

Applying Hardy's inequality to the right side with $f=u^{\prime} \chi_{(0, R)}, b=\tau+1$, and $p=2$ yields

$$
\int_{0}^{R} \frac{|u(z)|^{2}}{z^{\tau+2}} d z \leq \frac{4}{(\tau+1)^{2}} \int_{0}^{R} \frac{\left|u^{\prime}(z)\right|^{2}}{z^{\tau}} d z
$$

which is the desired inequality.

We now prove the compactness result. Assume that $\left\|u_{n}\right\|_{H_{\tau}^{1}} \leq C$ for $n \in \mathbb{N}$. Fix $\kappa>0$. We claim that there exists a subsequence $\left\{u_{n_{i}}\right\}$ such that

$$
\sup _{i, j}\left\|u_{n_{i}}-u_{n_{j}}\right\|_{L_{\tau+1+\alpha}^{2}} \leq \kappa .
$$

To prove the claim, let $z_{0} \in(0, R)$ be chosen such that

$$
z_{0}^{1-\alpha} \frac{C^{2}}{(1+\tau)(1-\alpha)} \leq \frac{\kappa}{2} .
$$

Then since the subinterval $\left(z_{0}, R\right)$ avoids the singularity of $1 / z^{\tau},\left.u_{n}\right|_{\left(z_{0}, R\right)}$ is uniformly bounded in $H^{1}\left(\left(z_{0}, R\right)\right)$. By the compact embedding $H^{1}\left(\left(z_{0}, R\right)\right) \Subset C^{0}\left(\left(z_{0}, R\right)\right)$, we may extract a subsequence $\left\{u_{n_{i}}\right\}$ that converges in $L^{\infty}\left(\left(z_{0}, R\right)\right)$. We are free to restrict the subsequence to large enough values of $i$ that

$$
\left\|u_{n_{i}}-u_{n_{j}}\right\|_{L^{\infty}\left(\left(z_{0}, R\right)\right)}^{2} \leq \frac{\kappa z_{0}^{\tau+1+\alpha}}{2\left(R-z_{0}\right)} \quad \text { for all } i, j .
$$


Then along this subsequence we can apply the first inequality in item (1) to get

$$
\begin{aligned}
\int_{0}^{R} \frac{\left|u_{n_{i}}(z)-u_{n_{j}}(z)\right|^{2}}{z^{\tau+1+\alpha}} d z & =\int_{0}^{z_{0}} \frac{\left|u_{n_{i}}(z)-u_{n_{j}}(z)\right|^{2}}{z^{\tau+1+\alpha}} d z+\int_{z_{0}}^{R} \frac{\left|u_{n_{i}}(z)-u_{n_{j}}(z)\right|^{2}}{z^{\tau+1+\alpha}} d z \\
& \leq \frac{C^{2}}{1+\tau} \int_{0}^{z_{0}} \frac{d z}{z^{\alpha}}+\frac{R-z_{0}}{z_{0}^{\tau+1+\alpha}}\left\|u_{n_{i}}-u_{n_{j}}\right\|_{L^{\infty}\left(\left(z_{0}, R\right)\right)}^{2} \leq \kappa,
\end{aligned}
$$

which proves the claim. Now we may use the claim with $\kappa=1 / k, k \in \mathbb{N}$ and employ a standard diagonal argument to extract a subsequence converging in $L_{\tau+1+\alpha}^{2}((0, R))$.

Remark 2.3. The inequality (2-26) implies that we can take the norm on $H_{\tau}^{1}$ to be

$$
\|u\|_{H_{\tau}^{1}}^{2}=\int_{0}^{R} \frac{\left|u^{\prime}(z)\right|^{2}}{z^{\tau}} d z
$$

We can now define the energy functionals to use in the constrained minimization. Let

$$
E(\varphi)=\int_{0}^{R}\left[\left(\tilde{\delta}+\gamma P_{0}\right) \frac{\left|\partial_{z} \varphi\right|^{2}}{z^{2}}+\frac{4 \tilde{\varepsilon}}{3 z^{2}}\left|\partial_{z} \varphi-3 \frac{\varphi}{z}\right|^{2}+4 \frac{\partial_{z} P_{0}}{z^{3}}|\varphi|^{2}\right] d z
$$

and

$$
J(\varphi)=\int_{0}^{R} \frac{\varrho_{0}}{z^{2}}|\varphi|^{2} d z
$$

By (2-26) in Lemma 2.2, both $E$ and $J$ are well-defined on the space $H_{2}^{1}((0, R))$. Note, though, that $E$ is not positive definite since $\partial_{z} P_{0}<0$. Define the set

$$
\mathscr{A}:=\left\{\varphi \in H_{2}^{1}((0, R)) \mid J(\varphi)=1\right\} .
$$

We will build solutions to (2-22) by minimizing $E$ over $\mathscr{A}$. First we show that such a minimizer exists.

Proposition 2.4. E achieves its infimum on the set $A$.

Proof. To begin, we show that $E$ is coercive on $\mathscr{A}$, which amounts to controlling the last term in $E$. Recall that by $(1-9), \varrho_{0}(z) \sim(R-z)^{1 /(\gamma-1)}$ for $z$ near $R$. This implies that

$$
\frac{\partial_{z} P_{0}}{\varrho_{0}}=\gamma K \varrho_{0}^{\gamma-2} \partial_{z} \varrho_{0}=\frac{\gamma K}{\gamma-1} \partial_{z}\left(\varrho_{0}^{\gamma-1}\right)
$$

is bounded near $z=R$. Since $\varrho_{0}$ and $P_{0}=K \varrho_{0}^{\gamma}$ are smooth and bounded below away from $z=R$, this implies that

$$
\left\|\frac{\partial_{z} P_{0}}{\varrho_{0}}\right\|_{L^{\infty}((0, R))}<\infty
$$

Then for any $z_{0} \in(0, R)$, we have the bound 


$$
\begin{aligned}
\int_{0}^{R}\left|\partial_{z} P_{0}\right| \frac{|\varphi|^{2}}{z^{3}} d z & =\int_{0}^{z_{0}}\left|\partial_{z} P_{0}\right| \frac{z|\varphi|^{2}}{z^{4}} d z+\int_{z_{0}}^{R} \frac{\left|\partial_{z} P_{0}\right|}{\varrho_{0}} \frac{\varrho_{0}|\varphi|^{2}}{z^{2}} d z \\
& \leq z_{0}\left\|\partial_{z} P_{0}\right\|_{L^{\infty}} \int_{0}^{z_{0}} \frac{|\varphi|^{2}}{z^{4}} d z+\frac{1}{z_{0}}\left\|\frac{\partial_{z} P_{0}}{\varrho_{0}}\right\|_{L^{\infty}} \int_{z_{0}}^{R} \frac{\varrho_{0}|\varphi|^{2}}{z^{2}} d z \\
& \leq z_{0} \frac{4}{9}\left\|\partial_{z} P_{0}\right\|_{L^{\infty}} \int_{0}^{R} \frac{\left|\partial_{z} \varphi\right|^{2}}{z^{2}} d z+\frac{1}{z_{0}}\left\|\frac{\partial_{z} P_{0}}{\varrho_{0}}\right\|_{L^{\infty}} .
\end{aligned}
$$

For the second inequality we have used Lemma 2.2 and the fact that $\varphi \in \mathscr{A}$. Then by choosing $z_{0}$ sufficiently small, we have that

$$
E(\varphi) \geq-C_{z_{0}}+\int_{0}^{R}\left[\left(\frac{\tilde{\delta}}{2}+\gamma P_{0}\right) \frac{\left|\partial_{z} \varphi\right|^{2}}{z^{2}}+\frac{4 \tilde{\varepsilon}}{3 z^{2}}\left|\partial_{z} \varphi-3 \frac{\varphi}{z}\right|^{2}\right] d z
$$

for a constant $C_{z_{0}}>0$ depending on the choice of $z_{0}$, which immediately yields the desired coercivity since $\tilde{\delta}>0$.

With the coercivity in hand, we may deduce the existence of a minimizer by using the standard direct methods, employing Lemma 2.2 for compactness.

Since a minimizer exists, we can now define the function $\mu:(0, \infty) \rightarrow \mathbb{R}$ by

$$
\mu(s)=\inf _{\varphi \in \mathscr{A}} E(\varphi ; s),
$$

where we write $E(\varphi)=E(\varphi ; s)$ to emphasize the dependence of $E$ on the parameter $s>0$, that is,

$$
E(\varphi ; s)=s \int_{0}^{R}\left[\delta \frac{\left|\partial_{z} \varphi\right|^{2}}{z^{2}}+\frac{4 \varepsilon}{3 z^{2}}\left|\partial_{z} \varphi-3 \frac{\varphi}{z}\right|^{2}\right] d z+\int_{0}^{R}\left[\gamma P_{0} \frac{\left|\partial_{z} \varphi\right|^{2}}{z^{2}}+4 \frac{\partial_{z} P_{0}}{z^{3}}|\varphi|^{2}\right] d z .
$$

The minimizer we have constructed satisfies Euler-Lagrange equations of the form (2-22).

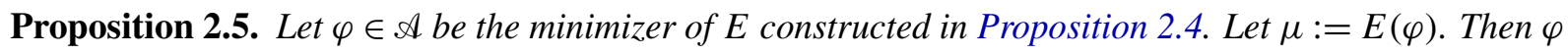
is smooth on $(0, R]$ and satisfies

$$
-\partial_{z}\left(\left(\frac{4 \tilde{\varepsilon}}{3}+\tilde{\delta}+\gamma P_{0}\right) \frac{\partial_{z} \varphi}{z^{2}}\right)+4 \frac{\partial_{z} P_{0}}{z^{3}} \varphi=\frac{\mu \varrho_{0}}{z^{2}} \varphi
$$

along with the boundary conditions $\varphi(0)=0$ and

$$
\tilde{\delta} \frac{\partial_{z} \varphi(R)}{R^{2}}+\frac{4 \tilde{\varepsilon}}{3}\left(\frac{\partial_{z} \varphi(R)}{R^{2}}-3 \frac{\varphi(R)}{R^{3}}\right)=0 .
$$

Proof. Fix $\varphi_{0} \in H_{2}^{1}((0, R))$. Define

$$
j(t, \tau)=J\left(\varphi+t \varphi_{0}+\tau \varphi\right)
$$

and note that $j(0,0)=1$. Moreover, $j$ is smooth and

$$
\frac{\partial j}{\partial t}(0,0)=2 \int_{0}^{R} \varrho_{0} \frac{\varphi_{0} \varphi}{z^{2}} d z \quad \text { and } \quad \frac{\partial j}{\partial \tau}(0,0)=2 \int_{0}^{R} \varrho_{0} \frac{\varphi^{2}}{z^{2}} d z=2 .
$$


So, by the inverse function theorem, we can solve for $\tau=\tau(t)$ in a neighborhood of 0 as a $C^{1}$ function of $t$ such that $\tau(0)=0$ and $j(t, \tau(t))=1$. We may differentiate the last equation to find

$$
\frac{\partial j}{\partial t}(0,0)+\frac{\partial j}{\partial \tau}(0,0) \tau^{\prime}(0)=0
$$

and hence

$$
\tau^{\prime}(0)=-\frac{1}{2} \frac{\partial j}{\partial t}(0,0)=-\int_{0}^{R} \varrho_{0} \frac{\varphi_{0} \varphi}{z^{2}} d z
$$

Since $\varphi$ is a minimizer over $\mathcal{A}$, we then have

$$
0=\left.\frac{d}{d t}\right|_{t=0} E\left(\varphi+t \varphi_{0}+\tau(t) \varphi\right)
$$

which implies that

$$
\begin{aligned}
0=\int_{0}^{R} \frac{\tilde{\delta}+\gamma P_{0}}{z^{2}} \partial_{z} \varphi\left(\partial_{z} \varphi_{0}+\tau^{\prime}(0) \partial_{z} \varphi\right) d z & +\int_{0}^{R} 4 \frac{\partial_{z} P_{0}}{z^{3}} \varphi\left(\varphi_{0}+\tau^{\prime}(0) \varphi\right) d z \\
& +\int_{0}^{R} \frac{4 \tilde{\varepsilon}}{3 z^{2}}\left(\partial_{z} \varphi-3 \frac{\varphi}{z}\right)\left(\partial_{z} \varphi_{0}-3 \frac{\varphi_{0}}{z}+\tau^{\prime}(0)\left(\partial_{z} \varphi-3 \frac{\varphi}{z}\right)\right) d z .
\end{aligned}
$$

Rearranging and plugging in the value of $\tau^{\prime}(0)$, we may rewrite this equation as $\mu \int_{0}^{R} \frac{\varrho_{0}}{z^{2}} \varphi_{0} \varphi d z$

$$
=\int_{0}^{R} \frac{\tilde{\delta}+\gamma P_{0}}{z^{2}} \partial_{z} \varphi \partial_{z} \varphi_{0} d z+\int_{0}^{R} 4 \frac{\partial_{z} P_{0}}{z^{3}} \varphi \varphi_{0} d z+\int_{0}^{R} \frac{4 \tilde{\varepsilon}}{3 z^{2}}\left(\partial_{z} \varphi-3 \frac{\varphi}{z}\right)\left(\partial_{z} \varphi_{0}-3 \frac{\varphi_{0}}{z}\right) d z
$$

where the eigenvalue is $\mu=E(\varphi)$.

By making variations with $\varphi_{0}$ compactly supported in $(0, R)$, we find that $\varphi$ satisfies (2-46) in a weak sense in $(0, R)$. Standard bootstrapping arguments then show that $\varphi \in H^{k}\left(\left(z_{0}, R\right)\right)$ for all $k \geq 0$ and $0<z_{0}<R$, and hence $\varphi$ is smooth in $(0, R]$. This implies that the equations are also classically satisfied. Since $\varphi \in H^{2}((R / 2, R))$, the traces of $\varphi, \partial_{z} \varphi$ are well-defined at the endpoint $z=R$. Making variations with respect to arbitrary $\varphi_{0} \in C_{c}^{\infty}((0, R])$, we find that the boundary condition (2-47) is satisfied. The condition $\varphi(0)=0$ is satisfied by virtue of Lemma 2.2.

We now want to show that the minimizers, which are solutions to (2-46), satisfy the asymptotic condition $|\varphi(z)| / z^{2} \rightarrow 0$ as $z \rightarrow 0$. As a preliminary step, we record an asymptotic result for solutions to a more generic ODE.

Lemma 2.6 [Lin 1997, Proposition A.1]. Suppose that $\psi(\tau)$ solves

$$
\psi^{\prime \prime}(\tau)+\left(\alpha \tau^{-1}+g(\tau)\right) \psi^{\prime}(\tau)+\tau^{-1} f(\tau) \psi(\tau)=0 \quad \text { if } 0<\tau<\tau_{0},
$$

where $^{\prime}=d / d \tau$ and $f, g \in C^{0}\left(\left[0, \tau_{0}\right]\right)$. If $\alpha<0$, then either $\psi(0) \neq 0$ or

$$
|\psi(\tau)| \leq \frac{C}{\tau^{\alpha-1}} \quad \text { and } \quad\left|\psi^{\prime}(\tau)\right| \leq \frac{C}{\tau^{\alpha}}
$$


Proof. The case $\alpha>2$ is the content of Proposition A.1 of [Lin 1997], but the proof of the proposition also shows the result when $\alpha<0$.

Next we use this lemma to establish the asymptotics at $z=0$ for solutions to (2-46).

Lemma 2.7. Suppose $\varphi$ is a solution of (2-46). Then $|\varphi(z)| \leq C z^{3}$ and $\left|\varphi^{\prime}(z)\right| \leq C z^{2}$ near $z=0$.

Proof. We begin by rewriting (2-46). Define $X=\tilde{\varepsilon}+\tilde{\delta}+\gamma P_{0}=\tilde{\varepsilon}+\tilde{\delta}+\gamma K \varrho_{0}^{\gamma}$ and $X_{0}=\tilde{\varepsilon}+P_{0}=\tilde{\varepsilon}+K \varrho_{0}^{\gamma}$. Then (2-46) is equivalent to the equation

$$
\varphi^{\prime \prime}+\left(\frac{X^{\prime}}{X}-\frac{2}{z}\right) \varphi^{\prime}-4 \frac{X_{0}^{\prime}}{z X} \varphi=-\mu \frac{\varrho_{0}}{X} \varphi .
$$

Note that $X^{\prime} / X, X_{0}^{\prime} / X$, and $\varrho_{0} / X$ are all continuous at $z=0$, so we may apply Lemma 2.6 with $\alpha=-2$ to deduce that either $\varphi(0) \neq 0$ or $|\varphi(z)| \leq C z^{3}$ and $\left|\varphi^{\prime}(z)\right| \leq C z^{2}$ near $z=0$. By Lemma 2.2, the former condition cannot hold, so the latter conditions must be the case.

2E. Properties of the eigenvalue $\mu(s)$. It is convenient to decompose $E$ according to

$$
E(\varphi ; s)=E_{0}(\varphi)+s E_{1}(\varphi)
$$

for

$$
E_{0}(\varphi):=\int_{0}^{R}\left(\gamma P_{0} \frac{\left|\partial_{z} \varphi\right|^{2}}{z^{2}}+4 \frac{\partial_{z} P_{0}}{z^{3}}|\varphi|^{2}\right) d z
$$

and

$$
E_{1}(\varphi):=\int_{0}^{R}\left(\delta \frac{\left|\partial_{z} \varphi\right|^{2}}{z^{2}}+\frac{4 \varepsilon}{3 z^{2}}\left|\partial_{z} \varphi-3 \frac{\varphi}{z}\right|^{2}\right) d z \geq 0 .
$$

Notice that since $E_{0}$ does not involve either $\delta$ or $\varepsilon$, we may view it as the "inviscid" part of $E$. Because $\partial_{z} P_{0}$ lacks a sign, $E_{0}$ fails to be nonnegative. However, an easy modification of the argument used in Proposition 2.4 shows that $\inf _{\mathscr{A}} E_{0}>-\infty$. As a consequence of the analysis of the inviscid problem, carried out by Lin [1997], we have that this infimum is actually negative and is achieved, and its value characterizes the fastest growing mode for the inviscid problem. Indeed, there exists a $\varphi_{0} \in \mathscr{A}$ such that

$$
0>-\chi_{0}^{2}:=\inf _{\varphi \in \mathscr{A}} E_{0}(\varphi)=E_{0}\left(\varphi_{0}\right),
$$

with $\chi_{0}>0$ the fastest growth rate and $\varphi_{0}$ the corresponding growing mode solution for the linearized inviscid problem.

We are ultimately concerned with finding $\mu=-\lambda^{2}$ for some $\lambda>0$. This requires us to work in a range of $s$ such that $\mu(s)<0$. Our next result shows that $\mu(s)<0$ for $s$ sufficiently small.

Lemma 2.8. There exist constants $C_{1}, C_{2} \geq 0$ depending on $\varphi_{0}$ such that

$$
\mu(s) \leq s\left(\delta C_{1}+\varepsilon C_{2}\right)-\chi_{0}^{2} .
$$

In particular, $\mu(s)<0$ for $s$ sufficiently small. 
Proof. Let $\varphi_{0} \in \mathscr{A}$ be the minimizer of $E_{0}$ from (2-61). Then, using the decomposition (2-58), we find

$$
\mu(s)=\inf _{\mathscr{A}} E \leq E\left(\varphi_{0}\right)=E_{0}\left(\varphi_{0}\right)+s E_{1}\left(\varphi_{0}\right)=s E_{1}\left(\varphi_{0}\right)-\chi_{0}^{2} .
$$

Then $E_{1}\left(\varphi_{0}\right)=\delta C_{1}+\varepsilon C_{2}$, where

$$
C_{1}=\int_{0}^{R} \frac{\left|\partial_{z} \varphi_{0}\right|^{2}}{z^{2}} d z>0 \quad \text { and } \quad C_{2}=\int_{0}^{R} \frac{4}{3 z^{2}}\left|\partial_{z} \varphi_{0}-3 \frac{\varphi_{0}}{z}\right|^{2} d z>0 .
$$

Strict inequality holds for $C_{1}$ since $\varphi_{0} \in \mathcal{A}$, while it holds for $C_{2}$ since $C_{2}=0$ if and only if $\varphi_{0}(z)=\alpha z^{3}$ for some $\alpha \in \mathbb{R}$, but one can check that this is not a solution to (2-46) with $\tilde{\varepsilon}, \tilde{\delta}=0$.

The next proposition proves some crucial monotonicity and continuity properties of $\mu(s)$ for $s>0$.

Proposition 2.9. (1) $\mu(s)$ is strictly increasing in $s$.

(2) There exists a constant $C_{3}>0$ such that

$$
\mu(s) \geq-\chi_{0}^{2}+s C_{3} \delta
$$

where $\chi_{0}>0$ is given in (2-61).

(3) $\mu$ is locally Lipschitz on $(0, \infty)$, and in particular, $\mu$ is continuous on $(0, \infty)$.

Proof. We begin by establishing some notation. According to Proposition 2.4 , for each $s \in(0, \infty)$ we can find $\varphi_{s} \in \mathscr{A}$ such that

$$
E\left(\varphi_{s} ; s\right)=\inf _{\varphi \in \mathscr{A}} E(\varphi ; s)=\mu(s)
$$

Next, we recall the decomposition of $E$ given in (2-58) and note that the nonnegativity of $E_{1}$ implies that $E$ is nondecreasing in $s$ with $\varphi \in \mathscr{A}$ kept fixed.

To prove the first assertion, note that if $s_{1}, s_{2} \in(0, \infty)$ with $s_{1} \leq s_{2}$, then the minimality of $\varphi_{s_{i}}$ and the nonnegativity of $E_{1}$ imply that

$$
\mu\left(s_{1}\right)=E\left(\varphi_{s_{1}} ; s_{1}\right) \leq E\left(\varphi_{s_{2}} ; s_{1}\right) \leq E\left(\varphi_{s_{2}} ; s_{2}\right)=\mu\left(s_{2}\right) .
$$

This shows that $\mu$ is nondecreasing in $s$. Suppose by way of contradiction that $\mu\left(s_{1}\right)=\mu\left(s_{2}\right)$ for $s_{1} \neq s_{2}$. Then the last inequality implies that

$$
s_{1} E_{1}\left(\varphi_{s_{2}}\right)=s_{2} E_{1}\left(\varphi_{s_{2}}\right)
$$

which means that $E_{1}\left(\varphi_{s_{2}}\right)=0$. The vanishing of $E_{1}\left(\varphi_{s_{2}}\right)$ implies that $\varphi_{s_{2}}=0$, which is impossible since $\varphi_{s_{2}} \in \mathcal{A}$. Hence equality cannot be achieved, and $\mu$ is strictly increasing in $s$.

Now note that (2-58), the nonnegativity of $E_{1}$, and (2-61) imply that

$$
\mu(s) \geq \inf _{\varphi \in \mathscr{A}} E_{0}(\varphi)+s \inf _{\varphi \in \mathscr{A}} E_{1}(\varphi)=-\chi_{0}^{2}+s \inf _{\varphi \in \mathscr{A}} E_{1}(\varphi) .
$$

It is a simple matter to see that

$$
\inf _{\varphi \in \mathscr{A}} E_{1}(\varphi) \geq \delta \inf _{\varphi \in \mathscr{A}} \int_{0}^{R} \frac{\left|\partial_{z} \varphi\right|^{2}}{z^{2}} d z:=C_{3} \delta>0 .
$$

The second assertion follows. 
Now fix $Q=[a, b] \Subset(0, \infty)$, and fix any $\psi \in \mathscr{A}$. Again by the nonnegativity of $E_{1}$ and the minimality of $\varphi_{s}$, we deduce that

$$
E(\psi ; b) \geq E(\psi ; s) \geq E\left(\varphi_{s} ; s\right) \geq a E_{1}\left(\varphi_{s}\right)-\chi_{0}^{2}
$$

for all $s \in Q$. This implies that there exists a constant $0<C=C(a, b, \psi, \gamma, K)<\infty$ such that

$$
\sup _{s \in Q} E_{1}\left(\varphi_{s}\right) \leq C .
$$

Let $s_{1}, s_{2} \in Q$. Using the minimality of $\varphi_{s_{1}}$ compared to $\varphi_{s_{2}}$, we know that

$$
\mu\left(s_{1}\right)=E\left(\varphi_{s_{1}} ; s_{1}\right) \leq E\left(\varphi_{s_{2}} ; s_{1}\right),
$$

but from our decomposition (2-58), we may bound

$$
E\left(\varphi_{s_{2}} ; s_{1}\right) \leq E\left(\varphi_{s_{2}} ; s_{2}\right)+\left|s_{1}-s_{2}\right| E_{1}\left(\varphi_{s_{2}}\right)=\mu\left(s_{2}\right)+\left|s_{1}-s_{2}\right| E_{1}\left(\varphi_{s_{2}}\right) .
$$

Chaining these two inequalities together and employing (2-72), we find that

$$
\mu\left(s_{1}\right) \leq \mu\left(s_{2}\right)+C\left|s_{1}-s_{2}\right| .
$$

Reversing the role of the indices 1 and 2 in the derivation of this inequality gives the same bound with $s_{1}$ switched with $s_{2}$. We deduce that

$$
\left|\mu\left(s_{1}\right)-\mu\left(s_{2}\right)\right| \leq C\left|s_{1}-s_{2}\right|,
$$

which proves item (3).

Now we know that the eigenvalue $\mu(s)$ is negative as long as $s<\frac{\chi_{0}^{2}}{\delta C_{1}+\varepsilon C_{2}}$ and that $\mu$ is continuous on $(0, \infty)$. We can then define the nonempty open set

$$
\Omega=\mu^{-1}((-\infty, 0)) \subset(0, \infty),
$$

on which we can calculate $\lambda(s)=\sqrt{-\mu(s)}>0$.

It turns out that the set $\Omega$ is sufficiently large to find $s>0$ such that $\lambda(s)=s$. This inversion will then allow us to solve the original growing-mode equations.

Proposition 2.10. There exists a unique $s \in \Omega$ such that $\lambda(s)=\sqrt{-\mu(s)}>0$ and $\lambda(s)=s$.

Proof. According to Lemma 2.8, we know that $\mu(s)<0$ for $s \in\left[0, \chi_{0}^{2} /\left(\delta C_{1}+\varepsilon C_{2}\right)\right)$. Moreover, the lower bound (2-65) in Proposition 2.9 implies that $\mu(s) \rightarrow+\infty$ as $s \rightarrow \infty$. This implies the existence of $s_{0} \in(0, \infty)$ such that $\Omega=\left(0, s_{0}\right)$, which means that $\lambda\left(s_{0}\right)=0$. Define the function $\Psi:\left(0, s_{0}\right) \rightarrow(0, \infty)$ by $\Psi(s)=s / \lambda(s)$. The monotonicity and continuity properties of $\mu$ are inherited by $\Psi$, that is, $\Psi$ is continuous on $\left(0, s_{0}\right)$ and strictly increases from 0 to $+\infty$ as $s \rightarrow s_{0}$. As such, we may apply the intermediate value theorem to find a unique $s \in\left(0, s_{0}\right)$ such that $\Psi(s)=1$. For this $s$, we then have that $s=\lambda(s)$, the desired result.

Up to now we have viewed the viscosity parameters $\varepsilon, \delta$ as being fixed. With the unique fixed point $\lambda(s)=\sqrt{-\mu(s)}=s>0$ in hand, we can now consider the behavior of $\lambda$ with respect to the viscosity parameters, $\varepsilon, \delta$. To this end, let us write $\lambda=\lambda(\delta, \varepsilon)$ in the following. 
Proposition 2.11. Write $\lambda=\lambda(\delta, \varepsilon)>0$ for the unique $\lambda$ produced by Proposition 2.10 for a given $\varepsilon, \delta>0$. Then

$$
\lim _{(\delta, \varepsilon) \rightarrow 0} \lambda(\delta, \varepsilon)=\chi_{0}
$$

and

$$
\lim _{\delta \rightarrow \infty} \lambda(\delta, \varepsilon)=0 .
$$

Proof. Combining the estimate from Lemma 2.8 with (2-65) from Proposition 2.9 and employing Proposition 2.10, we find that

$$
\lambda(\delta, \varepsilon)^{2}+\delta C_{3} \lambda(\delta, \varepsilon)-\chi_{0}^{2} \leq 0 \leq \lambda(\delta, \varepsilon)^{2}+\left(\delta C_{1}+\varepsilon C_{2}\right) \lambda(\delta, \varepsilon)-\chi_{0}^{2},
$$

for constants $C_{1}, C_{2}, C_{3}>0$ independent of $\varepsilon, \delta$. The first inequality in (2-80) implies that

$$
\lambda(\delta, \varepsilon) \leq \frac{1}{2}\left(-\delta C_{3}+\sqrt{\delta^{2} C_{3}^{2}+4 \chi_{0}^{2}}\right),
$$

while the second and the fact that $\lambda(\delta, \varepsilon)>0$ imply that

$$
\lambda(\delta, \varepsilon) \geq \frac{1}{2}\left(-\left(\delta C_{1}+\varepsilon C_{2}\right)+\sqrt{\left(\delta C_{1}+\varepsilon C_{2}\right)^{2}+4 \chi_{0}^{2}}\right) .
$$

Sending $(\delta, \varepsilon) \rightarrow 0$ and chaining together (2-81) and (2-82) then yields (2-78). On the other hand, expanding the right side of (2-81) for large $\delta$ shows that

$$
0 \leq \lambda(\delta, \varepsilon) \leq \frac{\chi_{0}^{2}}{\delta C_{3}}+o(1), \quad \text { for } o(1) \rightarrow 0 \quad \text { as } \delta \rightarrow \infty
$$

which implies (2-79).

Remark 2.12. Proposition 2.11 has two important consequences. The first is that the fastest inviscid growth rate is recovered in the inviscid limit $(\delta, \varepsilon) \rightarrow 0$. This can be understood as a continuity result. The second is that large bulk viscosity suppresses the viscous growth rate, and for sufficiently large $\delta$, the growth rate is very slow. This causes the delay of the instability occurrence time.

2F. Proof of Theorem 2.1. We now combine our above analysis to deduce the existence of a solution $\varphi$, $\lambda>0$ to $(2-19)-(2-20)$.

Theorem 2.13. There exist $\lambda>0$ and $\varphi \in H_{2}^{1}((0, R))$, smooth on $(0, R]$, that solve (2-19) along with the boundary condition (2-20). The solution satisfies the asymptotics $|\varphi(z)| \leq C z^{3}$ and $\left|\partial_{z} \varphi(z)\right| \leq C z^{2}$ as $z \rightarrow 0$.

Proof. Combining Propositions 2.5 and 2.10, we see that there exists a solution to (2-22) and (2-23) for $\lambda(s)=\sqrt{-\mu(s)}>0$, satisfying $s=\lambda(s)$. This implies that the solution is actually a solution to (2-19) and (2-20). The asymptotics at $z=0$ follow from Lemma 2.7 .

An immediate consequence of Theorem 2.13 is the existence of a solution to (2-16)-(2-17). 
Corollary 2.14. There exist $\lambda>0$ and $\phi(x)=\varphi\left(r_{0}(x)\right)$, smooth on $(0, M)$, that solve (2-16)-(2-17). The solution satisfies

$$
\limsup _{x \rightarrow 0} \frac{|\phi(x)|}{r_{0}^{3}(x)}+\limsup _{x \rightarrow 0}\left|\partial_{x} \phi(x)\right|<\infty .
$$

Let $\mathfrak{D}$ denote the linear operator $\mathfrak{D} \phi(x)=\rho_{0}(x) \partial_{x} \phi(x)$. The solution satisfies the property that $\mathfrak{D}^{k} \phi$ has a well-defined trace at $x=M$ for every integer $k \geq 0$.

Proof. All of the conclusions, except those concerning $\mathfrak{D}$, follow directly from Theorem 2.13 . When $k=0$, the trace $\mathfrak{D}^{0} \phi(M)=\phi(M)$ is well-defined, since $\varphi(R)=\varphi\left(r_{0}(M)\right)$ is well-defined. Note that since $\partial_{x} r_{0}(x)=1 /\left(4 \pi \rho_{0}(x) r_{0}^{2}(x)\right)$, we have

$$
\mathfrak{D} \phi(x)=\rho_{0}(x) \partial_{x} \phi(x)=\frac{\partial_{z} \varphi\left(r_{0}(x)\right)}{4 \pi r_{0}^{2}(x)} \Longrightarrow \mathfrak{D} \phi(M)=\frac{\partial_{z} \varphi(R)}{4 \pi R^{2}},
$$

so that $\mathfrak{D} \phi(M)$ is well-defined. In other words, the multiplication by $\rho_{0}$ in the operator $\mathfrak{D}$ removes the potential singularity in $\partial_{x} \phi$ near $x=M$. We may argue similarly, using the fact that $\partial_{z}^{k} \varphi(R)$ is well-defined for all $k \geq 0$, to deduce that $\mathfrak{D}^{k} \phi(M)$ is well-defined for all $k \geq 0$ as well.

Now, with Corollary 2.14 in hand, we are ready to present:

Proof of Theorem 2.1. Let $\lambda>0$ and $\phi(x)$ be the solution to (2-16)-(2-17) given in Corollary 2.14. Let us then define $v$ and $\sigma$ according to

$$
v=-\frac{\lambda}{4 \pi r_{0}^{2}} \phi \quad \text { and } \quad \sigma=\rho_{0}^{2} \partial_{x} \phi
$$

Using these definitions of $v$ and $\sigma$ in conjunction with the properties of $\phi$ recorded in Corollary 2.14, we easily deduce items (1)-(3).

To prove the variational characterization of item (4), we return to the variational characterization of $\lambda$ in $z=r_{0}(x)$ coordinates. According to Theorem 2.13, $\lambda>0$ satisfies

$\lambda \int_{0}^{R}\left(\delta \frac{\left|\partial_{z} \vartheta\right|^{2}}{z^{2}}+\frac{4 \varepsilon}{3 z^{2}}\left|\partial_{z} \vartheta-3 \frac{\vartheta}{z}\right|^{2}\right) d z+\int_{0}^{R}\left(\gamma P_{0} \frac{\left|\partial_{z} \vartheta\right|^{2}}{z^{2}}+4 \frac{\partial_{z} P_{0}}{z^{3}}|\vartheta|^{2}\right) d z \geq-\lambda^{2} \int_{0}^{R} \frac{\varrho_{0}}{z^{2}}|\vartheta|^{2} d z$

for every $\vartheta \in H_{2}^{1}((0, R))$. Then the variational characterization in (2-12) follows by making a change of coordinates $\theta(x)=\vartheta(z)=\vartheta\left(r_{0}(x)\right)$. Note that $\vartheta \in H_{2}^{1}((0, R))$ if and only if $\sqrt{\rho_{0}} \partial_{x} \theta \in L^{2}((0, M))$ and $\theta /\left(r_{0}^{2} \sqrt{\rho_{0}}\right) \in L^{2}((0, M))$. Also, changing coordinates in (2-26) of Lemma 2.2 shows that $\theta /\left(r_{0}^{3} \sqrt{\rho_{0}}\right) \in$ $L^{2}((0, M))$, which means that all of the integrals in (2-12) are well-defined.

We now turn to the proof of (2-13). Using the inclusion $\varphi \in H_{2}^{1}((0, R))$, the above analysis implies that $\sqrt{\rho_{0}} \partial_{x} \phi, \phi /\left(r_{0}^{3} \sqrt{\rho_{0}}\right) \in L^{2}((0, M))$. From this and (2-16), we may then deduce that

$$
\int_{0}^{M}\left(\frac{|\phi|^{2}}{r_{0}^{6} \rho_{0}}+\rho_{0}\left|\partial_{x} \phi\right|^{2}+r_{0}^{2}\left|\partial_{x}\left(\rho_{0} \partial_{x} \phi\right)\right|^{2}\right) d x<\infty .
$$

This and (2-86) then imply (2-13). 


\section{Linear estimates}

Due to the indirect way in which we constructed growing mode solutions in Section 2, it is not immediately obvious that the $\lambda>0$ of Theorem 2.1 is the largest possible growth rate. However, because of the inequality (2-12), we can show that no solution to the linearized problem (2-1)-(2-4) can grow in time at a rate faster than $e^{\lambda t}$. Hence the growing mode constructed in Theorem 2.1 actually does grow in time at the fastest possible rate. The proof of this result and its implications for solutions to the inhomogeneous linearized problem are the subject of this section.

3A. Estimates in the second-order formulation. First we will prove estimates for solutions to the following second-order problem.

$$
-\frac{\partial_{t}^{2} \phi}{16 \pi^{2} r_{0}^{4}}=\frac{\partial_{x} P_{0}}{\pi r_{0}^{3}} \phi-\partial_{x}\left[\left(\frac{4 \varepsilon}{3}+\delta\right) \rho_{0} \partial_{x} \partial_{t} \phi+\gamma P_{0} \rho_{0} \partial_{x} \phi\right] \text { for } x \in(0, M),
$$

with boundary conditions

$$
\phi(0, t)=0 \quad \text { and } \quad \frac{4 \varepsilon}{3}\left(4 \pi r_{0}^{3} \rho_{0} \partial_{x}\left(\frac{\phi}{r_{0}^{3}}\right)\right)+\delta\left(4 \pi \rho_{0} \partial_{x} \phi\right)=0 \quad \text { at } x=M,
$$

and initial conditions $\phi(x, 0)$ and $\partial_{t} \phi(x, 0)$ given. We will assume throughout that $\phi$ satisfies $\sqrt{\rho_{0}} \partial_{x} \phi \in$ $L^{2}((0, M))$ and $\phi /\left(r_{0}^{2} \sqrt{\rho_{0}}\right) \in L^{2}((0, M))$.

Solutions to this linear problem obey an energy evolution equation related to the inequality (2-12). We record this now.

Proposition 3.1. Suppose $\phi$ is a solution to (3-1)-(3-2). Then

$$
\begin{aligned}
\partial_{t} \int_{0}^{M} \frac{\left|\partial_{t} \phi\right|^{2}}{32 \pi^{2} r_{0}^{4}} d x+\int_{0}^{M}\left(\delta \rho_{0}\left|\partial_{x} \partial_{t} \phi\right|^{2}+\frac{4 \varepsilon}{3} \rho_{0}\left|r_{0}^{3} \partial_{x}\left(\frac{\partial_{t} \phi}{r_{0}^{3}}\right)\right|^{2}\right) d x & \\
& =-\partial_{t} \int_{0}^{M}\left(\frac{\gamma P_{0} \rho_{0}}{2}\left|\partial_{x} \phi\right|^{2}+\frac{\partial_{x} P_{0}}{2 \pi r_{0}^{3}}|\phi|^{2}\right) d x .
\end{aligned}
$$

Proof. Multiply (3-1) by $\partial_{t} \phi$ and integrate over $x \in(0, M)$. An integration by parts, an application of the boundary conditions (3-2), and some simple algebra yield the desired equality.

We can use this and the variational characterization of $\lambda$ given in Theorem 2.1 to deduce some estimates.

Theorem 3.2. Let $\phi$ solve (3-1)-(3-2). Then we have the following estimates:

$$
\begin{aligned}
\int_{0}^{M} \frac{|\phi(t)|^{2}}{16 \pi^{2} r_{0}^{4}} d x+\int_{0}^{t} \int_{0}^{M}\left(\delta \rho_{0}\left|\partial_{x} \phi(s)\right|^{2}+\frac{4 \varepsilon}{3} \rho_{0}\left|r_{0}^{3} \partial_{x}\left(\frac{\phi(s)}{r_{0}^{3}}\right)\right|^{2}\right) d x d s & \leq e^{2 \lambda t} \int_{0}^{M} \frac{|\phi(0)|^{2}}{16 \pi^{2} r_{0}^{4}} d x+\frac{K_{1}}{2 \lambda}\left(e^{2 \lambda t}-1\right)
\end{aligned}
$$




$$
\begin{aligned}
\frac{1}{\lambda} \int_{0}^{M} \frac{\left|\partial_{t} \phi(t)\right|^{2}}{16 \pi^{2} r_{0}^{4}} d x+\int_{0}^{M}\left(\delta \rho_{0}\left|\partial_{x} \phi(t)\right|^{2}+\frac{4 \varepsilon}{3} \rho_{0}\left|r_{0}^{3} \partial_{x}\left(\frac{\phi(t)}{r_{0}^{3}}\right)\right|^{2}\right) d x & \leq e^{2 \lambda t}\left(2 \lambda \int_{0}^{M} \frac{|\phi(0)|^{2}}{16 \pi^{2} r_{0}^{4}} d x+K_{1}\right)
\end{aligned}
$$

and

$$
\frac{1}{2} \int_{0}^{M} \gamma P_{0} \rho_{0}\left|\partial_{x} \phi(t)\right|^{2} d x \leq K_{0}+C_{0}\left[e^{2 \lambda t} \int_{0}^{M} \frac{|\phi(0)|^{2}}{16 \pi^{2} r_{0}^{4}} d x+\frac{K_{1}}{2 \lambda}\left(e^{2 \lambda t}-1\right)\right]
$$

Here

$$
\begin{aligned}
& K_{0}=\int_{0}^{M} \frac{\left|\partial_{t} \phi(0)\right|^{2}}{16 \pi^{2} r_{0}^{4}} d x+\frac{1}{2} \int_{0}^{M} \gamma P_{0} \rho_{0}\left|\partial_{x} \phi(0)\right|^{2} d x \\
& K_{1}=\frac{2 K_{0}}{\lambda}+2 \int_{0}^{M}\left(\delta \rho_{0}\left|\partial_{x} \phi(0)\right|^{2}+\frac{4 \varepsilon}{3} \rho_{0}\left|r_{0}^{3} \partial_{x}\left(\frac{\phi(0)}{r_{0}^{3}}\right)\right|^{2}\right) d x,
\end{aligned}
$$

and

$$
C_{0}=2 \sup _{x \in(0, M)} \frac{x}{r_{0}^{3}(x)}<\infty
$$

Proof. We integrate the result of Proposition 3.1 in time from 0 to $t$ to see that

$$
\begin{aligned}
\int_{0}^{M} \frac{\left|\partial_{t} \phi(t)\right|^{2}}{32 \pi^{2} r_{0}^{4}} d x+ & \int_{0}^{t} \int_{0}^{M}\left(\delta \rho_{0}\left|\partial_{x} \partial_{t} \phi(s)\right|^{2}+\frac{4 \varepsilon}{3} \rho_{0}\left|r_{0}^{3} \partial_{x}\left(\frac{\partial_{t} \phi(s)}{r_{0}^{3}}\right)\right|^{2}\right) d x d s \\
& =K_{0}+\int_{0}^{M} \frac{\partial_{x} P_{0}}{2 \pi r_{0}^{3}}|\phi(0)|^{2} d x-\int_{0}^{M}\left(\frac{\gamma P_{0} \rho_{0}}{2}\left|\partial_{x} \phi(t)\right|^{2}+\frac{\partial_{x} P_{0}}{2 \pi r_{0}^{3}}|\phi(t)|^{2}\right) d x
\end{aligned}
$$

Note that since

$$
\partial_{x} P_{0}=-\frac{x}{4 \pi r_{0}^{4}}
$$

we have

$$
\int_{0}^{M} \frac{\partial_{x} P_{0}}{2 \pi r_{0}^{3}}|\phi(0)|^{2} d x=-\int_{0}^{M} \frac{x}{8 \pi^{2} r_{0}^{7}}|\phi(0)|^{2} d x \leq 0 .
$$

The variational characterization of $\lambda$ given in (2-12) of Theorem 2.1 allows us to estimate

$$
\begin{array}{r}
-\frac{1}{2} \int_{0}^{M}\left(\frac{\gamma P_{0} \rho_{0}}{2}\left|\partial_{x} \phi(t)\right|^{2}+\frac{\partial_{x} P_{0}}{2 \pi r_{0}^{3}}|\phi(t)|^{2}\right) d x-\frac{\lambda}{2} \int_{0}^{M}\left(\delta \rho_{0}\left|\partial_{x} \phi(t)\right|^{2}+\frac{4 \varepsilon}{3} \rho_{0}\left|r_{0}^{3} \partial_{x}\left(\frac{\phi(t)}{r_{0}^{3}}\right)\right|^{2}\right) d x \\
\leq \frac{\lambda^{2}}{2} \int_{0}^{M} \frac{|\phi(t)|^{2}}{16 \pi^{2} r_{0}^{4}} d x
\end{array}
$$


We may then combine (3-10)-(3-12) to see that

$$
\begin{aligned}
& \int_{0}^{M} \frac{\left|\partial_{t} \phi(t)\right|^{2}}{32 \pi^{2} r_{0}^{4}} d x+\int_{0}^{t} \int_{0}^{M}\left(\delta \rho_{0}\left|\partial_{x} \partial_{t} \phi(s)\right|^{2}+\frac{4 \varepsilon}{3} \rho_{0}\left|r_{0}^{3} \partial_{x}\left(\frac{\partial_{t} \phi(s)}{r_{0}^{3}}\right)\right|^{2}\right) d x d s \\
& \leq K_{0}+\frac{\lambda^{2}}{2} \int_{0}^{M} \frac{|\phi(t)|^{2}}{16 \pi^{2} r_{0}^{4}} d x+\frac{\lambda}{2} \int_{0}^{M}\left(\delta \rho_{0}\left|\partial_{x} \phi(t)\right|^{2}+\frac{4 \varepsilon}{3} \rho_{0}\left|r_{0}^{3} \partial_{x}\left(\frac{\phi(t)}{r_{0}^{3}}\right)\right|^{2}\right) d x .
\end{aligned}
$$

For the sake of brevity in the rest of the proof, we now rewrite (3-13) as

$$
\frac{1}{2}\left\|\partial_{t} \phi(t)\right\|_{1}^{2}+\int_{0}^{t}\left\|\partial_{t} \phi(s)\right\|_{2}^{2} d s \leq K_{0}+\frac{\lambda^{2}}{2}\|\phi(t)\|_{1}^{2}+\frac{\lambda}{2}\|\phi(t)\|_{2}^{2}
$$

for the two norms $\|\cdot\|_{1}$ and $\|\cdot\|_{2}$ given by

$$
\begin{aligned}
\|\psi\|_{1}^{2} & :=\int_{0}^{M} \frac{|\psi|^{2}}{16 \pi^{2} r_{0}^{4}} d x \\
\|\psi\|_{2}^{2} & :=\int_{0}^{M}\left(\delta \rho_{0}\left|\partial_{x} \psi\right|^{2}+\frac{4 \varepsilon}{3} \rho_{0}\left|r_{0}^{3} \partial_{x}\left(\frac{\psi}{r_{0}^{3}}\right)\right|^{2}\right) d x .
\end{aligned}
$$

Both of these norms are clearly generated by inner products, which we will write as $\langle\cdot, \cdot\rangle_{i}$ for $i=1,2$. Integrating in time and using Cauchy's inequality, we may write the bound

$$
\begin{aligned}
\lambda\|\phi(t)\|_{2}^{2} & =\lambda\|\phi(0)\|_{2}^{2}+\lambda \int_{0}^{t} 2\left\langle\phi(s), \partial_{t} \phi(s)\right\rangle_{2} d s \\
& \leq \lambda\|\phi(0)\|_{2}^{2}+\int_{0}^{t}\left\|\partial_{t} \phi(s)\right\|_{2}^{2} d s+\lambda^{2} \int_{0}^{t}\|\phi(s)\|_{2}^{2} d s .
\end{aligned}
$$

On the other hand,

$$
\lambda \partial_{t}\|\phi(t)\|_{1}^{2}=\lambda 2\left\langle\partial_{t} \phi(t), \phi(t)\right\rangle_{1} \leq \lambda^{2}\|\phi(t)\|_{1}^{2}+\left\|\partial_{t} \phi(t)\right\|_{1}^{2}
$$

We may combine these two inequalities with (3-14) to derive the differential inequality

$$
\partial_{t}\|\phi(t)\|_{1}^{2}+\|\phi(t)\|_{2}^{2} \leq K_{1}+2 \lambda\|\phi(t)\|_{1}^{2}+2 \lambda \int_{0}^{t}\|\phi(s)\|_{2}^{2} d s
$$

for $K_{1}$ as defined in the hypotheses. An application of Gronwall's lemma then shows that

$$
\|\phi(t)\|_{1}^{2}+\int_{0}^{t}\|\phi(s)\|_{2}^{2} d s \leq e^{2 \lambda t}\|\phi(0)\|_{1}^{2}+\frac{K_{1}}{2 \lambda}\left(e^{2 \lambda t}-1\right)
$$

for all $t \geq 0$, which is the bound (3-4).

To derive the estimate (3-5), we return to (3-14) and plug in (3-17) and (3-20) to see that

$$
\frac{1}{\lambda}\left\|\partial_{t} \phi(t)\right\|_{1}^{2}+\|\phi(t)\|_{2}^{2} \leq K_{1}+\lambda\|\phi(t)\|_{1}^{2}+2 \lambda \int_{0}^{t}\|\phi(s)\|_{2}^{2} d s \leq e^{2 \lambda t}\left(2 \lambda\|\phi(0)\|_{1}^{2}+K_{1}\right) .
$$


Finally, for (3-6), we return to (3-10) and employ (3-11) to see that

$$
\frac{1}{2} \int_{0}^{M} \gamma P_{0} \rho_{0}\left|\partial_{x} \phi(t)\right|^{2} d x \leq K_{0}-\int_{0}^{M} \frac{\partial_{x} P_{0}}{2 \pi r_{0}^{3}}|\phi(t)|^{2} d x=K_{0}+\int_{0}^{M} \frac{x}{8 \pi^{2} r_{0}^{7}}|\phi(t)|^{2} d x .
$$

Since L'Hospital's theorem implies that

$$
\lim _{x \rightarrow 0} \frac{x}{r_{0}^{3}(x)}=\lim _{x \rightarrow 0} \frac{4 \pi \rho_{0}(x)}{3}=\frac{4 \pi \rho_{0}(0)}{3}<\infty,
$$

we may deduce that

$$
\sup _{x \in(0, M)} \frac{x}{r_{0}^{3}(x)}<\infty
$$

The estimate (3-6) then follows directly from (3-22), (3-24), and the estimate of $\|\phi(t)\|_{1}^{2}$ in (3-4).

3B. Estimates in the first-order formulation. Now consider $\sigma$ and $w$ to be solutions to the first-order linear system (2-5) with boundary conditions (2-6) and initial conditions $\sigma(x, 0)$ and $w(x, 0)$. A simple calculation shows that if we apply $\partial_{t}$ to the second equation in (2-5) and then eliminate $\partial_{t} \sigma$ by using the first equation in (2-5), then we arrive at the second-order formulation (3-1)-(3-2) for $\phi=w$. Then Theorem 3.2 yields various estimates for $w=\phi$. We now seek to rewrite these estimates for $w$ and to use them to derive a similar estimate for $\sigma$.

Theorem 3.3. Let $\sigma, w$ solve the linear system (2-5)-(2-6). Then

$$
\begin{array}{r}
\int_{0}^{M} \frac{|w(t)|^{2}}{r_{0}^{4}} d x+\int_{0}^{M} \gamma K \rho_{0}^{\gamma-1}\left|\frac{\sigma(t)}{\rho_{0}}\right|^{2} d x \\
\leq C e^{2 \lambda t}\left[\int_{0}^{M} \frac{|w(0)|^{2}}{r_{0}^{4}} d x+\int_{0}^{M}\left(\gamma K \rho_{0}^{\gamma-1}\left|\frac{\sigma(0)}{\rho_{0}}\right|^{2}+\gamma K \rho_{0}\left|\partial_{x} w(t)\right|^{2}+\left.\frac{4 \varepsilon}{3} \rho_{0}\left|r_{0}^{3} \partial_{x}\left(\frac{w(t)}{r_{0}^{3}}\right)\right|^{2} w(0)\right|^{2}+\frac{\left|\partial_{t} w(0)\right|^{2}}{r_{0}^{4}}\right) d x\right. \\
\left.+\int_{0}^{M}\left(\delta \rho_{0}\left|\partial_{x} w(0)\right|^{2}+\frac{4 \varepsilon}{3} \rho_{0}\left|r_{0}^{3} \partial_{x}\left(\frac{w(0)}{r_{0}^{3}}\right)\right|^{2}\right) d x\right] .
\end{array}
$$

Proof. We switch to the second-order formulation for $\phi=w$. Then the estimates (3-4)-(3-6) of Theorem 3.2 imply that

$$
\begin{aligned}
& \int_{0}^{M} \frac{|w(t)|^{2}}{r_{0}^{4}} d x+\int_{0}^{M} \gamma K \rho_{0}^{\gamma+1}\left|\partial_{x} w(t)\right|^{2} d x+16 \pi^{2} \int_{0}^{M}\left(\delta \rho_{0}\left|\partial_{x} w(t)\right|^{2}+\frac{4 \varepsilon}{3} \rho_{0}\left|r_{0}^{3} \partial_{x}\left(\frac{w(t)}{r_{0}^{3}}\right)\right|^{2}\right) d x \\
& \leq C e^{2 \lambda t}\left[\int_{0}^{M} \frac{|w(0)|^{2}}{r_{0}^{4}} d x+\right. \int_{0}^{M}\left(\gamma K \rho_{0}^{\gamma+1}\left|\partial_{x} w(0)\right|^{2}+\frac{\left|\partial_{t} w(0)\right|^{2}}{r_{0}^{4}}\right) d x \\
&\left.+\int_{0}^{M}\left(\delta \rho_{0}\left|\partial_{x} w(0)\right|^{2}+\frac{4 \varepsilon}{3} \rho_{0}\left|r_{0}^{3} \partial_{x}\left(\frac{w(0)}{r_{0}^{3}}\right)\right|^{2}\right) d x\right] .
\end{aligned}
$$

Let us call the term in the brackets on the right side of this equation $\mathscr{L}_{0}$. Since $\partial_{t} \sigma=-4 \pi \rho_{0}^{2} \partial_{x} w$, we then have that 


$$
\int_{0}^{M} \gamma K \rho_{0}^{\gamma+1}\left|\partial_{x} w(t)\right|^{2} d x=\int_{0}^{M} \frac{\gamma K \rho_{0}^{\gamma-1}}{16 \pi^{2} \rho_{0}^{2}}\left|\partial_{t} \sigma(t)\right|^{2} d x
$$

The right side of (3-27) defines the square of a norm $\|\cdot\|$ in a Hilbert space, and in this case CauchySchwarz and the chain rule imply that $\partial_{t}\|\psi(t)\| \leq\left\|\partial_{t} \psi(t)\right\|$ for a one-parameter family $\psi(t)$ in the space. Using this, we then have that

$$
\partial_{t}\left(\int_{0}^{M} \frac{\gamma K \rho_{0}^{\gamma-1}}{16 \pi^{2} \rho_{0}^{2}}|\sigma(t)|^{2} d x\right)^{1 / 2} \leq\left(\int_{0}^{M} \frac{\gamma K \rho_{0}^{\gamma-1}}{16 \pi^{2} \rho_{0}^{2}}\left|\partial_{t} \sigma(t)\right|^{2} d x\right)^{1 / 2} \leq \sqrt{C \mathscr{E}} e^{\lambda t} .
$$

Integrating this in time, we then find that

$$
\begin{aligned}
\left(\int_{0}^{M} \frac{\gamma K \rho_{0}^{\gamma-1}}{16 \pi^{2} \rho_{0}^{2}}|\sigma(t)|^{2} d x\right)^{1 / 2} & \leq\left(\int_{0}^{M} \frac{\gamma K \rho_{0}^{\gamma-1}}{16 \pi^{2} \rho_{0}^{2}}|\sigma(0)|^{2} d x\right)^{1 / 2}+\frac{\sqrt{C \mathscr{W}}}{\lambda}\left(e^{\lambda t}-1\right) \\
& \leq C e^{\lambda t} \sqrt{\int_{0}^{M} \frac{\gamma K \rho_{0}^{\gamma-1}}{16 \pi^{2} \rho_{0}^{2}}|\sigma(0)|^{2} d x+\mathscr{L}_{0} .}
\end{aligned}
$$

The estimate (3-25) then follows directly from (3-26), (3-27), and (3-29).

3C. Estimates for the inhomogeneous first-order problem. Consider the linear operators

$$
\begin{aligned}
& \mathscr{L}_{1} w=4 \pi \rho_{0}^{2} \partial_{x} w, \\
& \mathscr{L}_{2} \sigma=4 \pi r_{0}^{4} \partial_{x}\left(\gamma K \rho_{0}^{\gamma-1} \sigma\right)-4 r_{0} \partial_{x} P_{0} \int_{0}^{x} \frac{\sigma(y)}{\rho_{0}^{2}(y)} d y, \\
& \mathscr{L}_{3} w=-16 \pi^{2} r_{0}^{4} \partial_{x}\left[\left(\frac{4 \varepsilon}{3}+\delta\right) \rho_{0} \partial_{x} w\right],
\end{aligned}
$$

and the corresponding matrix of operators

$$
\mathscr{L}=\left(\begin{array}{cc}
0 & -\mathscr{L}_{1} \\
-\mathscr{L}_{2} & -\mathscr{L}_{3}
\end{array}\right)
$$

We also consider the boundary operator

$$
\mathscr{B}(w)=-\frac{4 \varepsilon}{3}\left(4 \pi r_{0}^{3} \rho_{0} \partial_{x}\left(\frac{w}{r_{0}^{3}}\right)\right)-\delta\left(4 \pi \rho_{0} \partial_{x} w\right) .
$$

Notice that the first-order equations (2-5)-(2-6) are equivalent to the equation

$$
\partial_{t}\left(\begin{array}{c}
\sigma \\
w
\end{array}\right)-\mathscr{L}\left(\begin{array}{c}
\sigma \\
w
\end{array}\right)=\left(\begin{array}{l}
0 \\
0
\end{array}\right)
$$

with homogeneous boundary conditions

$$
\frac{w}{r_{0}^{2}}(0, t)=\sigma(M, t)=0 \quad \text { and } \quad \mathscr{B}(w)=0 \quad \text { at } x=M .
$$


Let us by denote $e^{t \mathscr{L}}$ the solution operator to (3-35)-(3-36), that is,

$$
e^{t \mathscr{L}}\left(\begin{array}{c}
\sigma(0) \\
w(0)
\end{array}\right)=\left(\begin{array}{c}
\sigma(t) \\
w(t)
\end{array}\right),
$$

where $\sigma$ and $w$ solve (3-35)-(3-36) with initial data $\sigma(0)$ and $w(0)$. Note that below in (3-47) we show this operator is bounded.

Suppose now that $\sigma$ and $w$ solve the inhomogeneous problem

$$
\partial_{t}\left(\begin{array}{c}
\sigma \\
w
\end{array}\right)-\mathscr{L}\left(\begin{array}{l}
\sigma \\
w
\end{array}\right)=\left(\begin{array}{l}
N_{1} \\
N_{2}
\end{array}\right)
$$

along with the boundary conditions

$$
\frac{w}{r_{0}^{2}}(0, t)=\sigma(M, t)=0 \quad \text { and } \quad \mathscr{B}(w)=N_{\mathscr{B}} \quad \text { at } x=M .
$$

Here we assume that $N_{1}=N_{1}(x, t), N_{2}=N_{2}(x, t)$, but that $N_{\mathscr{R}}=N_{\mathscr{R}}(t)$, that is, the boundary inhomogeneity only depends on time. In order to use the linear theory we have developed, we must rewrite this as a system with homogeneous boundary conditions. To accomplish this, we will utilize the following lemma.

Lemma 3.4. Let

$$
\psi(x, t)=-\frac{N_{\mathscr{B}}(t)}{3 \delta} r_{0}^{3}(x) .
$$

Then for each $t, \psi(t)$ satisfies $\mathscr{L}_{3} \psi(t)=0$ for $x \in(0, M)$ and $\mathscr{B} \psi(t)=N_{\mathscr{B}}(t)$ at $x=M$. Also, $\mathscr{L}_{1} \psi(t)=-N_{\mathscr{B}}(t) \rho_{0}(x) / \delta$.

Proof. The results follow from simple computations.

With this $\psi$ in hand, we can reformulate (3-38)-(3-39) so that the resulting problem has homogeneous boundary conditions. Let $w=\psi+\bar{w}$. Then Lemma 3.4 implies that

$$
\partial_{t}\left(\begin{array}{c}
\sigma \\
\bar{w}
\end{array}\right)-\mathscr{L}\left(\begin{array}{c}
\sigma \\
\bar{w}
\end{array}\right)=\left(\begin{array}{l}
N_{1} \\
N_{2}
\end{array}\right)+\left(\begin{array}{c}
-\mathscr{L}_{1} \psi \\
-\partial_{t} \psi
\end{array}\right)=\left(\begin{array}{c}
N_{1}+\frac{N_{\mathscr{B}} \rho_{0}}{\delta} \\
N_{2}+\frac{\partial_{t} N_{\overparen{B}} r_{0}^{3}}{3 \delta}
\end{array}\right),
$$

along with the boundary conditions

$$
\frac{\bar{w}}{r_{0}^{2}}(0, t)=\sigma(M, t)=0 \quad \text { and } \quad \mathscr{B}(\bar{w})=0 \quad \text { at } x=M .
$$

Employing the variation of parameters, we can then solve (3-41)-(3-42) via

$$
\left(\begin{array}{c}
\sigma(t) \\
\bar{w}(t)
\end{array}\right)=e^{t \mathscr{L}}\left(\begin{array}{c}
\sigma(0) \\
\bar{w}(0)
\end{array}\right)+\int_{0}^{t} e^{(t-s) \mathscr{L}}\left(\begin{array}{c}
N_{1}(s) \\
N_{2}(s)
\end{array}\right) d s+\frac{1}{\delta} \int_{0}^{t} e^{(t-s) \mathscr{L}}\left(\begin{array}{c}
N_{\mathscr{B}}(s) \rho_{0} \\
\frac{1}{3} \partial_{t} N_{\mathscr{B}}(s) r_{0}^{3}
\end{array}\right) d s .
$$


We can then go back to $w=\psi+\bar{w}$ :

$$
\begin{aligned}
\left(\begin{array}{l}
\sigma(t) \\
w(t)
\end{array}\right)=e^{t \mathscr{L}}\left(\begin{array}{l}
\sigma(0) \\
\bar{w}(0)
\end{array}\right)-\frac{1}{\delta}\left(\begin{array}{c}
0 \\
\frac{1}{3} N_{\mathscr{B}}(t) r_{0}^{3}
\end{array}\right) & \quad+\int_{0}^{t} e^{(t-s) \mathscr{L}}\left(\begin{array}{l}
N_{1}(s) \\
N_{2}(s)
\end{array}\right) d s+\frac{1}{\delta} \int_{0}^{t} e^{(t-s) \mathscr{L}}\left(\begin{array}{c}
N_{\mathscr{B}_{B}}(s) \rho_{0} \\
\frac{1}{3} \partial_{t} N_{\mathscr{B}}(s) r_{0}^{3}
\end{array}\right) d s .
\end{aligned}
$$

Now let us define a norm for the pair $\sigma, w$ given by

$$
\begin{aligned}
\left\|\left(\begin{array}{c}
\sigma \\
w
\end{array}\right)\right\|_{0}^{2}:=\frac{1}{2} \int_{0}^{M} \gamma K \rho_{0}^{\gamma-1}\left|\frac{\sigma}{\rho_{0}}\right|^{2} d x+ & \frac{1}{2} \int_{0}^{M} \frac{|w|^{2}}{r_{0}^{4}} d x \\
& +\frac{1}{2} \int_{0}^{M} 16 \pi^{2}\left(\delta \rho_{0}\left|\partial_{x} w\right|^{2}+\frac{4 \varepsilon}{3} \rho_{0}\left|r_{0}^{3} \partial_{x}\left(\frac{w}{r_{0}^{3}}\right)\right|^{2}\right) d x .
\end{aligned}
$$

We also define

$$
\mathfrak{E}(\sigma, w):=\left\|\left(\begin{array}{c}
\sigma \\
w
\end{array}\right)\right\|_{0}^{2}+\frac{1}{2} \int_{0}^{M} \gamma K \rho_{0}^{\gamma-1}\left|\frac{\partial_{t} \sigma}{\rho_{0}}\right|^{2} d x+\frac{1}{2} \int_{0}^{M} \frac{\left|\partial_{t} w\right|^{2}}{r_{0}^{4}} d x
$$

We can then recast the result of Theorem 3.3 as

$$
\left\|e^{t \mathscr{L}}\left(\begin{array}{c}
\sigma(0) \\
w(0)
\end{array}\right)\right\|_{0}^{2} \leq C e^{2 \lambda t} \mathfrak{E}(\sigma(0), w(0)) .
$$

Using these quantities and estimate (3-47), we can record estimates for solutions to (3-38)-(3-39).

Theorem 3.5. Suppose that $\sigma$ and $w$ solve the inhomogeneous linear problem (3-38)-(3-39). Let $\psi$ be given by Lemma 3.4 and $\bar{w}=w-\psi$. Let $\|\cdot\|_{0}$ and $\mathfrak{E}(\cdot, \cdot)$ be given by (3-45) and (3-46), respectively. Then

$$
\begin{aligned}
\left\|\left(\begin{array}{c}
\sigma(t) \\
w(t)
\end{array}\right)-e^{t \mathscr{L}}\left(\begin{array}{c}
\sigma(0) \\
\bar{w}(0)
\end{array}\right)\right\|_{0} \leq & \int_{0}^{t} C e^{\lambda(t-s)} \sqrt{\mathfrak{E}\left(N_{1}(s), N_{2}(s)\right)} d s \\
& +\frac{C}{\delta}\left|N_{\mathscr{B}}(t)\right|+\frac{C}{\delta} \int_{0}^{t} e^{\lambda(t-s)}\left(\left|N_{\mathscr{B}}(s)\right|+\left|\partial_{t} N_{\mathscr{B}}(s)\right|+\left|\partial_{t}^{2} N_{\mathscr{B}}(s)\right|\right) d s .
\end{aligned}
$$

Proof. From the above analysis, we know that $\sigma$ and $w$ are given by (3-44), where $e^{t \mathscr{L}}$ is the homogeneous solution operator given by (3-37). Hence (3-47) implies that

$$
\begin{aligned}
\left\|\left(\begin{array}{c}
\sigma(t) \\
w(t)
\end{array}\right)-e^{t \mathscr{L}}\left(\begin{array}{c}
\sigma(0) \\
\bar{w}(0)
\end{array}\right)\right\|_{0} & \leq \int_{0}^{t} C e^{\lambda(t-s)} \sqrt{\mathfrak{E}\left(N_{1}(s), N_{2}(s)\right)} d s \\
+ & \frac{1}{\delta}\left\|\left(\begin{array}{c}
0 \\
\frac{1}{3} N_{\mathscr{B}}(t) r_{0}^{3}
\end{array}\right)\right\|_{0}+\frac{1}{\delta} \int_{0}^{t} C e^{\lambda(t-s)} \sqrt{\mathfrak{E}\left(N_{\mathscr{B}}(s) \rho_{0}, \frac{1}{3} \partial_{t} N_{\mathscr{B}}(s) r_{0}^{3}\right)} d s .
\end{aligned}
$$

Then, since $N_{\mathscr{B}}(t)$ is only a function of time, not of $x$, we can easily estimate

$$
\left\|\left(\begin{array}{c}
0 \\
\frac{1}{3} N_{\mathscr{B}}(t) r_{0}^{3}
\end{array}\right)\right\|_{0} \leq C\left|N_{\mathscr{B}}(t)\right|
$$


and

$$
\sqrt{\mathfrak{E}\left(N_{\mathscr{B}}(s) \rho_{0}, \frac{1}{3} \partial_{t} N_{\mathscr{B}}(s) r_{0}^{3}\right)} \leq C\left(\left|N_{\mathscr{B}}(s)\right|+\left|\partial_{t} N_{\mathscr{B}}(s)\right|+\left|\partial_{t}^{2} N_{\mathscr{B}}(s)\right|\right),
$$

where $C>0$ in (3-50)-(3-51) is a constant depending on various (finite) integrals of $\rho_{0}$ and $r_{0}$. The estimate (3-48) then follows by combining (3-49)-(3-51).

\section{Nonlinear energy estimates}

4A. Definitions. We are interested in small perturbations $\sigma, v$ around the stationary solution $\rho=\rho_{0}$, $r=r_{0}$, and $v=0$. In particular, we assume that

$$
\frac{9}{10} \rho_{0} \leq \rho_{0}+\sigma \leq \frac{11}{10} \rho_{0}
$$

This assumption will be justified later when we close the nonlinear energy estimates. For such small solutions, the Navier-Stokes-Poisson system (1-17) and (1-18) can be written as follows:

$$
\begin{aligned}
\partial_{t} \sigma+4 \pi \rho^{2} \partial_{x}\left(r^{2} v\right) & =0 \\
\partial_{t} v+4 \pi r^{2} \partial_{x} P-4 \pi r_{0}^{2} \partial_{x} P_{0}+\frac{x}{r^{2}}-\frac{x}{r_{0}^{2}} & =16 \pi^{2} r^{2} \partial_{x}\left(\left(\frac{4 \varepsilon}{3}+\delta\right) \rho \partial_{x}\left(r^{2} v\right)\right) .
\end{aligned}
$$

The dynamics of $r$ are determined by

$$
r(x, t)=\left(\frac{3}{4 \pi} \int_{0}^{x} \frac{d y}{\rho_{0}(y)+\sigma(y, t)}\right)^{1 / 3} \quad \text { and } \quad \partial_{t} r(x, t)=v(x, t) .
$$

It turns out that it is convenient to analyze $\frac{\sigma}{\rho_{0}}$ rather than $\sigma$ itself, so we rewrite the continuity equation as

$$
\frac{\rho_{0}}{\rho} \partial_{t}\left(\frac{\sigma}{\rho_{0}}\right)+4 \pi \rho \partial_{x}\left(r^{2} v\right)=0
$$

We will also rewrite the momentum equation. To do so, we first note that

$$
4 \pi r^{2} \partial_{x} P-4 \pi r_{0}^{2} \partial_{x} P_{0}+\frac{x}{r^{2}}-\frac{x}{r_{0}^{2}}=4 \pi r^{2} \partial_{x}\left(P-P_{0}\right)+x\left(\frac{1}{r^{2}}-\frac{r^{2}}{r_{0}^{4}}\right),
$$

and then note that for small perturbations satisfying (4-1), $P-P_{0}=K\left(\rho^{\gamma}-\rho_{0}^{\gamma}\right)$ can be written as

$$
P-P_{0}=\rho_{0}^{\gamma}\left\{K \gamma \frac{\sigma}{\rho_{0}}+a_{*}\left(\frac{\sigma}{\rho_{0}}\right)^{2}\right\},
$$

where $a_{*}$ is the smooth bounded remainder from the Taylor's theorem. We then rewrite the momentum equation as

$$
\partial_{t} v+4 \pi r^{2} \partial_{x}\left\{K \gamma \rho_{0}^{\gamma} \frac{\sigma}{\rho_{0}}+a_{*} \rho_{0}^{\gamma}\left(\frac{\sigma}{\rho_{0}}\right)^{2}\right\}+x\left(\frac{1}{r^{2}}-\frac{r^{2}}{r_{0}^{4}}\right)=\mathscr{V},
$$


where

$$
\mathscr{V}:=16 \pi^{2} r^{2} \partial_{x}\left(\left(\frac{4 \varepsilon}{3}+\delta\right) \rho \partial_{x}\left(r^{2} v\right)\right) .
$$

We give an equivalent expression for $\mathscr{V}$ so that it appreciates the boundary condition (1-20) in energy estimates:

$$
\mathscr{V}=16 \pi^{2} r^{2} \partial_{x} \mathscr{W}+\frac{4 \varepsilon}{3} 12 \pi r^{2} \partial_{x}\left(\frac{v}{r}\right),
$$

where

$$
\mathscr{W}=\delta \rho \partial_{x}\left(r^{2} v\right)+\frac{4 \varepsilon}{3} \rho r^{3} \partial_{x}\left(\frac{v}{r}\right)
$$

satisfies $\mathscr{W}(M)=0$ because of the boundary condition (1-20). We use $v$ to denote the minimal viscosity coefficient:

$$
v:=\min \left\{\delta, \frac{4 \varepsilon}{3}\right\} .
$$

We now define instant energy functionals for $\sigma$ and $v$. In what follows, all of the integrals are understood to be over the interval $[0, M]$.

$$
\begin{aligned}
\mathscr{E}^{0}:= & \frac{1}{2} \int|v|^{2} d x+\frac{1}{2} \int \frac{K \gamma \rho_{0}^{\gamma-1}}{\left(1+\frac{\sigma}{\rho_{0}}\right)^{2}}\left|\frac{\sigma}{\rho_{0}}\right|^{2} d x+\frac{1}{2} \int v\left|1-\frac{r_{0}}{r}\right|^{2} d x \\
= & : \mathscr{E}^{0, v}+\mathscr{E}^{0, \sigma}+\mathscr{E}^{0} 0, r \\
\mathscr{E}^{1}:= & \frac{1}{2}\left[\delta \int 16 \pi^{2} \rho\left|\partial_{x}\left(r^{2} v\right)\right|^{2} d x+\frac{4 \varepsilon}{3} \int 16 \pi^{2} \rho r^{6}\left|\partial_{x}\left(\frac{v}{r}\right)\right|^{2} d x\right], \\
& +\frac{1}{2} \int\left(\delta+\frac{4 \varepsilon}{3}\right) 16 \pi^{2} r^{4} \frac{1}{1+\frac{\sigma}{\rho_{0}}}\left|\partial_{x}\left(\frac{\sigma}{\rho_{0}}\right)\right|^{2} d x \\
= & : \mathscr{E}^{1, v}+\mathscr{E}^{1, \sigma}, \\
\mathscr{E}^{2}:= & \frac{1}{2} \int\left|\partial_{t} v\right|^{2} d x+\frac{1}{2} \int \frac{K \gamma \rho_{0}^{\gamma-1}}{\left(1+\frac{\sigma}{\rho_{0}}\right)^{2}}\left|\partial_{t}\left(\frac{\sigma}{\rho_{0}}\right)\right|^{2} d x \\
= & : \mathscr{E}^{2, v}+\mathscr{E}^{2, \sigma}, \\
\mathscr{E}^{3}:= & \frac{1}{2}\left[\delta \int 16 \pi^{2} \rho\left|\partial_{x}\left(r^{2} \partial_{t} v\right)\right|^{2} d x+\frac{4 \varepsilon}{3} \int 16 \pi^{2} \rho r^{6}\left|\partial_{x}\left(\frac{\partial_{t} v}{r}\right)\right|^{2} d x\right], \\
\mathscr{E}^{4}:= & \frac{1}{2} \int\left(\delta+\frac{4 \varepsilon}{3}\right) 4 \pi \rho_{0}\left|\partial_{x}\left(r^{4} \partial_{x}\left(\frac{\sigma}{\rho_{0}}\right)\right)\right|^{2} d x .
\end{aligned}
$$

The corresponding dissipations are given by 


$$
\begin{aligned}
& \mathscr{D}^{0}:=\delta \int 16 \pi^{2} \rho\left|\partial_{x}\left(r^{2} v\right)\right|^{2} d x+\frac{4 \varepsilon}{3} \int 16 \pi^{2} \rho r^{6}\left|\partial_{x}\left(\frac{v}{r}\right)\right|^{2} d x, \\
& \mathscr{D}^{1}:=\int\left|\partial_{t} v\right|^{2} d x+\int 16 \pi^{2} K \gamma r^{4} \rho_{0}^{\gamma}\left|\partial_{x}\left(\frac{\sigma}{\rho_{0}}\right)\right|^{2} d x:=\mathscr{D}^{1, v}+\mathscr{D}^{1, \sigma}, \\
& \mathscr{D}^{2}:=\delta \int 16 \pi^{2} \rho\left|\partial_{x}\left(r^{2} \partial_{t} v\right)\right|^{2} d x+\frac{4 \varepsilon}{3} \int 16 \pi^{2} \rho r^{6}\left|\partial_{x}\left(\frac{\partial_{t} v}{r}\right)\right|^{2} d x, \\
& \mathscr{D}^{3}:=\int\left|\partial_{t}^{2} v\right|^{2} d x, \\
& \mathscr{D}^{4}:=\int 4 \pi K \gamma r^{2} \rho \rho_{0}^{\gamma}\left|\partial_{x}\left(r^{4} \partial_{x}\left(\frac{\sigma}{\rho_{0}}\right)\right)\right|^{2} d x .
\end{aligned}
$$

We note that $\mathscr{C}^{0}$ in (4-11) corresponds to the physical energy given in (1-31) and $\mathscr{D}^{0}$ is the corresponding dissipation. $\mathscr{E}^{1}$ is the energy for the first spatial derivatives of $v$ and $\sigma$ and its structure comes from the viscosity term (for instance, see (4-8)), and $\mathscr{D}^{1}$ is the corresponding dissipation. $\mathscr{E}^{2}$ and $\mathscr{E}^{3}$ are the temporally higher-order energies of $\mathscr{E}^{0}$ and $\mathscr{E}^{1} \cdot \mathscr{E}^{4}$ is the energy for the second derivative of $\sigma$ and its form is closely related to the structure of the Navier-Stokes-Poisson system (4-2), which can be seen in (4-79).

In addition, we introduce various bootstrapped and auxiliary energies and dissipations (denoted by subscripts $b$ and $a$, respectively) that can be controlled with the above instant energies and dissipations:

$$
\begin{aligned}
\mathscr{E}_{b}^{0, r} & :=\int \frac{v}{\rho}\left|1-\frac{r_{0}}{r}\right|^{2} d x, \\
\mathscr{E}_{b}^{1, \sigma} & :=\frac{1}{2} \int\left(\delta+\frac{4 \varepsilon}{3}\right) 16 \pi^{2} \frac{r^{2}}{\rho}\left|\partial_{x}\left(\frac{\sigma}{\rho_{0}}\right)\right|^{2} d x, \\
\mathscr{D}_{b}^{1, \sigma} & :=\int 16 \pi^{2} K \gamma r^{2} \rho_{0}^{\gamma-1}\left|\partial_{x}\left(\frac{\sigma}{\rho_{0}}\right)\right|^{2} d x .
\end{aligned}
$$

We note that these bootstrapped energies and dissipations have similar structure to the ones without subscript $b$, but have the stronger weights $1 / \rho$ because $\rho$ vanishes at $x=M$. The control of them will allow us to have the estimates with improved weights, and it will also be helpful to obtain the higher-order estimates. The following auxiliary energies are motivated by the structure of the higher-order derivatives of the equations in (4-2); for instance, see (4-45), (4-75) and (4-79).

$$
\begin{aligned}
\mathscr{E}_{a}^{3, \sigma} & :=\int\left(\delta+\frac{4 \varepsilon}{3}\right)^{2} 16 \pi^{2} \frac{r^{2}}{\rho}\left|\partial_{x} \partial_{t}\left(\frac{\sigma}{\rho_{0}}\right)\right|^{2} d x, \\
\mathscr{E}_{a_{1}}^{3, v} & :=\int \frac{r^{2}}{\rho}\left|\partial_{x}\left(\rho \partial_{x}\left(r^{2} v\right)\right)\right|^{2} d x, \\
\mathscr{E}_{a_{2}}^{3, v} & :=\int \rho r^{6}\left|\partial_{x}\left(\rho r^{3} \partial_{x}\left(\frac{v}{r}\right)\right)\right|^{2} d x,
\end{aligned}
$$




$$
\begin{aligned}
& \mathscr{E}_{a_{1}}^{4}:=\int\left(\delta+\frac{4 \varepsilon}{3}\right) 4 \pi \rho_{0}\left|\partial_{x}\left(r^{4} \partial_{t} \partial_{x}\left(\frac{\sigma}{\rho_{0}}\right)\right)\right|^{2} d x, \\
& \mathscr{E}_{a_{2}}^{4}:=\int 16 \pi^{2} \rho_{0}\left|\partial_{x}\left(r^{4} \partial_{x}\left(\rho \partial_{x}\left(r^{2} v\right)\right)\right)\right|^{2} d x .
\end{aligned}
$$

Finally, we introduce some bootstrap energies that depend on a parameter $\beta \in \mathbb{R}$ :

$$
\mathscr{E}_{\beta}^{0, \sigma}:=\int \frac{\rho_{0}^{\beta+1}}{\rho}\left|\frac{\sigma}{\rho_{0}}\right|^{2} d x, \quad \mathscr{E}_{\beta}^{2, \sigma}:=\int \frac{\rho_{0}^{\beta+1}}{\rho}\left|\partial_{t}\left(\frac{\sigma}{\rho_{0}}\right)\right|^{2} d x .
$$

For the proof of our instability in Section 5, we will need to invoke higher-order energy functionals and dissipations, which are the higher-order generalizations of the above energies and dissipations. For $i=2$ and 3 , let

$$
\begin{aligned}
\mathscr{E}^{1+2 i} & :=\frac{1}{2} \int\left|\partial_{t}^{i} v\right|^{2} d x+\frac{1}{2} \int \frac{K \gamma \rho_{0}^{\gamma-1}}{\left(1+\frac{\sigma}{\rho_{0}}\right)^{2}}\left|\partial_{t}^{i}\left(\frac{\sigma}{\rho_{0}}\right)\right|^{2} d x, \\
\mathscr{D}^{1+2 i} & :=\delta \int 16 \pi^{2} \rho\left|\partial_{x}\left(r^{2} \partial_{t}^{i} v\right)\right|^{2} d x+\frac{4 \varepsilon}{3} \int 16 \pi^{2} \rho r^{6}\left|\partial_{x}\left(\frac{\partial_{t}^{i} v}{r}\right)\right|^{2} d x, \\
\mathscr{E}^{2+2 i} & :=\frac{1}{2}\left[\delta \int 16 \pi^{2} \rho\left|\partial_{x}\left(r^{2} \partial_{t}^{i} v\right)\right|^{2} d x+\frac{4 \varepsilon}{3} \int 16 \pi^{2} \rho r^{6}\left|\partial_{x}\left(\frac{\partial_{t}^{i} v}{r}\right)\right|^{2} d x\right], \\
\mathscr{D}^{2+2 i} & :=\int\left|\partial_{t}^{i+1} v\right|^{2} d x .
\end{aligned}
$$

Next we define bootstrapped energies and auxiliary energies for $i=2$ and 3 :

$$
\begin{aligned}
\mathscr{E}_{-1}^{1+2 i, \sigma} & :=\int \frac{1}{\rho_{0}}\left|\partial_{t}^{i}\left(\frac{\sigma}{\rho_{0}}\right)\right|^{2} d x, \quad \mathscr{E}_{a}^{1+2 i, v}:=\int r^{4}\left|\partial_{x}\left(\rho \partial_{x}\left(\partial_{t}^{i-1}\left[r^{2} v\right]\right)\right)\right|^{2} d x, \\
\mathscr{E}_{a}^{2+2 i, \sigma} & :=\int\left(\delta+\frac{4 \varepsilon}{3}\right)^{2} 16 \pi^{2} r^{2}\left|\partial_{x} \partial_{t}^{i}\left(\frac{\sigma}{\rho_{0}}\right)\right|^{2} d x .
\end{aligned}
$$

We then define the total energy by

$$
\begin{aligned}
\mathscr{E}:=\sum_{i=0}^{8} \mathscr{E}^{i}+\mathscr{E}_{b}^{0, r}+\mathscr{E}_{b}^{1, \sigma}+\mathscr{E}_{-1}^{0, \sigma} & +\mathscr{E}_{-1}^{2, \sigma}+\mathscr{E}_{-1}^{5, \sigma}+\mathscr{E}_{-1}^{7, \sigma} \\
& +\mathscr{E}_{a}^{3, \sigma}+\mathscr{E}_{a_{1}}^{3, v}+\mathscr{E}_{a_{2}}^{3, v}+\mathscr{E}_{a_{1}}^{4}+\mathscr{E}_{a_{2}}^{4}+\mathscr{E}_{a}^{5, \sigma}+\mathscr{E}_{a}^{6, \sigma}+\mathscr{E}_{a}^{7, \sigma}+\mathscr{E}_{a}^{8, \sigma} .
\end{aligned}
$$

The introduction of the above notation for the energies and dissipations is lengthy, but at each level they capture the complex structure of the Navier-Stokes-Poisson system with degeneracy of $\rho$ at $x=M$ and $r$ at $x=0$, and lead to successful energy estimates. We have separated the energies from one another because the estimate of each energy term in $\mathscr{E}$ will be derived by a different strategy and method. 
Throughout the rest of the section, we assume that

$$
\begin{aligned}
\left\|\frac{\sigma}{\rho_{0}}\right\|_{L^{\infty}}+\left\|\partial_{t}\left(\frac{\sigma}{\rho_{0}}\right)\right\|_{L^{\infty}}+\left\|\partial_{t}^{2}\left(\frac{\sigma}{\rho_{0}}\right)\right\|_{L^{\infty}}+\left\|\partial_{t}^{3}\left(\frac{\sigma}{\rho_{0}}\right)\right\|_{L^{\infty}}+\left\|1-\frac{r_{0}}{r}\right\|_{L^{\infty}} \\
+\left\|\rho r^{3} \partial_{x}\left(\frac{v}{r}\right)\right\|_{L^{\infty}}+\left\|\rho r^{3} \partial_{x}\left(\frac{\sigma}{\rho_{0}}\right)\right\|_{L^{\infty}}+\left\|\frac{v}{r}\right\|_{L^{\infty}}+\left\|\frac{\partial_{t} v}{r}\right\|_{L^{\infty}}+\left\|\frac{\partial_{t}^{2} v}{r}\right\|_{L^{\infty}} \leq \theta_{1}
\end{aligned}
$$

for sufficiently small constant $\theta_{1}$, where the norm $\|\cdot\|_{L^{\infty}}$ is over the spatial region $[0, M]$. The validity of this assumption within the total energy $\mathscr{E}$ will be justified in Lemma 4.9.

Since $r$ is determined through an integral of $\sigma$ as in (4-3), for small perturbations satisfying (4-1) we may use Taylor's theorem to write $r_{0} / r$ as

$$
\frac{r_{0}}{r}=1+\frac{1}{4 \pi r_{0}^{3}} \int_{0}^{x} \frac{\sigma}{\rho_{0}^{2}} d y+\frac{c_{1}}{r_{0}^{3}} \int_{0}^{x} \frac{1}{\rho_{*}}\left(\frac{\sigma}{\rho_{0}}\right)^{2} d y+\frac{c_{2}}{r_{0}^{6}}\left(\int_{0}^{x} \frac{\sigma}{\rho_{0}^{2}} d y\right)^{2},
$$

where $\rho_{*} / \rho_{0} \sim 1$ is a bounded smooth function of $\sigma / \rho_{0}$. Hence the $1-r_{0} / r$ estimate (up to a constant) in (4-20) can actually be guaranteed by the smallness of the other terms in (4-20).

The relation (4-21) will be useful in various places. We now record a couple other useful identities.

Dynamics of $r_{0} / r$. From (4-3), we have

$$
\begin{aligned}
& \partial_{t}\left(\frac{r_{0}}{r}\right)=-\frac{r_{0} v}{r^{2}}=-\left(\frac{v}{r}\right)\left(\frac{r_{0}}{r}\right), \\
& \partial_{x}\left(\frac{r_{0}}{r}\right)=\frac{1}{4 \pi \rho_{0} r_{0}^{2} r}-\frac{r_{0}}{4 \pi \rho r^{4}}=\frac{1-\left(\frac{r_{0}}{r}\right)^{3}+\frac{\sigma}{\rho_{0}}}{4 \pi \rho r_{0}^{2} r} .
\end{aligned}
$$

Some useful inequalities and identities. For any $v$ (not just solutions),

$$
\begin{aligned}
& \frac{v}{r}=\frac{4 \pi}{3}\left\{\rho \partial_{x}\left(r^{2} v\right)-\rho r^{3} \partial_{x}\left(\frac{v}{r}\right)\right\} \Rightarrow \frac{v^{2}}{\rho r^{2}} \leq \frac{32 \pi^{2}}{9}\left\{\rho\left|\partial_{x}\left(r^{2} v\right)\right|^{2}+\rho r^{6}\left|\partial_{x}\left(\frac{v}{r}\right)\right|^{2}\right\} \\
& \rho \partial_{x}\left(r v^{2}\right)=\rho \partial_{x}\left[\left(r^{2} v\right)^{2} \cdot \frac{1}{r^{3}}\right]=2 \frac{v}{r} \rho \partial_{x}\left(r^{2} v\right)-\frac{3}{4 \pi} \frac{v^{2}}{r^{2}}=\frac{v}{r}\left\{\rho \partial_{x}\left(r^{2} v\right)+\rho r^{3} \partial_{x}\left(\frac{v}{r}\right)\right\}
\end{aligned}
$$

4B. Estimates. Throughout the rest of the section, we use $C$ to denote a generic constant that may differ from line to line, and $\eta$ to denote a sufficiently small fixed constant which will be determined later. The constants $C$ are allowed to depend on $\eta$, which presents no trouble in our ultimate analysis since first we will fix an $\eta$, which then fixes the constants. 
In the following series of lemmas, we provide the energy inequalities for $\mathscr{E}$. We present them in the order that we use for the bootstrap argument in Section 5A. Here is the flowchart for the estimates:

$$
\begin{aligned}
\mathscr{E}^{0} & \rightarrow \mathscr{E}_{\beta}^{0, \sigma} \rightarrow \mathscr{E}^{1} \rightarrow \mathscr{E}^{2} \rightarrow \mathscr{E}_{\beta}^{2, \sigma} \rightarrow \mathscr{E}^{3} \\
& \rightarrow \mathscr{E}_{b}^{0, r} \rightarrow \mathscr{E}_{b}^{1, \sigma} \rightarrow \mathscr{E}_{a}^{3, \sigma} \rightarrow \mathscr{E}_{a_{1}}^{3, v} \rightarrow \mathscr{E}_{a_{2}}^{3, v} \\
& \rightarrow \mathscr{E}^{4} \rightarrow \mathscr{E}_{a_{1}}^{4} \rightarrow \mathscr{E}_{a_{2}}^{4} \rightarrow \mathscr{E}^{5} \rightarrow \mathscr{E}_{a}^{5} \rightarrow \mathscr{E}_{-1}^{5, \sigma} \\
& \rightarrow \mathscr{E}^{6} \rightarrow \mathscr{E}_{a}^{6, \sigma} \rightarrow \mathscr{E}^{7} \rightarrow \mathscr{E}_{a}^{7} \rightarrow \mathscr{E}_{-1}^{7, \sigma} \rightarrow \mathscr{E}^{8} \rightarrow \mathscr{E}_{a}^{8, \sigma} .
\end{aligned}
$$

We start with $\mathscr{E}^{0}$ and $\mathscr{D}^{0}$.

\section{Lemma 4.1.}

$$
\frac{d}{d t} \mathscr{E}^{0}+\mathscr{D}^{0} \leq C\left(1+\theta_{1}\right)^{\mathscr{E}} \mathscr{E}^{0}+\frac{1}{2} \mathscr{D}^{0} .
$$

Proof. Multiply (4-7) by $v$ and integrate to get

$$
\frac{1}{2} \frac{d}{d t} \int|v|^{2} d x-\underbrace{-\int 4 \pi \partial_{x}\left(r^{2} v\right)\left\{K \gamma \rho_{0}^{\gamma} \frac{\sigma}{\rho_{0}}+a_{*} \rho_{0}^{\gamma}\left(\frac{\sigma}{\rho_{0}}\right)^{2}\right\} d x}+\underbrace{\int v \frac{x\left(r_{0}^{4}-r^{4}\right)}{r^{2} r_{0}^{4}} d x}=\underbrace{\int v \mathscr{V} d x} .
$$

For (i), we use (4-4) to see that

$$
\begin{aligned}
\text { (i) } & =\int \frac{\rho_{0}}{\rho^{2}} \partial_{t}\left(\frac{\sigma}{\rho_{0}}\right)\left\{K \gamma \rho_{0}^{\gamma} \frac{\sigma}{\rho_{0}}+a_{*} \rho_{0}^{\gamma}\left(\frac{\sigma}{\rho_{0}}\right)^{2}\right\} d x \\
& =\frac{1}{2} \frac{d}{d t} \int \frac{K \gamma \rho_{0}^{\gamma-1}}{\left(1+\frac{\sigma}{\rho_{0}}\right)^{2}}\left|\frac{\sigma}{\rho_{0}}\right|^{2} d x+\int \frac{\left(K \gamma+\left(1+\frac{\sigma}{\rho_{0}}\right) a_{*}\right) \rho_{0}^{\gamma-1}}{\left(1+\frac{\sigma}{\rho_{0}}\right)^{3}}\left|\frac{\sigma}{\rho_{0}}\right|^{2} \partial_{t}\left(\frac{\sigma}{\rho_{0}}\right) d x .
\end{aligned}
$$

However,

$$
\left.\left|\int \frac{\left(K \gamma+\left(1+\frac{\sigma}{\rho_{0}}\right) a_{*}\right) \rho_{0}^{\gamma-1}}{\left(1+\frac{\sigma}{\rho_{0}}\right)^{3}}\right| \frac{\sigma}{\rho_{0}}\right|^{2} \partial_{t}\left(\frac{\sigma}{\rho_{0}}\right) d x \mid \leq C\left(1+\theta_{1}\right)^{\mathscr{E} 0}
$$

For (ii), the Cauchy-Schwarz inequality yields

$$
|(\mathrm{ii})| \leq v \int \frac{|v|^{2}}{\rho r^{2}} d x+\frac{1}{v} \int \rho\left|\frac{x}{r_{0}^{4}}\left(r^{2}+r_{0}^{2}\right)\left(r+r_{0}\right)\right|^{2}\left|1-\frac{r_{0}}{r}\right|^{2} d x \leq \frac{2}{9} \mathscr{D}^{0}+C_{\mathscr{C}}^{\operatorname{co}} 0, r,
$$

where we have used (4-23) at the second inequality. From (4-8) and the boundary condition $\mathcal{W}(M, t)=0$, we get

$$
\text { (iii) }=-\delta \int 16 \pi^{2} \rho\left|\partial_{x}\left(r^{2} v\right)\right|^{2} d x-\frac{4 \varepsilon}{3} \int 16 \pi^{2} \rho r^{6}\left|\partial_{x}\left(\frac{v}{r}\right)\right|^{2} d x=-\mathscr{D}^{0}
$$


Next, from (4-22),

$$
\begin{aligned}
\frac{v}{2} \frac{d}{d t} \int\left|1-\frac{r_{0}}{r}\right|^{2} d x & =-v \int \frac{v}{r} \frac{r_{0}}{r}\left(1-\frac{r_{0}}{r}\right) d x \\
& \leq v \int \frac{|v|^{2}}{\rho r^{2}} d x+v \int \rho\left|\frac{r_{0}}{r}\right|^{2}\left|1-\frac{r_{0}}{r}\right|^{2} d x \leq \frac{2}{9} \mathscr{D}^{0}+C_{\mathscr{E}}^{\mathscr{C}^{0}, r} .
\end{aligned}
$$

The desired estimate then follows by combining these estimates.

With Lemma 4.1, we can bootstrap to control $\frac{\sigma}{\rho_{0}}$ with an improved weight. Multiply (4-4) by $\rho_{0}^{\beta} \frac{\sigma}{\rho_{0}}$ and integrate to get

$$
\int \frac{\rho_{0}^{\beta+1}}{\rho} \frac{\sigma}{\rho_{0}} \partial_{t}\left(\frac{\sigma}{\rho_{0}}\right) d x=-\int \rho^{1 / 2} \rho_{0}^{\beta} \frac{\sigma}{\rho_{0}} \cdot 4 \pi \rho^{1 / 2} \partial_{x}\left(r^{2} v\right) d x
$$

Thus

$\frac{1}{2} \frac{d}{d t} \int \frac{\rho_{0}^{\beta+1}}{\rho}\left|\frac{\sigma}{\rho_{0}}\right|^{2} d x$

$$
\leq \frac{C}{\eta} \int 16 \pi^{2} \rho\left|\partial_{x}\left(r^{2} v\right)\right|^{2} d x+\eta \int \rho \rho_{0}^{2 \beta}\left|\frac{\sigma}{\rho_{0}}\right|^{2} d x-\frac{1}{2} \int \frac{\rho_{0}^{\beta+1}}{\rho^{2}} \partial_{t} \sigma\left|\frac{\sigma}{\rho_{0}}\right|^{2} d x
$$

which means that

$$
\frac{d}{d t} \mathscr{E}_{\beta}^{0, \sigma} \leq \frac{C}{\eta} \mathscr{D}^{0}+\eta_{\mathscr{E}}^{\mathscr{C}}{ }_{2 \beta+1}^{0, \sigma}+C \theta_{1} \mathscr{E}_{\beta}^{0, \sigma}
$$

Next we consider $\mathscr{E}^{1}$ and $\mathscr{D}^{1}$.

Lemma 4.2. We have

$$
\frac{d}{d t} \mathscr{E}^{1}+\mathscr{D}^{1} \leq\left(\eta+C \theta_{1}\right)^{\mathscr{E}} 1+\frac{1}{2} \mathscr{D}^{1}+C\left(\mathscr{E}^{0}+\mathscr{E}_{0}^{0, \sigma}\right)+q_{\mathscr{E}}^{2, v},
$$

where

$$
q:=q_{1}+q_{2}:=\left\|16 \pi^{2}\left(K \gamma+\frac{4}{K \gamma} a_{*}^{2}\left(\frac{\sigma}{\rho_{0}}\right)^{2}\right) \rho_{0}^{\gamma}\right\|_{L^{\infty}}+\left\|\frac{\left(1+\frac{\sigma}{\rho_{0}}\right)}{\eta\left(\delta+\frac{4 \varepsilon}{3}\right)}\right\|_{L^{\infty}}
$$

is bounded due to (4-20).

Proof. We divide the proof into steps.

Step $1\left(\mathscr{E}^{1, v}\right.$ and $\left.\mathscr{D}^{1, v}\right)$. Multiply (4-7) by $\partial_{t} v$ and integrate to get

$$
\int\left|\partial_{t} v\right|^{2} d x \underbrace{+\int 4 \pi r^{2} \partial_{t} v \partial_{x}\left\{K \gamma \rho_{0}^{\gamma} \frac{\sigma}{\rho_{0}}+a_{*} \rho_{0}^{\gamma}\left(\frac{\sigma}{\rho_{0}}\right)^{2}\right\} d x}_{(\mathrm{iv})}+\underbrace{\int \partial_{t} v \frac{x\left(r_{0}^{4}-r^{4}\right)}{r^{2} r_{0}^{4}} d x}_{(\mathrm{v})}-\underbrace{\int \partial_{t} v \mathcal{V} d x}_{(\mathrm{vi})}=0
$$

For (iv), we first expand 


$$
\begin{aligned}
\text { (iv) }= & \int 4 \pi r^{2} \partial_{t} v\left\{K \gamma \partial_{x}\left(\rho_{0}^{\gamma}\right) \frac{\sigma}{\rho_{0}}+K \gamma \rho_{0}^{\gamma} \partial_{x}\left(\frac{\sigma}{\rho_{0}}\right)\right\} d x \\
& +\int 4 \pi r^{2} \partial_{t} v\left\{\partial_{x}\left(a_{*} \rho_{0}^{\gamma}\right)\left(\frac{\sigma}{\rho_{0}}\right)^{2}+2 a_{*} \rho_{0}^{\gamma} \frac{\sigma}{\rho_{0}} \partial_{x}\left(\frac{\sigma}{\rho_{0}}\right)\right\} d x \\
= & :(i v)_{1}+(i v)_{2}+(\text { iv })_{3}+(i v)_{4},
\end{aligned}
$$

and then estimate

$$
\left|(\mathrm{iv})_{1}+(\mathrm{iv})_{3}\right| \leq \frac{1}{4} \int\left|\partial_{t} v\right|^{2} d x+C\left(1+\theta_{1}^{2}\right) \int\left|\frac{\sigma}{\rho_{0}}\right|^{2} d x \leq \frac{1}{4} \mathscr{D}^{1, v}+C_{0}^{\mathrm{c}} 0, \sigma
$$

and

$$
\begin{aligned}
\left|(\mathrm{iv})_{2}+(\mathrm{iv})_{4}\right| & \leq \int 8 \pi^{2} K \gamma r^{4} \rho_{0}^{\gamma}\left|\partial_{x}\left(\frac{\sigma}{\rho_{0}}\right)\right|^{2} d x+\int 8 \pi^{2}\left(K \gamma+\frac{4}{K \gamma} a_{*}^{2}\left(\frac{\sigma}{\rho_{0}}\right)^{2}\right) \rho_{0}^{\gamma}\left|\partial_{t} v\right|^{2} d x \\
& \leq \frac{1}{2} \mathscr{D}^{1, \sigma}+q_{1} \mathscr{E}^{2, v} .
\end{aligned}
$$

For (v), we get

$$
|(\mathrm{v})| \leq \frac{1}{4} \int\left|\partial_{t} v\right|^{2} d x+\int\left|\frac{x}{r^{2} r_{0}^{4}}\left(r^{2}+r_{0}^{2}\right)\left(r+r_{0}\right)\right|^{2}\left|r-r_{0}\right|^{2} d x \leq \frac{1}{4} \mathscr{D}^{1, v}+C_{\mathscr{E}}^{\mathscr{C}^{0}, r} .
$$

The term (vi) forms the energy $\mathscr{E}^{1, v}$ and nonlinear commutators:

$$
\begin{aligned}
(\mathrm{vi})=-\frac{1}{2} \frac{d}{d t}[ & \left.\delta \int 16 \pi^{2} \rho\left|\partial_{x}\left(r^{2} v\right)\right|^{2} d x+\frac{4 \varepsilon}{3} \int 16 \pi^{2} \rho r^{6}\left|\partial_{x}\left(\frac{v}{r}\right)\right|^{2} d x\right] \\
& +\frac{1}{2}\left[\delta \int 16 \pi^{2} \partial_{t} \sigma\left|\partial_{x}\left(r^{2} v\right)\right|^{2} d x+\frac{4 \varepsilon}{3} \int 16 \pi^{2} \partial_{t}\left(\rho r^{6}\right)\left|\partial_{x}\left(\frac{v}{r}\right)\right|^{2} d x\right] \\
& +\delta \int 16 \pi^{2} \partial_{x}(v \cdot 2 r v) \rho \partial_{x}\left(r^{2} v\right) d x+\frac{4 \varepsilon}{3} \int 16 \pi^{2} \rho r^{6} \partial_{x}\left(v \cdot\left(-\frac{v}{r^{2}}\right)\right) \partial_{x}\left(\frac{v}{r}\right) d x .
\end{aligned}
$$

Using (4-20) and the fact that

$$
\partial_{x}(v \cdot 2 r v)=2\left(\frac{v}{r} \partial_{x}\left(r^{2} v\right)+r^{2} v \partial_{x}\left(\frac{v}{r}\right)\right)
$$

and

$$
\partial_{x}\left(v \cdot\left(-\frac{v}{r^{2}}\right)\right) \partial_{x}\left(\frac{v}{r}\right)=-2 \frac{v}{r}\left|\partial_{x}\left(\frac{v}{r}\right)\right|^{2}
$$

the absolute values of the second and third lines may be bounded by $C \theta_{1}^{\mathscr{E}}{ }^{1, v}$.

We may now combine the above to deduce that

$$
\frac{d}{d t} \mathscr{E}^{1, v}+\mathscr{D}^{1, v} \leq \frac{1}{2} \mathscr{D}^{1}+C \theta_{1}^{\mathscr{E}^{1, v}}+C^{\mathscr{E}} \mathscr{E}^{0, r}+C_{\mathscr{E}_{0}^{0, \sigma}}^{\mathscr{C}}+q_{1} \mathscr{E}^{2, v}
$$


Step $2\left(\mathscr{C}^{1, \sigma}\right.$ and $\left.\mathscr{D}^{1, \sigma}\right)$. For the estimate of $\partial_{x}\left(\sigma / \rho_{0}\right)$, we first rewrite (4-7) by replacing $\rho \partial_{x}\left(r^{2} v\right)$ in $\mathscr{V}$ by $\partial_{t}\left(\sigma / \rho_{0}\right)$ through the continuity equation (4-4):

$$
\begin{aligned}
\left(\delta+\frac{4 \varepsilon}{3}\right) 4 \pi r^{2}\left\{\frac{\rho_{0}}{\rho} \partial_{t} \partial_{x}\left(\frac{\sigma}{\rho_{0}}\right)+\partial_{x}\left(\frac{\rho_{0}}{\rho}\right) \partial_{t}\left(\frac{\sigma}{\rho_{0}}\right)\right\}+\partial_{t} v+\frac{x\left(r_{0}^{4}-r^{4}\right)}{r^{2} r_{0}^{4}} \\
+4 \pi r^{2}\left\{K \gamma \rho_{0}^{\gamma} \partial_{x}\left(\frac{\sigma}{\rho_{0}}\right)+K \gamma \partial_{x}\left(\rho_{0}^{\gamma}\right) \frac{\sigma}{\rho_{0}}+\partial_{x}\left(a_{*} \rho_{0}^{\gamma}\right)\left(\frac{\sigma}{\rho_{0}}\right)^{2}+2 a_{*} \rho_{0}^{\gamma} \frac{\sigma}{\rho_{0}} \partial_{x}\left(\frac{\sigma}{\rho_{0}}\right)\right\}=0 .
\end{aligned}
$$

Note that

$$
\partial_{x}\left(\frac{\rho_{0}}{\rho}\right)=-\left(1+\frac{\sigma}{\rho_{0}}\right)^{-2} \partial_{x}\left(\frac{\sigma}{\rho_{0}}\right) .
$$

Multiplying (4-45) by $4 \pi r^{2} \partial_{x}\left(\frac{\sigma}{\rho_{0}}\right)$ and integrating, we are led to the estimate

$$
\begin{aligned}
\frac{1}{2} \frac{d}{d t} \int\left(\delta+\frac{4 \varepsilon}{3}\right) 16 \pi^{2} r^{4} \frac{1}{1+\frac{\sigma}{\rho_{0}}}\left|\partial_{x}\left(\frac{\sigma}{\rho_{0}}\right)\right|^{2} d x+\int 16 \pi^{2} K \gamma r^{4} \rho_{0}^{\gamma}\left|\partial_{x}\left(\frac{\sigma}{\rho_{0}}\right)\right|^{2} d x \\
\leq\left(\eta+C \theta_{1}\right)^{\mathscr{E}} \mathscr{E}^{1, \sigma}+C\left(\mathscr{E}^{\mathscr{E} 0, r}+\mathscr{\mathscr { E }}_{0}^{0, \sigma}\right)+\int \frac{1+\frac{\sigma}{\rho_{0}}}{2 \eta\left(\delta+\frac{4 \varepsilon}{3}\right)}\left|\partial_{t} v\right|^{2} d x .
\end{aligned}
$$

Note that the last term in (4-46) may be bounded by $q_{2} \mathscr{E}^{2, v}$. We then obtain (4-35) by combining (4-44) and (4-46).

The estimate (4-35) is not of a closed form by itself. Its use will be apparent when it is coupled with the result of the following lemma.

Lemma 4.3. $\quad \frac{d}{d t} \mathscr{E}^{2}+\mathscr{D}^{2} \leq\left(\eta+C \theta_{1}\right) \mathscr{\mathscr { C }}^{2}+C \theta_{1} \mathscr{D}^{0}+\left(\frac{1}{4}+C \theta_{1}\right) \mathscr{D}^{2}+C\left(\theta_{1} \mathscr{\mathscr { E }}_{0}^{0, \sigma}+\mathscr{E}^{0}+\mathscr{E}^{1}\right)$.

Proof. We take $\partial_{t}$ of (4-7) to see that

$$
\begin{aligned}
& \partial_{t}^{2} v+ 4 \pi r^{2} \partial_{x}\left\{K \gamma \rho_{0}^{\gamma} \partial_{t}\left(\frac{\sigma}{\rho_{0}}\right)+2 a_{*} \rho_{0}^{\gamma} \frac{\sigma}{\rho_{0}} \partial_{t}\left(\frac{\sigma}{\rho_{0}}\right)+\partial_{t} a_{*} \rho_{0}^{\gamma}\left(\frac{\sigma}{\rho_{0}}\right)^{2}\right\} \\
&+8 \pi r v\left\{K \gamma \rho_{0}^{\gamma} \partial_{x}\left(\frac{\sigma}{\rho_{0}}\right)+K \gamma \partial_{x}\left(\rho_{0}^{\gamma}\right) \frac{\sigma}{\rho_{0}}+\partial_{x}\left(a_{*} \rho_{0}^{\gamma}\right)\left(\frac{\sigma}{\rho_{0}}\right)^{2}+2 a_{*} \rho_{0}^{\gamma} \frac{\sigma}{\rho_{0}} \partial_{x}\left(\frac{\sigma}{\rho_{0}}\right)\right\} \\
&-2 \frac{x v\left(r_{0}^{4}-r^{4}\right)}{r^{3} r_{0}^{4}}-4 \frac{x r v}{r_{0}^{4}}=\partial_{t} \mathcal{} v
\end{aligned}
$$

The energy estimate (4-47) may be derived from (4-48) as in Lemma 4.1: we multiply (4-48) by $\partial_{t} v$, integrate over $x \in[0, M]$, and integrate various terms by parts in order to identify $d \mathscr{C}^{2} / d t, \mathscr{D}^{2}$, and some error (lower-order or commutator) terms, the latter of which may be estimated by the right side of (4-47). Since the argument is essentially the same as that of Lemma 4.1, we present only a sketch.

The product of $\partial_{t} v$ with the first two terms in the first line in (4-48) forms the energy term $\partial_{t} \mathscr{E}^{2}$ and 
some error terms:

$$
\int \partial_{t} v\left[\partial_{t}^{2} v+4 \pi r^{2} \partial_{x}\left(K \gamma \rho_{0}^{\gamma} \partial_{t}\left(\frac{\sigma}{\rho_{0}}\right)\right)\right] d x=\frac{1}{2} \frac{d}{d t}\left\{\int\left|\partial_{t} v\right|^{2} d x+\int \frac{K \gamma \rho_{0}^{\gamma-1}}{\left(1+\frac{\sigma}{\rho_{0}}\right)^{2}}\left|\partial_{t}\left(\frac{\sigma}{\rho_{0}}\right)\right|^{2} d x\right\}+\mathscr{L},
$$

where $\mathscr{E}$ is a term whose absolute value may be estimated by the right side of (4-47). Here we have used the continuity equation (4-4) and an integration by parts on the second term.

Next, we compute

$$
\partial_{t} \mathscr{V}=16 \pi^{2} r^{2} \partial_{x} \partial_{t} \mathcal{W}+16 \pi^{2}(2 r v) \partial_{x} \mathcal{W}+\frac{4 \varepsilon}{3} 12 \pi r^{2} \partial_{x}\left(\frac{\partial_{t} v}{r}\right)
$$

and note that the boundary condition $\mathcal{W}(M, t)=0$ implies that $\partial_{t} \mathcal{W}(M, t)=0$ as well. This allows us to integrate by parts without introducing boundary terms:

$$
\int 16 \pi^{2} r^{2} \partial_{x} \partial_{t} \mathcal{W} \partial_{t} v d x=-\int 16 \pi^{2}\left(r^{2} \partial_{t} v\right) \partial_{t}{ }^{W} d x
$$

Using this, we find that

$$
\int \partial_{t} \mathscr{V} \partial_{t} v d x=-\delta \int 16 \pi^{2} \rho\left|\partial_{x}\left(r^{2} \partial_{t} v\right)\right|^{2} d x+\frac{4 \varepsilon}{3} \int 16 \pi^{2} \rho r^{6}\left|\partial_{x}\left(\frac{\partial_{t} v}{r}\right)\right|^{2} d x+\mathscr{L},
$$

where again $\mathscr{E}$ is an error term with the property that $|\mathscr{E}|$ is bounded by the right side of (4-47).

Finally, all of the remaining terms that arise when we multiply (4-48) by $\partial_{t} v$ can also be estimated by the right side of (4-47). For example, the second term in the third line can be estimated by noting that $x r / r_{0}^{4}$ is bounded, which means that

$$
-\int 4 \frac{x r v}{r_{0}^{4}} \partial_{t} v d x \leq \eta \int\left|\partial_{t} v\right|^{2} d x+C \int|v|^{2} d x \leq \eta \mathscr{E}^{2}+C^{\mathscr{E}} 0
$$

Combining all of this, we find that

$$
\begin{aligned}
& \frac{1}{2} \frac{d}{d t}\left\{\int\left|\partial_{t} v\right|^{2} d x\right.\left.+\int \frac{K \gamma \rho_{0}^{\gamma-1}}{\left(1+\frac{\sigma}{\rho_{0}}\right)^{2}}\left|\partial_{t}\left(\frac{\sigma}{\rho_{0}}\right)\right|^{2} d x\right\} \\
&+\delta \int 16 \pi^{2} \rho\left|\partial_{x}\left(r^{2} \partial_{t} v\right)\right|^{2} d x+\frac{4 \varepsilon}{3} \int 16 \pi^{2} \rho r^{6}\left|\partial_{x}\left(\frac{\partial_{t} v}{r}\right)\right|^{2} d x \\
& \leq\left(\frac{1}{4}+C \theta_{1}\right) \mathscr{D}^{2}+\eta \mathscr{E}^{2}+C \theta_{1}\left(\mathscr{E}^{2}+\mathscr{D}^{0}\right)+C \theta_{1} \mathscr{E}_{0}^{0, \sigma}+C\left(\mathscr{E}^{0}+\mathscr{E}^{1}\right),
\end{aligned}
$$

which yields (4-47).

We now derive bootstrapped estimates for $\partial_{t}\left(\frac{\sigma}{\rho_{0}}\right)$. We take $\partial_{t}$ of (4-4) to get

$$
\frac{\rho_{0}}{\rho} \partial_{t}^{2}\left(\frac{\sigma}{\rho_{0}}\right)=-4 \pi \rho \partial_{x}\left(r^{2} \partial_{t} v\right)-8 \pi \rho \partial_{x}\left(r v^{2}\right)-4 \pi \partial_{t} \sigma \partial_{x}\left(r^{2} v\right)+\frac{\rho_{0}^{2}}{\rho^{2}}\left(\partial_{t}\left(\frac{\sigma}{\rho_{0}}\right)\right)^{2} .
$$

Next, we multiply (4-54) by $\rho_{0}^{\beta} \partial_{t}\left(\frac{\sigma}{\rho_{0}}\right)$ and integrate to see that 


$$
\begin{aligned}
& \frac{1}{2} \frac{d}{d t} \int \frac{\rho_{0}^{\beta+1}}{\rho}\left|\partial_{t}\left(\frac{\sigma}{\rho_{0}}\right)\right|^{2} d x \leq \frac{C}{\eta} \int 16 \pi^{2} \rho\left|\partial_{x}\left(r^{2} \partial_{t} v\right)\right|^{2} d x+\eta \int \rho \rho_{0}^{2 \beta}\left|\partial_{t}\left(\frac{\sigma}{\rho_{0}}\right)\right|^{2} d x \\
&-\int 8 \pi \frac{v}{r}\left\{\rho \partial_{x}\left(r^{2} v\right)+\rho r^{3} \partial_{x}\left(\frac{v}{r}\right)\right\} \rho_{0}^{\beta} \partial_{t}\left(\frac{\sigma}{\rho_{0}}\right) d x+ \frac{3}{2} \int \frac{\rho_{0}^{\beta+2}}{\rho^{2}}\left(\partial_{t}\left(\frac{\sigma}{\rho_{0}}\right)\right)^{3} d x \\
&-4 \pi \int \partial_{t} \sigma \partial_{x}\left(r^{2} v\right) \rho_{0}^{\beta} \partial_{t}\left(\frac{\sigma}{\rho_{0}}\right) d x .
\end{aligned}
$$

Then we estimate

$$
-\int 8 \pi \frac{v}{r}\left\{\rho \partial_{x}\left(r^{2} v\right)+\rho r^{3} \partial_{x}\left(\frac{v}{r}\right)\right\} \rho_{0}^{\beta} \partial_{t}\left(\frac{\sigma}{\rho_{0}}\right) d x-4 \pi \int \partial_{t} \sigma \partial_{x}\left(r^{2} v\right) \rho_{0}^{\beta} \partial_{t}\left(\frac{\sigma}{\rho_{0}}\right) d x \leq C \theta_{1} \mathscr{D}^{0}+C \theta_{1} \mathscr{E}_{2 \beta+1}^{2, \sigma}
$$

to obtain

$$
\frac{d}{d t} \mathscr{E}_{\beta}^{2, \sigma} \leq \frac{1}{4} \mathscr{D}^{2}+C \theta_{1} \mathscr{D}^{0}+\left(\eta+C \theta_{1}\right)_{2 \beta+1}^{\mathscr{C}} 2, \sigma=C \theta_{1} \mathscr{E}_{\beta}^{2, \sigma}
$$

Next we estimate $\mathscr{E}^{3}$ and $\mathscr{D}^{3}$.

Lemma 4.4. There exists an energy $\mathscr{F}^{3}$ such that

$$
\frac{d}{d t}\left[\mathscr{E}^{3}+\mathscr{F}^{3}\right]+\mathscr{D}^{3} \leq C \theta_{1} \mathscr{E}^{3}+\left(\frac{3}{8}+\frac{\theta_{1}}{4}\right) \mathscr{D}^{3}+C\left(\mathscr{E}_{0}^{2, \sigma}+\mathscr{E}^{2}+\mathscr{E}^{1}+\mathscr{E}^{0}\right) .
$$

Moreover, we have the estimate $\left|\mathscr{F}^{3}\right| \leq C \theta_{1}\left(\mathscr{E}^{3}+\mathscr{E}^{1}\right)$.

Proof. First recall (4-48) and rewrite it as

$$
\begin{aligned}
\partial_{t}^{2} v & +\underbrace{4 \pi r^{2}\left\{K \gamma \rho_{0}^{\gamma} \partial_{t} \partial_{x}\left(\frac{\sigma}{\rho_{0}}\right)+K \gamma \partial_{x}\left(\rho_{0}^{\gamma}\right) \partial_{t}\left(\frac{\sigma}{\rho_{0}}\right)\right\}}_{\left(\mathrm{a}_{1}\right)} \\
& +\underbrace{4 \pi r^{2}\left\{\partial_{t}\left[\partial_{x}\left(a_{*} \rho_{0}^{\gamma}\right)\left(\frac{\sigma}{\rho_{0}}\right)^{2}+2\left(a_{*} \rho_{0}^{\gamma}\right) \frac{\sigma}{\rho_{0}} \partial_{x}\left(\frac{\sigma}{\rho_{0}}\right)\right]\right\}}
\end{aligned}
$$

$$
+\underbrace{8 \pi r v\left\{K \gamma \rho_{0}^{\gamma} \partial_{x}\left(\frac{\sigma}{\rho_{0}}\right)+K \gamma \partial_{x}\left(\rho_{0}^{\gamma}\right) \frac{\sigma}{\rho_{0}}+\partial_{x}\left(a_{*} \rho_{0}^{\gamma}\right)\left(\frac{\sigma}{\rho_{0}}\right)^{2}+2 a_{*} \rho_{0}^{\gamma} \frac{\sigma}{\rho_{0}} \partial_{x}\left(\frac{\sigma}{\rho_{0}}\right)\right\}}
$$

$$
\underbrace{-2 \frac{x v\left(r_{0}^{4}-r^{4}\right)}{r^{3} r_{0}^{4}}-4 \frac{x r v}{r_{0}^{4}}}=\partial_{t} \mathscr{V},
$$

(c)

where $\partial_{t} \mathscr{V}$ is given in (4-49). To derive (4-57), we will multiply by $\partial_{t}^{2} v$ and integrate over $x$. We divide the estimates into the following steps. 
Step 1 We begin with an estimate of the product of $\partial_{t}^{2} v$ with the terms $\left(\mathrm{a}_{1}\right),\left(\mathrm{a}_{2}\right),(\mathrm{b})$, and (c). First, we use (4-45) to replace $\partial_{t} \partial_{x}\left(\sigma / \rho_{0}\right)$ by lower-order terms:

$$
\begin{aligned}
& \left(\mathrm{a}_{1}\right)+\left(\mathrm{a}_{2}\right)=4 \pi r^{2}\left\{K \gamma \rho_{0}^{\gamma} \partial_{t} \partial_{x}\left(\frac{\sigma}{\rho_{0}}\right)+K \gamma \partial_{x}\left(\rho_{0}^{\gamma}\right) \partial_{t}\left(\frac{\sigma}{\rho_{0}}\right)\right\} \\
& +4 \pi r^{2}\left\{\partial_{t}\left[\partial_{x}\left(a_{*} \rho_{0}^{\gamma}\right)\left(\frac{\sigma}{\rho_{0}}\right)^{2}+2\left(a_{*} \rho_{0}^{\gamma}\right) \frac{\sigma}{\rho_{0}} \partial_{x}\left(\frac{\sigma}{\rho_{0}}\right)\right]\right\} \\
& =-4 \pi r^{2}\left(K \gamma+2 a_{*} \frac{\sigma}{\rho_{0}}\right) \rho_{0}^{\gamma} \frac{\rho}{\rho_{0}} \partial_{x}\left(\frac{\rho_{0}}{\rho}\right) \partial_{t}\left(\frac{\sigma}{\rho_{0}}\right) \\
& -\left(K \gamma+2 a_{*} \frac{\sigma}{\rho_{0}}\right) \rho_{0}^{\gamma} \frac{\rho}{\rho_{0}\left(\delta+\frac{4 \varepsilon}{3}\right)}\left\{\partial_{t} v+\frac{x\left(r_{0}^{4}-r^{4}\right)}{r^{2} r_{0}^{4}}+4 \pi r^{2}\left[K \gamma \rho_{0}^{\gamma} \partial_{x}\left(\frac{\sigma}{\rho_{0}}\right)\right.\right. \\
& \left.\left.+K \gamma \partial_{x}\left(\rho_{0}^{\gamma}\right) \frac{\sigma}{\rho_{0}}+\partial_{x}\left(a_{*} \rho_{0}^{\gamma}\right)\left(\frac{\sigma}{\rho_{0}}\right)^{2}+2 a_{*} \rho_{0}^{\gamma} \frac{\sigma}{\rho_{0}} \partial_{x}\left(\frac{\sigma}{\rho_{0}}\right)\right]\right\} \\
& +4 \pi r^{2}\left\{K \gamma \partial_{x}\left(\rho_{0}^{\gamma}\right) \partial_{t}\left(\frac{\sigma}{\rho_{0}}\right)+\partial_{t}\left[\partial_{x}\left(a_{*} \rho_{0}^{\gamma}\right)\left(\frac{\sigma}{\rho_{0}}\right)^{2}\right]+2 \rho_{0}^{\gamma} \partial_{t}\left(a_{*} \frac{\sigma}{\rho_{0}}\right) \partial_{x}\left(\frac{\sigma}{\rho_{0}}\right)\right\} \\
& =:\left(A_{1}\right)+\left(A_{2}\right)+\left(A_{3}\right) \text {. }
\end{aligned}
$$

Then $\int \partial_{t}^{2} v \cdot\left[\left(\mathrm{a}_{1}\right)+\left(\mathrm{a}_{2}\right)\right] d x$ can be estimated as follows:

$$
\begin{aligned}
& \int \partial_{t}^{2} v \cdot\left(\mathrm{A}_{1}\right) d x \leq \frac{\theta_{1}}{8} \int\left|\partial_{t}^{2} v\right|^{2} d x+C \theta_{1} \int \frac{\rho_{0}^{2 \gamma+2}}{\rho^{2}} r^{4}\left|\partial_{x}\left(\frac{\sigma}{\rho_{0}}\right)\right|^{2} d x \leq \frac{\theta_{1}}{8} \mathscr{D}^{3}+C \theta_{1} \mathscr{E}, \sigma \\
& \int \partial_{t}^{2} v \cdot\left(\mathrm{A}_{2}\right) d x \leq \frac{3}{32} \int\left|\partial_{t}^{2} v\right|^{2} d x+C\left[\mathscr{E}^{2, v}+\mathscr{E}^{0, r}+\left(1+\theta_{1}\right)\left(\mathscr{E}^{1, \sigma}+\mathscr{E}^{0, \sigma}\right)\right], \\
& \int \partial_{t}^{2} v \cdot\left(\mathrm{A}_{3}\right) d x \leq \frac{3}{32} \int\left|\partial_{t}^{2} v\right|^{2} d x+C \theta_{1} \mathscr{E}^{1, \sigma}+C\left(1+\theta_{1}\right)_{\mathscr{E}} \mathscr{E}_{0}^{2, \sigma} .
\end{aligned}
$$

For (b) and (c), we may estimate

$$
\begin{aligned}
& \int \partial_{t}^{2} v \cdot(\mathrm{b}) d x \leq \frac{\theta_{1}}{8} \int\left|\partial_{t}^{2} v\right|^{2} d x+C \theta_{1}\left(\mathscr{E}^{1, \sigma}+\mathscr{E}_{0}^{0, \sigma}\right), \\
& \int \partial_{t}^{2} v \cdot(\mathrm{c}) d x \leq \frac{3}{32} \int\left|\partial_{t}^{2} v\right|^{2} d x+C\left(\mathscr{E}^{0, v}+\mathscr{E}^{0, r}\right) .
\end{aligned}
$$

Combining the above, we arrive at an estimate for $\int \partial_{t}^{2} v \cdot\left[\left(\mathrm{a}_{1}\right)+\left(\mathrm{a}_{2}\right)+(\mathrm{b})+(\mathrm{c})\right] d x$.

Step 2 (the viscosity term). Now we consider the viscosity term, $\partial_{t} \mathscr{V}$. We claim that there exist $\mathscr{F}^{3}, G$ such that

$$
\int \partial_{t}^{2} v \cdot \partial_{t} \mathscr{V} d x=-\frac{d}{d t} \mathscr{E}^{3}-\frac{d}{d t} \mathscr{F}^{3}+G,
$$


where

$$
\left|\mathscr{F}^{3}\right| \leq C \theta_{1}\left(\mathscr{E}^{3}+\mathscr{E}^{1}\right) \quad \text { and } \quad|G| \leq \frac{3}{32} \mathscr{D}^{3}+C \theta_{1}\left(\mathscr{E}^{3}+\mathscr{E}_{0}^{2, \sigma}+\mathscr{E}^{1}\right) .
$$

Recall that $\partial_{t} \mathscr{V}$ may be computed as in (4-49), and that $\partial_{t} \mathscr{W}(M, t)=0$. Then a simple but lengthy computation, using integration by parts, reveals that

$\int \partial_{t}^{2} v \cdot \partial_{t} \mathcal{V} d x=-\frac{1}{2} \frac{d}{d t} \int \delta 16 \pi^{2} \rho\left|\partial_{x}\left(r^{2} \partial_{t} v\right)\right|^{2} d x+\frac{4 \varepsilon}{3} 16 \pi^{2} \rho r^{6}\left|\partial_{x}\left(\frac{\partial_{t} v}{r}\right)\right|^{2} d x+G_{0}+Y$,

where

$$
\begin{aligned}
G_{0}=\int\left(\frac{\partial_{t} \sigma}{2 \rho}+\frac{2 v}{r}\right)\{\delta & \left.16 \pi^{2} \rho\left|\partial_{x}\left(r^{2} \partial_{t} v\right)\right|^{2}+\frac{4 \varepsilon}{3} 16 \pi^{2} \rho r^{6}\left|\partial_{x}\left(\frac{\partial_{t} v}{r}\right)\right|^{2}\right\} d x \\
& +\int \rho r^{3} \partial_{x}\left(\frac{v}{r}\right)\left\{\delta 32 \pi^{2} \frac{\partial_{t} v}{r} \partial_{x}\left(r^{2} \partial_{t} v\right)-\frac{4 \varepsilon}{3} 16 \pi^{2} \frac{\partial_{t} v}{r} r^{3} \partial_{x}\left(\frac{\partial_{t} v}{r}\right)\right\} d x
\end{aligned}
$$

and $Y=Y_{1}+Y_{2}$ with

$$
\begin{aligned}
& Y_{1}=- 16 \pi^{2} \int\left[\delta \partial_{t} \sigma \partial_{x}\left(r^{2} v\right)+\delta \rho \partial_{x}\left(2 r v^{2}\right)\right] \partial_{x}\left(r^{2} \partial_{t}^{2} v\right) d x \\
&-16 \pi^{2} \int\left[\frac{4 \varepsilon}{3} \partial_{t}\left(\rho r^{3}\right) \partial_{x}\left(\frac{v}{r}\right)-\frac{4 \varepsilon}{3} \rho r^{3} \partial_{x}\left(\frac{v^{2}}{r^{2}}\right)\right] \partial_{x}\left(r^{2} \partial_{t}^{2} v\right) d x, \\
& Y_{2}=32 \pi^{2} \int r v \partial_{t}^{2} v \partial_{x}\left[\delta \rho \partial_{x}\left(r^{2} v\right)+\frac{4 \varepsilon}{3} \rho r^{3} \partial_{x}\left(\frac{v}{r}\right)\right] d x .
\end{aligned}
$$
Let us define the quantity $Q$ such that $Y_{1}=-16 \pi^{2} \int Q \partial_{x}\left(r^{2} \partial_{t}^{2} v\right) d x$, that is, $Q$ is the sum of the bracketed
terms in the $Y_{1}$ integrand. Then we may compute

$$
Y_{1}=\frac{d}{d t} \int-16 \pi^{2} \partial_{x}\left(r^{2} \partial_{t} v\right) Q d x+\int 16 \pi^{2}\left(\partial_{x}\left(2 r v \partial_{t} v\right) Q+\partial_{x}\left(r^{2} \partial_{t} v\right) \partial_{t} Q\right) d x:=-\frac{d}{d t} \mathscr{F}_{1}^{3}+G_{1} .
$$

Similarly, we have that

$$
\begin{aligned}
Y_{2}=\frac{d}{d t} & \int-16 \pi^{2} \partial_{x}\left(r^{2} \partial_{t} v\right) \frac{2 v}{r}\left[\delta \rho \partial_{x}\left(r^{2} v\right)+\frac{4 \varepsilon}{3} \rho r^{3} \partial_{x}\left(\frac{v}{r}\right)\right] d x \\
& +16 \pi^{2} \int\left[\partial_{x}\left(2 r v \partial_{t} v\right) \frac{2 v}{r} \mathscr{W}+\partial_{x}\left(r^{2} \partial_{t} v\right) \partial_{t}\left(\frac{2 v}{r}\right) \mathcal{W}+\partial_{x}\left(r^{2} \partial_{t} v\right) \frac{2 v}{r} \partial_{t} \mathcal{W}\right] d x \\
& \quad-16 \pi^{2} \int r^{2} \partial_{t}^{2} v \mathcal{W} \partial_{x}\left(\frac{2 v}{r}\right) d x \\
=- & \frac{d}{d t} \mathscr{F}_{2}^{3}+G_{2} .
\end{aligned}
$$

Combining the above, we find that (4-64) holds with $\mathscr{F}^{3}=\mathscr{F}_{1}^{3}+\mathscr{F}_{2}^{3}$ and $G=G_{0}+G_{1}+G_{2}$. To complete the proof of the claim, we note that the estimates (4-65) follow from the definition of $\mathscr{F}^{3}$ and $G$, using (4-54) to replace $\partial_{t}^{2} \sigma$ by other terms. 
Step 3 (conclusion). The only term that remains is

$$
\int \partial_{t}^{2} v \partial_{t}^{2} v d x=\mathscr{D}^{3}
$$

With this, all of the terms in (4-58) are accounted for. We may then combine the analysis of Steps 1 and 2 to deduce the estimate (4-57).

We now bootstrap more estimates. First, we multiply (4-22) by $\frac{1}{\rho}\left(1-\frac{r_{0}}{r}\right)$ and integrate to get

$$
\frac{d}{d t} \mathscr{E}_{b}^{0, r} \leq\left(\eta+C \theta_{1}\right)^{\mathscr{E}} \mathscr{E}_{b}^{0, r}+C \mathscr{E}_{\mathscr{E}}^{\mathscr{1}}{ }^{1, v}
$$

where we have used (4-23) to control $\int \frac{|v|^{2}}{r^{2} \rho} d x \leq C_{\mathscr{E}}^{\mathscr{E} 1, v}$. By multiplying (4-45) by $\frac{1}{\rho_{0}} \partial_{x}\left(\frac{\sigma}{\rho_{0}}\right)$ and integrating, we get

$$
\frac{d}{d t} \mathscr{E}_{b}^{1, \sigma}+\mathscr{D}_{b}^{1, \sigma} \leq\left(\eta+C \theta_{1}\right) \mathscr{E}_{b}^{1, \sigma}+C\left(\mathscr{E}^{3}+\mathscr{E}_{-1}^{0, \sigma}+\mathscr{E}_{b}^{0, r}\right)
$$

Note that here we have again used (4-23) to control $\int\left|\partial_{t} v\right|^{2} /\left(r^{2} \rho\right) d x$, which is possible since (4-23) is valid for any choice of $v$, not just solutions. From (4-45) we also see that

$$
\mathscr{E}_{a}^{3, \sigma}=\int\left(\delta+\frac{4 \varepsilon}{3}\right)^{2} 16 \pi^{2} \frac{r^{2}}{\rho}\left|\partial_{x} \partial_{t}\left(\frac{\sigma}{\rho_{0}}\right)\right|^{2} d x \leq C\left(\mathscr{E}^{3}+\mathscr{E}_{b}^{1, \sigma}+\mathscr{E}_{-1}^{0, \sigma}+\mathscr{E}^{0}\right) .
$$

Next, by applying $\partial_{x}$ to (4-4), we find that

$$
\frac{\rho_{0}}{\rho} \partial_{x} \partial_{t}\left(\frac{\sigma}{\rho_{0}}\right)+\partial_{x}\left(\frac{\rho_{0}}{\rho}\right) \partial_{t}\left(\frac{\sigma}{\rho_{0}}\right)+4 \pi \partial_{x}\left(\rho \partial_{x}\left(r^{2} v\right)\right)=0 .
$$

We then use this to get

$$
\mathscr{E}_{a_{1}}^{3, v}=\int \frac{r^{2}}{\rho}\left|\partial_{x}\left(\rho \partial_{x}\left(r^{2} v\right)\right)\right|^{2} d x \leq C\left(\mathscr{E}_{a}^{3, \sigma}+\mathscr{E}_{b}^{1, \sigma}\right)
$$

Since $\int \rho r^{6}\left|\partial_{x}\left(\frac{v}{r}\right)\right|^{2} d x \leq C \mathscr{E}^{1},(4-7)$ implies that

$$
\mathscr{E}_{a_{2}}^{3, v}=\int \rho r^{6}\left|\partial_{x}\left(\rho r^{3} \partial_{x}\left(\frac{v}{r}\right)\right)\right|^{2} d x \leq C\left(\mathscr{E}^{2}+\mathscr{E}^{1}+\mathscr{E}^{0}\right)
$$

We now illustrate how the higher-order energy estimates of spatial derivatives of $\partial_{x}\left(\sigma / \rho_{0}\right)$ and $\partial_{x}\left(\rho \partial_{x}\left(r^{2} v\right)\right)$ work. The following lemma concerns the estimate of $\partial_{x}\left(r^{4} \partial_{x}\left(\sigma / \rho_{0}\right)\right)$.

Lemma 4.5. $\quad \frac{d}{d t} \mathscr{E}^{4}+\mathscr{D}^{4} \leq\left(\eta+C \theta_{1}\right)_{\mathscr{E}^{4}}+C\left(\mathscr{E}^{3}+\mathscr{E}^{0, r}+\mathscr{E}_{b}^{0, r}+\mathscr{E}_{-1}^{0, \sigma}\right)+C \theta_{1}\left(\mathscr{E}_{b}^{1, \sigma}+\mathscr{E}_{a}^{3}, \sigma\right)$. 
Proof. First, we multiply (4-45) by $r^{2}$ and apply $\partial_{x}$ to get

$$
\left(\delta+\frac{4 \varepsilon}{3}\right) 4 \pi\{\underbrace{\frac{\rho_{0}}{\rho} \partial_{x}\left(r^{4} \partial_{t} \partial_{x}\left(\frac{\sigma}{\rho_{0}}\right)\right)}+2 \underbrace{2 \partial_{x}\left(\frac{\rho_{0}}{\rho}\right) r^{4} \partial_{t} \partial_{x}\left(\frac{\sigma}{\rho_{0}}\right)}+\underbrace{\partial_{x}\left(r^{4} \partial_{x}\left(\frac{\rho_{0}}{\rho}\right)\right) \partial_{t}\left(\frac{\sigma}{\rho_{0}}\right)}\}
$$

(iii)

$$
\begin{array}{ll}
+ & \underbrace{\partial_{x}\left(r^{2} \partial_{t} v\right)}_{(\mathrm{iv})}+\underbrace{\frac{\left(r_{0}^{4}-r^{4}\right)}{r_{0}^{4}}}_{(\mathrm{v})}-\underbrace{\frac{x r^{4}}{\pi r_{0}^{7} \rho}\left(1-\left(\frac{r_{0}}{r}\right)^{3}+\frac{\sigma}{\rho_{0}}\right)}_{(\mathrm{vi})}+4 \pi \underbrace{K \gamma \rho_{0}^{\gamma} \partial_{x}\left(r^{4} \partial_{x}\left(\frac{\sigma}{\rho_{0}}\right)\right)}_{(\mathrm{vii})} \\
+ & 4 \pi\left\{2 K \gamma r^{4} \partial_{x}\left(\rho_{0}^{\gamma}\right) \partial_{x}\left(\frac{\sigma}{\rho_{0}}\right)+K \gamma \partial_{x}\left(r^{4} \partial_{x}\left(\rho_{0}^{\gamma}\right)\right) \frac{\sigma}{\rho_{0}}\right\} \\
+ & 4 \pi\left\{2 r^{4} \partial_{x}\left(a_{*} \rho_{0}^{\gamma}\right) \frac{\sigma}{\rho_{0}} \partial_{x}\left(\frac{\sigma}{\rho_{0}}\right)+\partial_{x}\left(r^{4} \partial_{x}\left(a_{*} \rho_{0}^{\gamma}\right)\right)\left(\frac{\sigma}{\rho_{0}}\right)^{2}\right\} \\
+ & 4 \pi\left\{2 a_{*} \rho_{0}^{\gamma} \frac{\sigma}{\rho_{0}} \partial_{x}\left(r^{4} \partial_{x}\left(\frac{\sigma}{\rho_{0}}\right)\right)+2 r^{4} \partial_{x}\left(a_{*} \rho_{0}^{\gamma} \frac{\sigma}{\rho_{0}}\right) \partial_{x}\left(\frac{\sigma}{\rho_{0}}\right)\right\} \\
=0 . \quad &
\end{array}
$$

The energy inequality (4-78) can be derived as in Step 2 of Lemma 4.2 by multiplying (4-79) by $\rho \partial_{x}\left(r^{4} \partial_{x}\left(\sigma / \rho_{0}\right)\right)$ and integrating over $x$. We provide the details on how (i)-(vii) can be treated; other terms can be estimated similarly.

$$
\begin{aligned}
\int\left(\delta+\frac{4 \varepsilon}{3}\right) 4 \pi(\mathrm{i}) \cdot \rho \partial_{x}( & \left.r^{4} \partial_{x}\left(\frac{\sigma}{\rho_{0}}\right)\right) d x \\
=\frac{1}{2} \frac{d}{d t} \int\left(\delta+\frac{4 \varepsilon}{3}\right) 4 \pi \rho_{0}\left|\partial_{x}\left(r^{4} \partial_{x}\left(\frac{\sigma}{\rho_{0}}\right)\right)\right|^{2} d x & \quad\left(\delta+\frac{4 \varepsilon}{3}\right) 4 \pi \underbrace{\int \rho_{0} \partial_{x}\left(\partial_{t}\left(r^{4}\right) \partial_{x}\left(\frac{\sigma}{\rho_{0}}\right)\right) \partial_{x}\left(r^{4} \partial_{x}\left(\frac{\sigma}{\rho_{0}}\right)\right) d x}_{(*)} .
\end{aligned}
$$

Since $\partial_{t}\left(r^{4}\right)=4 \frac{v}{r} r^{4}$,

$$
(*)=\int 4 \frac{v}{r} \rho_{0}\left|\partial_{x}\left(r^{4} \partial_{x}\left(\frac{\sigma}{\rho_{0}}\right)\right)\right|^{2} d x+\int 4 \partial_{x}\left(\frac{v}{r}\right) r^{4} \partial_{x}\left(\frac{\sigma}{\rho_{0}}\right) \rho_{0} \partial_{x}\left(r^{4} \partial_{x}\left(\frac{\sigma}{\rho_{0}}\right)\right) d x
$$

and since $\left|\frac{v}{r}\right|$ and $\left|\rho r^{3} \partial_{x}\left(\frac{v}{r}\right)\right|$ are bounded by $\theta_{1}$,

$$
|(*)| \leq C \theta_{1}\left(\mathscr{E}^{4}+\mathscr{E}_{b}^{1, \sigma}\right) .
$$

For (ii), we write

$$
\int(\mathrm{ii}) \cdot \rho \partial_{x}\left(r^{4} \partial_{x}\left(\frac{\sigma}{\rho_{0}}\right)\right) d x=-\int \frac{\rho_{0} r^{3} \partial_{x}\left(\frac{\sigma}{\rho_{0}}\right)}{1+\frac{\sigma}{\rho_{0}}} \cdot \frac{r}{\sqrt{\rho}} \partial_{t} \partial_{x}\left(\frac{\sigma}{\rho_{0}}\right) \cdot \sqrt{\rho} \partial_{x}\left(r^{4} \partial_{x}\left(\frac{\sigma}{\rho_{0}}\right)\right) d x
$$


and therefore

$$
\left|\int(\mathrm{ii}) \cdot \rho \partial_{x}\left(r^{4} \partial_{x}\left(\frac{\sigma}{\rho_{0}}\right)\right) d x\right| \leq C \theta_{1}\left(\mathscr{E}_{a}^{3, \sigma}+\mathscr{E}^{4}\right)
$$

It is easy to see that

$$
\left|\int[(\mathrm{iii})+(\mathrm{iv})+(\mathrm{v})-(\mathrm{vi})] \cdot \rho \partial_{x}\left(r^{4} \partial_{x}\left(\frac{\sigma}{\rho_{0}}\right)\right) d x\right| \leq\left(\frac{\eta}{2}+C \theta_{1}\right) \mathscr{E}^{4}+C\left(\mathscr{E}^{3}+\mathscr{E}^{0, r}+\mathscr{E}_{b}^{0, r}+\mathscr{E}_{-1}^{0, \sigma}\right) .
$$

Finally, (vii) forms the dissipation $\mathscr{D}^{4}$.

We also get an estimate for $\partial_{x}\left(r^{4} \partial_{t} \partial_{x}\left(\frac{\sigma}{\rho_{0}}\right)\right)$ from (4-79):

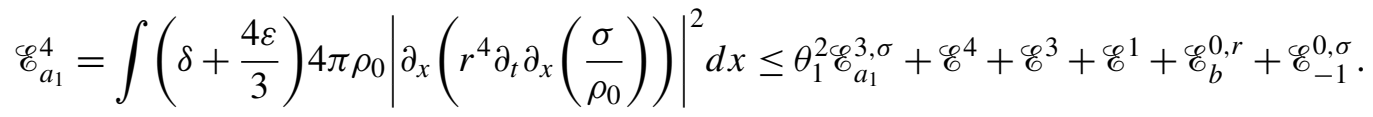

To derive an estimate of the third spatial derivatives of $v$, we first multiply (4-75) by $r^{4}$ and then apply $\partial_{x}$ :

$$
\begin{aligned}
\frac{\rho_{0}}{\rho} \partial_{x}\left(r^{4} \partial_{x} \partial_{t}\left(\frac{\sigma}{\rho_{0}}\right)\right)+2 r^{4} \partial_{x}\left(\frac{\rho_{0}}{\rho}\right) \partial_{x} \partial_{t}\left(\frac{\sigma}{\rho_{0}}\right)+\partial_{x}\left(r^{4} \partial_{x}\left(\frac{\rho_{0}}{\rho}\right)\right) & \partial_{t}\left(\frac{\sigma}{\rho_{0}}\right) \\
& +4 \pi \partial_{x}\left(r^{4} \partial_{x}\left(\rho \partial_{x}\left(r^{2} v\right)\right)\right)=0 .
\end{aligned}
$$

Thus, we obtain

$$
\mathscr{E}_{a_{2}}^{4}=\int 16 \pi^{2} \rho_{0}\left|\partial_{x}\left(r^{4} \partial_{x}\left(\rho \partial_{x}\left(r^{2} v\right)\right)\right)\right|^{2} d x \leq \mathscr{E}_{a_{1}}^{4}+\theta_{1}^{2}\left(\mathscr{E}_{a}^{3, \sigma}+\mathscr{E}^{4}\right)
$$

We now present the higher-order energy estimates. We start with $\mathscr{C}^{5}$ and $\mathscr{E}_{a}^{5}$.

\section{Lemma 4.6.}

$$
\frac{d}{d t} \mathscr{E}^{5}+\mathscr{D}^{5} \leq\left(\eta+C \theta_{1}\right)^{\mathscr{E}^{5}}+\left(\frac{1}{2}+C \theta_{1}\right) \mathscr{D}^{5}+C \theta_{1}^{2 \mathscr{E}^{3}}+C \theta_{1}\left(\mathscr{E}_{a}^{3, \sigma}+\mathscr{E}^{3}+\mathscr{E}^{2}+\mathscr{E}^{1}\right)+C \mathscr{E}^{2}
$$

Proof. We apply $\partial_{t}$ to (4-48) to see that

$$
\begin{aligned}
& \partial_{t}^{3} v+4 \pi r^{2} \partial_{x}\left\{\left(K \gamma+2 a_{*} \frac{\sigma}{\rho_{0}}\right) \rho_{0}^{\gamma} \partial_{t}^{2}\left(\frac{\sigma}{\rho_{0}}\right)+\rho_{0}^{\gamma}\left[a_{*}\left(\partial_{t}\left(\frac{\sigma}{\rho_{0}}\right)\right)^{2}+4 \partial_{t} a_{*} \frac{\sigma}{\rho_{0}} \partial_{t}\left(\frac{\sigma}{\rho_{0}}\right)+\partial_{t}^{2} a_{*}\left(\frac{\sigma}{\rho_{0}}\right)^{2}\right]\right\} \\
& +16 \pi v r\left\{K \gamma \rho_{0}^{\gamma} \partial_{t} \partial_{x}\left(\frac{\sigma}{\rho_{0}}\right)+K \gamma \partial_{x}\left(\rho_{0}^{\gamma}\right) \partial_{t}\left(\frac{\sigma}{\rho_{0}}\right)\right\}+16 \pi v r \partial_{t}\left[\partial_{x}\left(a_{*} \rho_{0}^{\gamma}\right)\left(\frac{\sigma}{\rho_{0}}\right)^{2}+2\left(a_{*} \rho_{0}^{\gamma}\right) \frac{\sigma}{\rho_{0}} \partial_{x}\left(\frac{\sigma}{\rho_{0}}\right)\right] \\
& \quad+8 \pi\left(r \partial_{t} v+v^{2}\right)\left\{K \gamma \rho_{0}^{\gamma} \partial_{x}\left(\frac{\sigma}{\rho_{0}}\right)+K \gamma \partial_{x}\left(\rho_{0}^{\gamma}\right) \frac{\sigma}{\rho_{0}}+\partial_{x}\left(a_{*} \rho_{0}^{\gamma}\right)\left(\frac{\sigma}{\rho_{0}}\right)^{2}+2 a_{*} \rho_{0}^{\gamma} \frac{\sigma}{\rho_{0}} \partial_{x}\left(\frac{\sigma}{\rho_{0}}\right)\right\} \\
& \quad-\frac{2 x\left(r_{0}^{4}-r^{4}\right) \partial_{t} v}{r^{3} r_{0}^{4}}-\frac{4 x r \partial_{t} v}{r_{0}^{4}}-\frac{12 x v^{2}}{r_{0}^{4}}+\frac{6 x\left(r_{0}^{4}-r^{4}\right) v^{2}}{r^{4} r_{0}^{4}} \\
& =\partial_{t}^{2} V .
\end{aligned}
$$


We derive the energy estimate of (4-89) from (4-48) by proceeding as in the proofs of Lemmas 4.1 and 4.3. That is, we multiply the resulting equation by $\partial_{t}^{2} v$ and integrate over $x$, integrating by parts in some terms to recover $d_{\mathscr{E}}^{\mathscr{C} 5} / d t, \mathscr{D}^{5}$, and some error terms that can be estimated by the right side of (4-89). Since the method of proof is already recorded in Lemmas 4.1 and 4.3 , we omit further details.

An estimate of $\partial_{x}\left(\rho \partial_{x}\left(\partial_{t}\left[r^{2} v\right]\right)\right)$ can be obtained through (4-48):

$$
\mathscr{E}_{a}^{5, v} \leq C\left(\mathscr{E}^{5}+\mathscr{E}_{a}^{3, \sigma}+\mathscr{E}^{1}\right)+C \theta_{1}\left(\mathscr{E}_{a_{1}^{3}}^{3, v}+\mathscr{E}_{a}^{3, \sigma}+\mathscr{E}^{1}+\mathscr{E}^{0}\right) .
$$

We now bootstrap to control $\partial_{t}^{2}\left(\frac{\sigma}{\rho_{0}}\right)$. We apply $\partial_{t}$ to (4-54) to get

$$
\partial_{t}^{3}\left(\frac{\sigma}{\rho_{0}}\right)=-4 \pi \frac{\rho}{\rho_{0}} \rho \partial_{x}\left(r^{2} \partial_{t}^{2} v\right)-24 \pi \frac{\rho}{\rho_{0}} \underbrace{\rho \partial_{x}\left(r v \partial_{t} v\right)}_{(\mathrm{a})}-8 \pi \frac{\rho}{\rho_{0}} \underbrace{\rho \partial_{x}\left(v^{3}\right)}_{(\mathrm{b})}+6 \frac{\partial_{t} \sigma}{\rho} \partial_{t}^{2}\left(\frac{\sigma}{\rho_{0}}\right)-6 \frac{\left(\partial_{t} \sigma\right)^{3}}{\rho_{0} \rho^{2}} .
$$

Note that

$$
\text { (a) }=\frac{v}{r} \rho \partial_{x}\left(r^{2} \partial_{t} v\right)+\rho r^{3} \partial_{x}\left(\frac{v}{r}\right) \frac{\partial_{t} v}{r}
$$

and

$$
\text { (b) }=3 r^{3} \rho\left(\frac{v}{r}\right)^{2} \partial_{x}\left(\frac{v}{r}\right)+\frac{3}{4 \pi}\left(\frac{v}{r}\right)^{3}
$$

and thus by multiplying (4-92) by $\frac{1}{\rho_{0}} \partial_{t}^{2}\left(\frac{\sigma}{\rho_{0}}\right)$ and integrating, we obtain

$$
\frac{d}{d t} \mathscr{E}_{-1}^{5, \sigma} \leq\left(\eta+C \theta_{1}\right)_{-1}^{\mathscr{E}_{-1}^{5, \sigma}}+C \mathscr{D}^{5}+C \theta_{1}^{2}\left(\mathscr{E}^{3}+\mathscr{E}^{1}+\mathscr{E}_{-1}^{2, \sigma}\right) .
$$

Next, we take $\partial_{t}$ of (4-45) to see that

$$
\begin{aligned}
\left(\delta+\frac{4 \varepsilon}{3}\right) 4 \pi r^{2} & \left\{\frac{\rho_{0}}{\rho} \partial_{t}^{2} \partial_{x}\left(\frac{\sigma}{\rho_{0}}\right)+\partial_{x}\left(\frac{\rho_{0}}{\rho}\right) \partial_{t}^{2}\left(\frac{\sigma}{\rho_{0}}\right)+\partial_{t}\left(\frac{\rho_{0}}{\rho}\right) \partial_{t} \partial_{x}\left(\frac{\sigma}{\rho_{0}}\right)+\partial_{t} \partial_{x}\left(\frac{\rho_{0}}{\rho}\right) \partial_{t}\left(\frac{\sigma}{\rho_{0}}\right)\right\} \\
+ & \left(\delta+\frac{4 \varepsilon}{3}\right) \frac{v}{r} 8 \pi r^{2}\left\{\frac{\rho_{0}}{\rho} \partial_{t} \partial_{x}\left(\frac{\sigma}{\rho_{0}}\right)+\partial_{x}\left(\frac{\rho_{0}}{\rho}\right) \partial_{t}\left(\frac{\sigma}{\rho_{0}}\right)\right\} \\
+ & \partial_{t}^{2} v-2 \frac{v}{r}\left(\frac{x}{r^{2}}+\frac{x r^{2}}{r_{0}^{4}}\right)+4 \pi r^{2}\left\{K \gamma \rho_{0}^{\gamma} \partial_{t} \partial_{x}\left(\frac{\sigma}{\rho_{0}}\right)+K \gamma \partial_{x}\left(\rho_{0}^{\gamma}\right) \partial_{t}\left(\frac{\sigma}{\rho_{0}}\right)\right\} \\
+ & 4 \pi r^{2} \partial_{t}\left[\partial_{x}\left(a_{*} \rho_{0}^{\gamma}\right)\left(\frac{\sigma}{\rho_{0}}\right)^{2}+2 a_{*} \rho_{0}^{\gamma} \frac{\sigma}{\rho_{0}} \partial_{x}\left(\frac{\sigma}{\rho_{0}}\right)\right] \\
+ & \frac{v}{r} 8 \pi r^{2}\left\{K \gamma \rho_{0}^{\gamma} \partial_{x}\left(\frac{\sigma}{\rho_{0}}\right)+K \gamma \partial_{x}\left(\rho_{0}^{\gamma}\right) \frac{\sigma}{\rho_{0}}+\partial_{x}\left(a_{*} \rho_{0}^{\gamma}\right)\left(\frac{\sigma}{\rho_{0}}\right)^{2}+2 a_{*} \rho_{0}^{\gamma} \frac{\sigma}{\rho_{0}} \partial_{x}\left(\frac{\sigma}{\rho_{0}}\right)\right\}
\end{aligned}
$$

$=0$. 
Therefore, by squaring (4-94) and integrating, we find that

$$
\int\left(\delta+\frac{4 \varepsilon}{3}\right)^{2} 16 \pi^{2} r^{4}\left|\partial_{t}^{2} \partial_{x}\left(\frac{\sigma}{\rho_{0}}\right)\right|^{2} d x \leq C\left(\mathscr{E}^{5}+\mathscr{E}^{1}\right)+C \theta_{1}^{2}\left(\mathscr{E}_{-1}^{5, \sigma}+\mathscr{E}_{a}^{3, \sigma}+\mathscr{E}^{1}+\mathscr{E}_{0}^{0, \sigma}\right) .
$$

Also, by first dividing (4-94) by $r$ and then squaring, we obtain

$$
\begin{aligned}
\mathscr{E}_{a}^{6, \sigma} & =\int\left(\delta+\frac{4 \varepsilon}{3}\right)^{2} 16 \pi^{2} r^{2}\left|\partial_{t}^{2} \partial_{x}\left(\frac{\sigma}{\rho_{0}}\right)\right|^{2} d x \\
& \leq C\left(\mathscr{E}^{6}+\mathscr{E}_{a}^{3, \sigma}+\mathscr{E}_{-1}^{2, \sigma}+\mathscr{E}^{1}\right)+C \theta_{1}^{2}\left(\mathscr{E}_{b}^{1, \sigma}+\mathscr{E}_{a}^{3, \sigma}+\mathscr{E}^{1}+\mathscr{E}_{-1}^{0, \sigma}\right) .
\end{aligned}
$$

Now we record an estimate of $\mathscr{E}^{6}$.

Lemma 4.7. There exists an $\mathscr{F}^{6}$ such that

$$
\frac{d}{d t}\left[\mathscr{E}^{6}+\widetilde{F}\right]+\mathscr{D}^{6} \leq\left(\eta+C \theta_{1}\right) \mathscr{E}^{6}+\left(\frac{1}{2}+C \theta_{1}\right) \mathscr{D}^{6}+C\left(\mathscr{E}^{5}+\mathscr{E}_{-1}^{5, \sigma}+\mathscr{E}_{b}^{1, \sigma}+\mathscr{E}_{0}^{2, \sigma}+\mathscr{E}_{0}^{0, \sigma}+\mathscr{E}^{3}+\mathscr{E}^{2}+\mathscr{E}^{1}\right) .
$$

Moreover, $\left|\mathscr{F}^{6}\right| \leq C \theta_{1}\left(\mathscr{E}^{6}+\mathscr{E}^{3}+\mathscr{E}^{1}\right)$.

Proof. The energy inequality (4-97) can be obtained by multiplying (4-90) by $\partial_{t}^{3} v$ and integrating over $x$ as done in Lemma 4.4. We omit further details.

As seen in the previous estimates in Lemmas 4.3, 4.4, 4.6, and 4.7, the time differentiation of the equation keeps the main structure of the highest-order terms as well as the boundary condition. Using the time differentiated equations (4-90) and (4-92), we can follow the line of analysis presented in these four lemmas to derive energy inequalities for $\mathscr{E}^{7}, \mathscr{C}_{a}^{7}, \mathscr{C}_{-1}^{7, \sigma}, \mathscr{E}^{8}$ and $\mathscr{E}_{a}^{8, \sigma}$. We record these in the following lemma but omit a proof.

Lemma 4.8. Let $\mathscr{E}$ be given by (4-19). We have the following estimates.

$$
\begin{aligned}
& \frac{d}{d t} \mathscr{E}^{7}+\mathscr{D}^{7} \leq\left(\eta+C \theta_{1}\right)^{\mathscr{E}}+\left(\frac{1}{2}+C \theta_{1}\right) \mathscr{D}^{7}+C \theta_{1}\left(\mathscr{E}_{a}^{6, \sigma}+\mathscr{E}^{6}+\mathscr{E}^{5}+\mathscr{E}^{3}+\mathscr{E}^{2}+\mathscr{E}^{1}\right)+C \mathscr{E}^{5}, \\
& \mathscr{E}_{a}^{7} \leq C\left(\mathscr{E}^{7}+\mathscr{E}_{a}^{6, \sigma}+\mathscr{E}^{2}\right)+C \theta_{1}\left(\mathscr{E}_{a}^{6, \sigma}+\mathscr{E}_{a}^{3, \sigma}+\mathscr{E}^{1}+\mathscr{E}^{2}+\mathscr{E}_{a_{1}^{3}, v}+\mathscr{E}_{a}^{5}\right), \\
& \frac{d}{d t} \mathscr{\mathscr { E }}_{-1}^{7, \sigma} \leq\left(\eta+C \theta_{1}\right) \mathscr{E}_{-1}^{7, \sigma}+C \mathscr{D}^{7}+C \theta_{1}^{2}\left(\mathscr{E}^{6}+\mathscr{E}^{3}+\mathscr{E}_{-1}^{5, \sigma}\right), \\
& \frac{d}{d t}\left[\mathscr{E}^{8}+\mathscr{F}^{8}\right]+\mathscr{D}^{8} \leq\left(\eta+C \theta_{1}\right)^{\mathscr{E}^{8}}+\left(\frac{1}{2}+C \theta_{1}\right) \mathscr{D}^{8}+C\left(\mathscr{E}_{-}^{8} \mathscr{E}^{8}-\mathscr{E}_{a}^{8, \sigma}\right)+C \mid \mathscr{E}^{2} \\
& \quad \text { where }\left|\mathscr{F}^{8}\right| \leq C \theta_{1} \mathscr{E}+C \mid \mathscr{E}^{2}, \\
& \mathscr{E}_{a}^{8, \sigma} \leq C\left(\mathscr{E}^{8}+\mathscr{E}_{a}^{6, \sigma}+\mathscr{E}_{-1}^{5, \sigma}+\mathscr{E}^{3}\right)+C \theta_{1}^{2}\left(\mathscr{E}_{b}^{1, \sigma}+\mathscr{E}_{a}^{3, \sigma}+\mathscr{E}^{1}+\mathscr{E}_{-1}^{0, \sigma}\right) .
\end{aligned}
$$

The next lemma ensures that the assumption (4-20) is valid within our energy $\mathscr{E}$.

Lemma 4.9. There exists a constant $\kappa>0$ such that if $\mathscr{E} \leq \kappa$, then

$$
\begin{aligned}
\left\|\frac{\sigma}{\rho_{0}}\right\|_{L^{\infty}}+\left\|\partial_{t}\left(\frac{\sigma}{\rho_{0}}\right)\right\|_{L^{\infty}}+\left\|\partial_{t}^{2}\left(\frac{\sigma}{\rho_{0}}\right)\right\|_{L^{\infty}}+\left\|\partial_{t}^{3}\left(\frac{\sigma}{\rho_{0}}\right)\right\|_{L^{\infty}} \\
+\left\|1-\frac{r_{0}}{r}\right\|_{L^{\infty}}+\left\|\rho r^{3} \partial_{x}\left(\frac{\sigma}{\rho_{0}}\right)\right\|_{L^{\infty}}+\left\|\frac{v}{r}\right\|_{L^{\infty}}+\left\|\frac{\partial_{t} v}{r}\right\|_{L^{\infty}}+\left\|\frac{\partial_{t}^{2} v}{r}\right\|_{L^{\infty}} \leq C \sqrt{\mathscr{E}},
\end{aligned}
$$


for some constant $C>0$. Here $\mathscr{E}$ is given by (4-19).

Proof. The proof proceeds in four steps.

Step $1\left(\partial_{t}^{k}\left(\sigma / \rho_{0}\right)\right.$ estimates). We begin by estimating $\sigma / \rho_{0}$ in $W^{1,1}((0, M))$. First, we use Hölder's inequality to estimate

$$
\int\left|\frac{\sigma}{\rho_{0}}\right| d x \leq \sqrt{M}\left(\int\left|\frac{\sigma}{\rho_{0}}\right| d x\right)^{1 / 2} \leq C \sqrt{\mathscr{\mathscr { E }}_{-1}^{0, \sigma}} \leq C \sqrt{\mathscr{E}} .
$$

On the other hand, we may estimate

$$
\int\left|\partial_{x}\left(\frac{\sigma}{\rho_{0}}\right)\right| d x \leq\left(\int \frac{r^{2}}{\rho}\left|\partial_{x}\left(\frac{\sigma}{\rho_{0}}\right)\right|^{2} d x\right)^{1 / 2}\left(\int \frac{\rho}{r^{2}} d x\right)^{1 / 2} \leq C\left(\mathscr{E}_{b}^{1, \sigma}\right)^{1 / 2} \leq C \sqrt{\mathscr{E}} .
$$

Here we have used the fact that $r^{2}(x) \sim x^{2 / 3}$ for $x \sim 0$, which follows from the definition of $r(x)$ and L'Hospital's rule, to see that $\int\left(\rho / r^{2}\right) d x<\infty$. Combining these estimates with the usual one-dimensional Sobolev embedding $W^{1,1}((0, M)) \hookrightarrow C^{0}((0, M))$, we find that $\frac{\sigma}{\rho_{0}} \in C^{0}$ and

$$
\left\|\frac{\sigma}{\rho_{0}}\right\|_{L^{\infty}} \leq C \sqrt{\mathscr{E}} .
$$

Now to control $\frac{\partial_{t} \sigma}{\rho_{0}}$, we argue similarly to estimate

$$
\int\left|\frac{\partial_{t} \sigma}{\rho_{0}}\right|+\left|\partial_{x}\left(\frac{\partial_{t} \sigma}{\rho_{0}}\right)\right| d x \leq C \sqrt{\mathscr{E}_{-1}^{2, \sigma}}+\sqrt{\mathscr{E}_{a}^{3, \sigma}} \leq C \sqrt{\mathscr{E}}
$$

Then $\frac{\partial_{t} \sigma}{\rho_{0}} \in C^{0}$ and

$$
\left\|\frac{\partial_{t} \sigma}{\rho_{0}}\right\|_{L^{\infty}} \leq C \sqrt{\mathscr{E}}
$$

A similar argument, employing $\mathscr{E}_{-1}^{1+2 i, \sigma}$ and $\mathscr{E}_{a}^{2+2 i, \sigma}$ for $i=1,2$, then implies that

$$
\frac{\partial_{t}^{2} \sigma}{\rho_{0}}, \frac{\partial_{t}^{3} \sigma}{\rho_{0}} \in C^{0} \quad \text { and } \quad\left\|\frac{\partial_{t}^{2} \sigma}{\rho_{0}}\right\|_{L^{\infty}}+\left\|\frac{\partial_{t}^{3} \sigma}{\rho_{0}}\right\|_{L^{\infty}} \leq C \sqrt{\mathscr{E}}
$$

We thus deduce from (4-106) and (4-108)-(4-109) that

$$
\left\|\frac{\sigma}{\rho_{0}}\right\|_{L^{\infty}}+\left\|\partial_{t}\left(\frac{\sigma}{\rho_{0}}\right)\right\|_{L^{\infty}}+\left\|\partial_{t}^{2}\left(\frac{\sigma}{\rho_{0}}\right)\right\|_{L^{\infty}}+\left\|\partial_{t}^{3}\left(\frac{\sigma}{\rho_{0}}\right)\right\|_{L^{\infty}} \leq C \sqrt{\mathscr{E}} .
$$

Step $2\left(1-r_{0} / r\right.$ estimate). Let us now suppose that $\mathscr{E} \leq \kappa$ with $\kappa$ small enough that $C \sqrt{\mathscr{E}} \leq \frac{1}{2}$, where $C>0$ is the constant appearing on the right side of (4-110). In particular, this implies that $\left\|\sigma / \rho_{0}\right\|_{L^{\infty}} \leq \frac{1}{2}<1$. With this estimate in hand, we can derive an estimate for $r_{0} / r$. Indeed, the Taylor expansion (4-21) easily implies the estimate

$$
\left\|1-\frac{r_{0}}{r}\right\|_{L^{\infty}} \leq C\left\|\frac{\sigma}{\rho_{0}}\right\|_{L^{\infty}}^{1+k} \leq C \sqrt{\mathscr{E}}
$$

for some $k \geq 0$. This is the $1-r_{0} / r$ estimate in (4-103). 
Step 3 ( $\partial_{t}^{k} v / r$ estimates). We now turn to estimates for $\partial_{t}^{k} v / r, k=0,1,2$. From Step 1 , we know that $\sigma / \rho_{0}$ and $\partial_{t}\left(\sigma / \rho_{0}\right)$ are continuous and bounded, while from Step 2, we know that $\left\|\sigma / \rho_{0}\right\|_{L^{\infty}} \leq \frac{1}{2}$, so that $1+\sigma / \rho_{0}$ is also continuous and bounded. From the boundary conditions at $x=0$, we also have that $r^{2} v(0, t)=0$. Hence we may spatially integrate the continuity equation (4-4) to see that

$$
\left(r^{2} v\right)(x, t)=\frac{-1}{4 \pi} \int_{0}^{x} \frac{1}{\rho_{0}(y)\left(1+\frac{\sigma(y, t)}{\rho_{0}(y)}\right)^{2}} \frac{\partial_{t} \sigma(y, t)}{\rho_{0}(y)} d y .
$$

Due to the asymptotics (1-24), we now have that

$$
\int_{0}^{M} \frac{d y}{\rho_{0}(y)}<\infty
$$

This and the estimates (4-110) then imply that $\frac{v}{r} \in C^{0}$ and

$$
\left\|r^{2} v\right\|_{L^{\infty}} \leq C\left\|\partial_{t} \frac{\sigma}{\rho_{0}}\right\|_{L^{\infty}} \leq C \sqrt{\mathscr{E}}
$$

On the other hand, due to L'Hospital, we have that

$$
\frac{1}{r^{3}(x, t)} \int_{0}^{x} \frac{d y}{\rho_{0}(y)} \sim \frac{4 \pi \rho(x, t)}{3 \rho_{0}(x)}=\frac{4 \pi}{3}\left(1+\frac{\sigma(x, t)}{\rho_{0}(y)}\right)<\infty \quad \text { for } x \sim 0,
$$

which means that

$$
\sup _{x \in(0, M)} \frac{1}{r^{3}(x, t)} \int_{0}^{x} \frac{d y}{\rho_{0}(y)}<\infty .
$$

We may then deduce that $\frac{v}{r} \in C^{0}$ and

$$
\left\|\frac{v}{r}\right\|_{L^{\infty}} \leq C\left\|\partial_{t} \frac{\sigma}{\rho_{0}}\right\|_{L^{\infty}} \sup _{x \in(0, M)} \frac{1}{r^{3}(x, t)} \int_{0}^{x} \frac{d y}{\rho_{0}(y)} \leq C \sqrt{\mathscr{E}} .
$$

Now we apply $\partial_{t}$ to (4-4) and argue as above to see that

$$
\begin{aligned}
\left(r^{2} \partial_{t} v\right)(x, t)=- & \int_{0}^{x} \frac{1}{4 \pi \rho_{0}(y)\left(1+\frac{\sigma(y, t)}{\rho_{0}(y)}\right)^{2}} \frac{\partial_{t}^{2} \sigma(y, t)}{\rho_{0}(y)} d y \\
& +\int_{0}^{x} \frac{1}{2 \pi \rho_{0}(y)\left(1+\frac{\sigma(y, t)}{\rho_{0}(y)}\right)^{3}}\left|\frac{\partial_{t} \sigma(y, t)}{\rho_{0}(y)}\right|^{2} d y-\int_{0}^{x} 2\left(r^{2} v\right)(y, t) \frac{v(y, t)}{r(y, t)} d y .
\end{aligned}
$$

Using this, we may argue as above (using estimates (4-114) and (4-117)) to deduce $r^{2} \partial_{t} v, \frac{\partial_{t} v}{r} \in C^{0}$ and

$$
\left\|r^{2} \partial_{t} v\right\|_{L^{\infty}}+\left\|\frac{\partial_{t} v}{r}\right\|_{L^{\infty}} \leq C \sqrt{\mathscr{E}}
$$


An iterative argument, using $\partial_{t}^{2}$ applied to (4-4) in conjunction with the estimates (4-119), then allows us to see that $r^{2} \partial_{t}^{2} v, \partial_{t}^{2} v / r \in C^{0}$ with

$$
\left\|r^{2} \partial_{t}^{2} v\right\|_{L^{\infty}}+\left\|\frac{\partial_{t}^{2} v}{r}\right\|_{L^{\infty}} \leq C \sqrt{\mathscr{E}}
$$

Then (4-114), (4-117), and (4-119)-(4-120) may be combined to derive the $\frac{\partial_{t}^{k} v}{r}$ estimates recorded in (4-103).

Step $4\left(\rho r^{3} \partial_{x}\left(\sigma / \rho_{0}\right)\right.$ estimate). Since $\left\|\sigma / \rho_{0}\right\|_{L^{\infty}} \leq \frac{1}{2}$, to prove the $\rho r^{3} \partial_{x}\left(\sigma / \rho_{0}\right)$ estimate listed in (4-103), it suffices to estimate this term with $\rho$ replaced by $\rho_{0}$. We claim that

$$
\left\|\rho_{0} r^{3} \partial_{x}\left(\frac{\sigma}{\rho_{0}}\right)\right\|_{L^{\infty}} \leq C\left(\sqrt{\mathscr{E}_{b}^{1, \sigma}}+\sqrt{\mathscr{E} 4}\right) \leq C \sqrt{\mathscr{E}}
$$

To prove (4-121), we will use the one-dimensional Sobolev embedding $W^{1,1} \hookrightarrow C^{0}$. First note that

$$
\int \rho_{0} r^{3}\left|\partial_{x}\left(\frac{\sigma}{\rho_{0}}\right)\right| d x \leq\left(\int \rho \rho_{0}^{2} r^{2} d x\right)^{1 / 2}\left(\int \frac{r^{2}}{\rho}\left|\partial_{x}\left(\frac{\sigma}{\rho_{0}}\right)\right|^{2} d x\right)^{1 / 2} \leq C \sqrt{\mathscr{E}_{b}^{1, \sigma}} .
$$

On the other hand, we may compute

$$
\begin{aligned}
\partial_{x}\left(\rho_{0} r^{3} \partial_{x}\left(\frac{\sigma}{\rho_{0}}\right)\right) & =\frac{\rho_{0}}{r} \partial_{x}\left(r^{4} \partial_{x}\left(\frac{\sigma}{\rho_{0}}\right)\right)+\partial_{x} \rho_{0} r^{3} \partial_{x}\left(\frac{\sigma}{\rho_{0}}\right)-\frac{\rho_{0}}{4 \pi \rho} \partial_{x}\left(\frac{\sigma}{\rho_{0}}\right), \\
\partial_{x} \rho_{0} & =-\frac{x}{4 \pi K \gamma \rho_{0}^{\gamma-1} r_{0}^{4}} .
\end{aligned}
$$

Then since $\frac{\rho_{0}}{\rho_{0}^{2 \gamma-2}} \leq \frac{C}{\rho_{0}}$ as long as $\gamma<2$, we may estimate

$$
\begin{aligned}
\int \rho_{0} r_{0}^{2}\left|\partial_{x}\left(\rho_{0} r^{3} \partial_{x}\left(\frac{\sigma}{\rho_{0}}\right)\right)\right|^{2} d x \\
\quad \leq C \int \rho_{0} r_{0}^{2}\left[\frac{\rho_{0}^{2}}{r^{2}}\left|\partial_{x}\left(r^{4} \partial_{x}\left(\frac{\sigma}{\rho_{0}}\right)\right)\right|^{2}+\frac{\rho_{0}^{2}}{\rho^{2}}\left|\partial_{x}\left(\frac{\sigma}{\rho_{0}}\right)\right|^{2}+\frac{x^{2} r^{6}}{\rho_{0}^{2 \gamma-2} r_{0}^{8}}\left|\partial_{x}\left(\frac{\sigma}{\rho_{0}}\right)\right|^{2}\right] d x \\
\leq C^{\mathscr{E}} \mathbb{E}^{4}+\mathscr{E}_{b}^{1, \sigma} .
\end{aligned}
$$

Then from this and Hölder's inequality, we get

$$
\begin{aligned}
\int\left|\partial_{x}\left(\rho_{0} r^{3} \partial_{x}\left(\frac{\sigma}{\rho_{0}}\right)\right)\right| d x & \leq\left(\int \frac{d x}{\rho_{0} r_{0}^{2}}\right)^{1 / 2}\left(\int \rho_{0} r_{0}^{2}\left|\partial_{x}\left(\rho_{0} r^{3} \partial_{x}\left(\frac{\sigma}{\rho_{0}}\right)\right)\right|^{2} d x\right)^{1 / 2} \\
& \leq C \sqrt{\mathscr{E}^{4}}+\sqrt{\mathscr{E}_{b}^{1, \sigma}}
\end{aligned}
$$

Together, the estimates (4-123) and (4-125) constitute a $W^{1,1}$ estimate for $\rho_{0} r^{3} \partial_{x}\left(\frac{\sigma}{\rho_{0}}\right)$, so we then obtain (4-121) via the Sobolev embedding. 


\section{Nonlinear instability}

5A. The bootstrap argument. Based on the nonlinear estimates in the previous section, we now establish a bootstrap argument that allows us to control the growth of $\mathscr{E}$ in terms of the linear growth rate $\lambda$, constructed in Theorem 2.1. The idea is to assume small data and that the lowest-order energy, $\mathscr{C}^{\circ}$, grows no faster than the linear growth rate; then the inequalities in the last section allow for a bootstrap argument that shows that all of $\mathscr{E}$ grows no faster than the linear growth rate.

Proposition 5.1. Let $\sigma$ and $v$ be a solution to the Navier-Stokes-Poisson system (4-2). Assume that

$$
\sqrt{\mathscr{E}}(0) \leq C_{0} \iota \quad \text { and } \quad \sqrt{\mathscr{E} 0}(t) \leq C_{0} \iota e^{\lambda t} \quad \text { for } 0 \leq t \leq T,
$$

where $\mathscr{E}^{0}$ and $\mathscr{E}$ are as defined in (4-11) and (4-19). Then there exist $C_{\star}$ and $\theta_{\star}>0$ such that if $0 \leq t \leq \min \left\{T, T\left(\iota, \theta_{\star}\right)\right\}$, then

$$
\sqrt{\mathscr{E}}(t) \leq C_{\star} l e^{\lambda t} \leq C_{\star} \theta_{\star},
$$

where we have written $T\left(\iota, \theta_{\star}\right)=\frac{1}{\lambda} \ln \frac{\theta_{\star}}{\iota}$.

Proof. To prove the result, we will employ a bootstrap argument using all of the nonlinear energy estimates derived in the previous section. We now choose $\theta_{1}$ and $\eta$ sufficiently small in all of these estimates that $C \theta_{1}+\eta \leq \lambda / 2$ and $C \theta_{1} \leq \frac{1}{8}$ in all of the energy inequalities. Throughout this proof, we will write $\tilde{C}$ for a generic constant; we write this in place of $C$ to distinguish the constants from those appearing in the nonlinear energy estimates.

To begin the bootstrapping, we show that the estimate (5-1) allows us to control an integral of the $\mathscr{D}^{0}$ dissipation. Indeed, we use (4-25) and (5-1) along with Gronwall's inequality to see that for $0 \leq t \leq T$,

$$
\frac{d}{d t} \mathscr{E}^{0}+\frac{1}{2} \mathscr{D}^{0} \leq C_{\mathscr{E}}^{\mathscr{C}} \leq C C_{0}^{2}\left(\iota e^{\lambda t}\right)^{2}+\frac{\lambda}{2} \mathscr{E}^{0} \Rightarrow \frac{1}{2} \int_{0}^{t} e^{\lambda / 2(t-s)} \mathscr{D}^{0}(s) d s \leq \tilde{C} \iota^{2} e^{2 \lambda t} .
$$

Then we employ (4-34) with $\beta=-1$ in conjunction with (5-3) to see that

$$
\begin{aligned}
\frac{d}{d t}\left(e^{-t \lambda / 2 \mathscr{C}_{-1} 0, \sigma}(t)\right) \leq C e^{-t \lambda / 2} \mathscr{D}^{0}(t) & \Rightarrow \mathscr{\mathscr { C }}_{-1}^{0, \sigma}(t) \leq \mathscr{E}_{-1}^{0, \sigma}(0) e^{t \lambda / 2}+C \int_{0}^{t} e^{\lambda / 2(t-s)} \mathscr{D}^{0}(s) d s \\
& \Rightarrow \mathscr{E}_{-1}^{\mathscr{\mathscr { C }} 0, \sigma}(t) \leq \tilde{C} \iota^{2} e^{2 \lambda t} \Longrightarrow \mathscr{E}_{0}^{0, \sigma}(t) \leq \tilde{C}_{\mathscr{C}}^{\mathscr{C} 0, \sigma}(t) \leq \tilde{C} \iota^{2} e^{2 \lambda t} .
\end{aligned}
$$

Next, let $q>0$ be the constant from estimate (4-35) and choose $k=(4 q) / \lambda$. Then (4-35) and (4-47), together with (4-25) and the above estimates, imply that for $0 \leq t \leq T$,

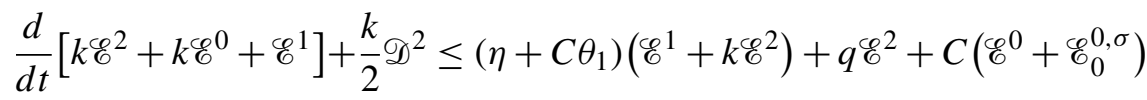

$$
\begin{aligned}
& \leq \frac{\lambda}{2}\left(\mathscr{E}^{1}+k^{\mathscr{E}} \mathscr{E}^{2}+k^{\mathscr{E}} 0\right)+\tilde{C} \iota^{2} e^{2 \lambda t} .
\end{aligned}
$$

Using Gronwall's inequality again, we obtain from this that for $0 \leq t \leq T$,

$$
\mathscr{E}^{2}(t)+\mathscr{E}^{1}(t)+\frac{1}{2} \int_{0}^{t} e^{\lambda / 2(t-s)} \mathscr{D}^{2}(s) d s \leq \tilde{C} l^{2} e^{2 \lambda t} .
$$


Here we have used the fact that $k$ is bounded and nonzero to absorb it into the constant $\tilde{C}$. We then employ (4-56) with $\beta=-1$ to see that

$$
\begin{aligned}
& \frac{d}{d t}\left(e^{-t \lambda / 2_{\mathscr{E}}^{2, \sigma}}(t)\right) \leq C e^{-t \lambda / 2}\left(\mathscr{D}^{2}(t)+\mathscr{D}^{0}(t)\right) \\
& \Rightarrow \mathscr{E}_{-1}^{2, \sigma}(t) \leq \mathscr{E}_{-1}^{2, \sigma}(0) e^{t \lambda / 2}+C \int_{0}^{t} e^{\lambda / 2(t-s)}\left(\mathscr{D}^{2}(s)+\mathscr{D}^{0}(s)\right) d s \\
& \Rightarrow \mathscr{E}_{-1}^{2, \sigma}(t) \leq \tilde{C} \iota^{2} e^{2 \lambda t} \Rightarrow \mathscr{E}_{0}^{2, \sigma}(t) \leq \tilde{C}_{\mathscr{E}}^{\mathscr{E}}{ }_{-1}^{2, \sigma}(t) \leq \tilde{C} \iota^{2} e^{2 \lambda t}
\end{aligned}
$$

Bootstrapping further, (4-57) gives rise to

$$
\mathscr{E}^{3}(t) \leq \tilde{C} \iota^{2} e^{2 \lambda t}
$$

Similarly, from (4-72), (4-73), (4-74), (4-76), and (4-77), we also obtain, for $0 \leq t \leq T$,

$$
\mathscr{E}_{b}^{0, r}(t)+\mathscr{E}_{b}^{1, \sigma}(t)+\mathscr{E}_{a}^{3, \sigma}(t)+\mathscr{E}_{a_{1}}^{3, v}(t)+\mathscr{E}_{a_{2}}^{3, v}(t) \leq \tilde{C} \iota^{2} e^{2 \lambda t} .
$$

Next, from (4-78), we get

$$
\frac{d}{d t} \mathscr{E}^{4} \leq\left(\eta+C \theta_{1}\right)^{\mathscr{E}} \mathscr{E}^{4}+C \iota^{2} e^{2 \lambda t} \Longrightarrow \mathscr{E}^{4}(t) \leq \tilde{C} \iota^{2} e^{2 \lambda t} .
$$

In turn, from (4-86) and (4-88), we find that

$$
\mathscr{E}_{a_{1}}^{4}(t)+\mathscr{E}_{a_{2}}^{4}(t) \leq \tilde{C} \iota^{2} e^{2 \lambda t}
$$

Similarly, the energy inequalities (4-89), (4-91), (4-93) yield

$$
\mathscr{E}^{5}(t)+\mathscr{E}_{a}^{5}(t)+\mathscr{E}_{-1}^{5, \sigma}(t) \leq \tilde{C} \iota^{2} e^{2 \lambda t},
$$

and (4-97) and (4-96) yield

$$
\mathscr{E}^{6}(t)+\mathscr{E}_{a}^{6, \sigma}(t) \leq \tilde{C} \iota^{2} e^{2 \lambda t}
$$

Successively, (4-98), (4-99), and (4-100) imply

$$
\mathscr{E}^{7}(t)+\mathscr{E}_{a}^{7}(t)+\mathscr{E}_{-1}^{7, \sigma}(t) \leq \tilde{C} \iota^{2} e^{2 \lambda t} .
$$

To get the bound of $\mathscr{E}^{8}$, we first note that $\mathscr{E}^{8}$ satisfies the following inequality from (4-101) and (4-102):

$$
\frac{d}{d t}\left[\mathscr{E}^{8}+\widetilde{F}_{*}\right] \leq\left(\eta+C \theta_{1}\right)\left(\mathscr{E}^{8}+\widetilde{F}_{*}\right)+C_{1}|\mathscr{E}|^{2}+\widetilde{C} \iota^{2} e^{2 \lambda t}
$$

for some constants $C_{1}>0$ and $\widetilde{C}>0$. We now define $T^{*}$ by

$$
T^{*}:=\sup \left\{t \mid \mathscr{E}(s) \leq \min \left\{\theta_{1}, \frac{\lambda}{4 C_{1}}\right\} \text { for } s \in[0, t]\right\} .
$$

Let $0 \leq t \leq \min \left\{T, T^{*}\right\}$. Then by the Gronwall inequality, (5-15) implies that

$$
\mathscr{E}^{8}(t)+\widetilde{F}_{*}(t) \leq \tilde{C} \iota^{2} e^{2 \lambda t} \Longrightarrow \mathscr{E}^{8}(t)+\mathscr{E}_{a}^{8, \sigma}(t) \leq \tilde{C} \iota^{2} e^{2 \lambda t} \quad \text { for } 0 \leq t \leq \min \left\{T, T^{*}\right\} .
$$


Thus, combining all of the above analysis, we finally obtain

$$
\mathscr{E}(t) \leq C_{2} \iota^{2} e^{2 \lambda t} \quad \text { for } 0 \leq t \leq \min \left\{T, T^{*}\right\}
$$

for a constant $C_{2}>0$ independent of $\iota$.

We now choose $\theta_{\star}$ such that $C_{2}\left(\theta_{\star}\right)^{2}<\min \left\{\theta_{1}, \lambda / 4 C_{1}\right\}$. We consider the following two cases.

(i) $T\left(\iota, \theta_{\star}\right) \leq \min \left\{T, T^{*}\right\}$. In this case, the conclusion follows without any additional work.

(ii) $T\left(\iota, \theta_{\star}\right)>\min \left\{T, T^{*}\right\}$. We claim that it must hold that $T \leq T^{*}<T\left(\iota, \theta_{*}\right)$, in which case the conclusion directly follows. To prove the claim, we note that otherwise we would have $T^{*}<T<T\left(\iota, \theta_{\star}\right)$. Letting $t=T^{*}$, from (5-18), we get

$$
\mathscr{E}\left(T^{*}\right) \leq C_{2} \iota^{2} e^{2 \lambda T^{*}}<C_{2} \iota^{2} e^{2 \lambda T^{\iota}}=C_{2}\left(\theta_{\star}\right)^{2} \text { by the definition of } T\left(\iota, \theta_{\star}\right),
$$

but this is impossible due to our choice of $\theta_{\star}$ since it would then contradict the definition of $T^{*}$. Since we then find our desired estimate in both cases, this concludes the proof of the proposition.

5B. Further nonlinear estimates. As preparation for the proof of our main theorem, we recall that the Navier-Stokes-Poisson system (4-2) can be written in perturbed form as in (3-38) and (3-39) in terms of $\sigma$ and $w:=r^{2} v$ :

$$
\partial_{t}\left(\begin{array}{c}
\sigma \\
w
\end{array}\right)-\mathscr{L}\left(\begin{array}{c}
\sigma \\
w
\end{array}\right)=\left(\begin{array}{c}
N_{1} \\
N_{2}
\end{array}\right)
$$

with the boundary conditions

$$
\left(\frac{w}{r^{2}}\right)(0, t)=0 \quad \text { and } \quad \sigma(M, t)=0, \quad \mathscr{B}(w)=N_{\mathscr{B}} \quad \text { at } \quad x=M,
$$

where the boundary operator $\mathscr{B}(w)$ is defined by (3-34), $N_{\mathscr{B}}$ is given as

$$
N_{\mathscr{B}}=\left.\left\{\left(\delta+\frac{4 \varepsilon}{3}\right) 4 \pi \sigma \partial_{x} w-4 \varepsilon\left[\left(\frac{r_{0}}{r}\right)^{3}-1\right] \frac{w}{r_{0}^{3}}\right\}\right|_{x=M},
$$

and $N_{1}$ and $N_{2}$ become

$$
\begin{aligned}
& N_{1}=-4 \pi\left(2 \rho_{0}+\sigma\right) \sigma \partial_{x} w, \\
& N_{2}=\frac{2 w^{2}}{r^{3}}-4 \pi\left(r^{4}-r_{0}^{4}\right) \partial_{x}\left(K \gamma \rho_{0}^{\gamma} \frac{\sigma}{\rho_{0}}\right)-4 \pi r^{4} \partial_{x}\left(\rho_{0}^{\gamma} a_{*}\left(\frac{\sigma}{\rho_{0}}\right)^{2}\right)-M_{1}-M_{2},
\end{aligned}
$$

where

$$
\begin{aligned}
M_{1}= & \frac{x}{r_{0}^{4}}\left(r_{0}^{4}-r^{4}-\frac{r_{0}}{\pi} \int_{0}^{x} \frac{\sigma}{\rho_{0}^{2}} d y\right) \\
= & x\left\{\frac{c_{1}}{r_{0}^{3}} \int_{0}^{x} \frac{1}{\rho_{*}}\left(\frac{\sigma}{\rho_{0}}\right)^{2} d y+\frac{c_{2}}{r_{0}^{6}}\left(\int_{0}^{x} \frac{\sigma}{\rho_{0}^{2}} d y\right)^{2}\right\} \text { by Taylor expansion, } \\
& \text { where } \rho_{*} / \rho_{0} \sim 1 \text { is a bounded smooth function of } \frac{\sigma}{\rho_{0}}
\end{aligned}
$$


and

$$
\begin{aligned}
M_{2} & =16 \pi^{2}\left(\delta+\frac{4 \varepsilon}{3}\right)\left\{r_{0}^{4} \partial_{x}\left(\rho_{0} \partial_{x} w\right)-r^{4} \partial_{x}\left(\rho \partial_{x} w\right)\right\} \\
& =16 \pi^{2}\left(\delta+\frac{4 \varepsilon}{3}\right)\left\{\left(r_{0}^{4}-r^{4}\right) \partial_{x}\left(\rho_{0} \partial_{x} w\right)-r^{4} \partial_{x}\left(\sigma \partial_{x} w\right)\right\} .
\end{aligned}
$$

It is possible to estimate these nonlinearities in terms of the energy $\mathscr{E}$ given by (4-19). We present these estimates now.

Lemma 5.2. For each $t$,

$$
\mathfrak{E}\left(N_{1}, N_{2}\right) \leq C|\mathscr{E}|^{2} \quad \text { and } \quad\left|N_{\mathscr{B}}\right|+\left|\partial_{t} N_{\mathscr{B}}\right|+\left|\partial_{t}^{2} N_{\mathscr{B}}\right| \leq C|\mathscr{E}|^{2},
$$

where $\mathfrak{E}$ is defined in (3-46).

Proof. The second inequality follows directly from Lemma 4.9. For the first inequality, we only provide the details for the highest-order nonlinear term $M_{2}$ in $N_{2}$. Lower-order terms may be estimated similarly. Throughout the proof, we will write $\theta_{1}$ to denote the left side of estimate (4-103); Lemma 4.9 then implies that $\theta_{1} \leq C \sqrt{\mathscr{E}}$.

By rewriting $M_{2}$ as

$$
\begin{aligned}
& \frac{M_{2}}{16 \pi^{2}\left(\delta+\frac{4 \varepsilon}{3}\right)} \\
& =\left(r_{0}^{4}-r^{4}\right) \partial_{x}\left(\rho_{0} \partial_{x} w\right)-r^{4} \partial_{x}\left(\sigma \partial_{x} w\right) \\
& =\left[\left(\frac{r_{0}}{r}\right)^{4}-1\right]\left\{\frac{\rho_{0}}{\rho} r^{4} \partial_{x}\left(\rho \partial_{x} w\right)+r^{4} \partial_{x}\left(\frac{\rho_{0}}{\rho}\right) \rho \partial_{x} w\right\}-\frac{\sigma}{\rho} r^{4} \partial_{x}\left(\rho \partial_{x} w\right)-r^{4} \partial_{x}\left(\frac{\sigma}{\rho}\right) \rho \partial_{x} w,
\end{aligned}
$$

it is easy to see that

$$
\int \frac{\left|N_{2}\right|^{2}}{r_{0}^{4}} d x \leq C \theta_{1}^{2 \mathscr{E}} \leq C|\mathscr{E}|^{2}
$$

Next,

$$
\begin{gathered}
\frac{\partial_{x} M_{2}}{16 \pi^{2}\left(\delta+\frac{4 \varepsilon}{3}\right)}=\left(\frac{r_{0}}{r}\right)^{3} \frac{1-\left(\frac{r_{0}}{r}\right)^{3}+\frac{\sigma}{\rho_{0}}}{\pi \rho r_{0}^{2} r}\left\{\frac{\rho_{0}}{\rho} r^{4} \partial_{x}\left(\rho \partial_{x} w\right)+r^{4} \partial_{x}\left(\frac{\rho_{0}}{\rho}\right) \rho \partial_{x} w\right\} \\
+\left[\left(\frac{r_{0}}{r}\right)^{4}-1\right]\left\{\frac{\rho_{0}}{\rho} \partial_{x}\left(r^{4} \partial_{x}\left(\rho \partial_{x} w\right)\right)+2 r^{4} \partial_{x}\left(\frac{\rho_{0}}{\rho}\right) \partial_{x}\left(\rho \partial_{x} w\right)+\partial_{x}\left(r^{4} \partial_{x}\left(\frac{\rho_{0}}{\rho}\right)\right) \rho \partial_{x} w\right\} \\
-\frac{\sigma}{\rho} \partial_{x}\left(r^{4} \partial_{x}\left(\rho \partial_{x} w\right)\right)-2 r^{4} \partial_{x}\left(\frac{\sigma}{\rho}\right) \partial_{x}\left(\rho \partial_{x} w\right)-\partial_{x}\left(r^{4} \partial_{x}\left(\frac{\sigma}{\rho}\right)\right) \rho \partial_{x} w .
\end{gathered}
$$

Hence, from the definition of the energies and from the estimates in the previous section,

$$
\int \rho_{0}\left|\partial_{x} M_{2}\right|^{2} d x \leq C \theta_{1}^{2}\left(\mathscr{E}_{a_{2}}^{4}+\mathscr{E}_{a_{1}}^{3, v}+\mathscr{E}_{b}^{1, \sigma}\right)+C \theta_{1}^{4}\left(\mathscr{E}^{4}+\mathscr{E}_{b}^{1, \sigma}\right)+C\left(\mathscr{E}_{b}^{1, \sigma}+\mathscr{E}^{4}\right) \mathscr{E}_{a_{1}}^{3, v} \leq C\left|\mathscr{E}^{2}\right|^{2}
$$


On the other hand, $\partial_{t} M_{2}$ reads as

$$
\begin{gathered}
\frac{\partial_{t} M_{2}}{16 \pi^{2}\left(\delta+\frac{4 \varepsilon}{3}\right)}=-4 r^{3} v \partial_{x}\left(\rho \partial_{x} w\right)-r^{4} \partial_{x}\left(\partial_{t} \sigma \partial_{x} w\right)-r^{4} \partial_{x}\left(\sigma \partial_{x} \partial_{t} w\right)+\left(r_{0}^{4}-r^{4}\right) \partial_{x}\left(\rho_{0} \partial_{x} \partial_{t} w\right) \\
=-4 \frac{v}{r} r^{4} \partial_{x}\left(\rho \partial_{x} w\right)-\frac{\partial_{t} \sigma}{\rho} r^{4} \partial_{x}\left(\rho \partial_{x} w\right)-r^{4} \partial_{x}\left(\frac{\partial_{t} \sigma}{\rho}\right) \rho \partial_{x} w-r^{4} \frac{\sigma}{\rho} \partial_{x}\left(\rho \partial_{x} \partial_{t} w\right) \\
-r^{4} \partial_{x}\left(\frac{\sigma}{\rho}\right) \rho \partial_{x} \partial_{t} w+\left(r_{0}^{4}-r^{4}\right) \frac{\rho_{0}}{\rho} \partial_{x}\left(\rho \partial_{x} \partial_{t} w\right)+\left(r_{0}^{4}-r^{4}\right) \partial_{x}\left(\frac{\rho_{0}}{\rho}\right) \rho \partial_{x} \partial_{t} w
\end{gathered}
$$

Thus

$$
\int \frac{\left|\partial_{t} M_{2}\right|^{2}}{r_{0}^{4}} d x \leq C \theta_{1}^{2}\left(\mathscr{E}_{a_{1}}^{3, v}+\mathscr{E}_{a}^{3, \sigma}+\mathscr{E}_{a}^{5}\right)+C\left(\mathscr{E}^{4}+\mathscr{E}_{b}^{1, \sigma}\right) \mathscr{E}_{a}^{6, \sigma} \leq C\left|\mathscr{E}^{6}\right|^{2}
$$

5C. Data analysis. In order to prove our nonlinear instability result, we want to use the linear growing mode solutions constructed in Theorem 2.1 to construct small initial data for the nonlinear problem, written in the perturbation formulation (5-20). Small data in the perturbation formulation correspond to initial data for (1-17)-(1-20) that are close to the stationary solutions $\rho=\rho_{0}, v=0, r=r_{0}$. Unfortunately, due to the regularity framework (given by $\mathscr{E}$ as in (4-19)) in which we have proved our nonlinear estimates, we cannot simply set the initial data for the nonlinear problem (5-20) to be a small constant times the linear growing modes. The reason for this is that the initial data for the nonlinear problem must satisfy certain nonlinear compatibility conditions in order for us to guarantee local existence in the energy space defined by $\mathscr{E}$. Until now, we have taken the local well-posedness theory for the nonlinear problem for granted, but we must now say a few words about the compatibility conditions in order to construct our desired initial data.

Recall that we can rewrite the nonlinear problem (1-17)-(1-20) in the form (5-20)-(5-21) with nonlinearities given by (5-22)-(5-23). Let us concisely rewrite (5-20) as

$$
\partial_{t} \mathfrak{X}+\mathscr{L} \mathfrak{X}=\mathfrak{N}(\mathfrak{X}) \quad \text { for } \mathfrak{X}=\left(\begin{array}{c}
\sigma \\
\bar{w}
\end{array}\right) \text {, }
$$

where $\mathfrak{N}(\mathfrak{X})$ is the nonlinearity given in terms of $N_{1}$ and $N_{2}$ by the right side of (5-20). We will also rewrite the boundary conditions $(5-21)$ as

$$
\mathfrak{C}(\mathfrak{X}):=\left(\begin{array}{c}
\left.\left(w / r_{0}^{2}\right)\right|_{x=0} \\
\left.\sigma\right|_{x=M} \\
\left.\mathscr{B}(w)\right|_{x=M}
\end{array}\right)=\left(\begin{array}{c}
\left.w\left(r_{0}^{-2}-r^{-2}\right)\right|_{x=0} \\
0 \\
N_{\mathscr{B}}
\end{array}\right):=\mathfrak{N}_{\mathscr{B}}(\mathfrak{X}) .
$$

Here $r$ is determined as a nonlinear function of $\sigma$ as usual.

Rewriting the nonlinear problem as (5-32)-(5-33) now allows us to easily describe the compatibility conditions for the initial data. Given $\mathfrak{X}(0)$ as initial data for $\mathfrak{X}$ at $t=0$, we can use (5-32) to iteratively 
solve for $\partial_{t}^{j} \mathfrak{X}(0)$ for $j \geq 1$ :

$$
\begin{aligned}
\partial_{t} \mathfrak{X}(0) & =-\mathscr{L} \mathfrak{X}(0)+\mathfrak{N}(\mathfrak{X}(0)), \\
\partial_{t}^{2} \mathfrak{X}(0) & =-\mathscr{L} \partial_{t} \mathfrak{X}(0)+D \mathfrak{N}(\mathfrak{X}(0)) \cdot \partial_{t} \mathfrak{X}(0) \\
& =-\mathscr{L}(-\mathscr{L} \mathfrak{X}(0)+\mathfrak{N}(\mathfrak{X}(0)))+D \mathfrak{N}(\mathfrak{X}(0)) \cdot(-\mathscr{L} \mathfrak{X}(0)+\mathfrak{N}(\mathfrak{X}(0))),
\end{aligned}
$$

and so on for higher derivatives, where $D$ is the derivative of the nonlinearity. We may similarly compute $\partial_{t}^{j} \mathfrak{N}_{\mathscr{B}}(\mathfrak{X})(0)$ :

$$
\partial_{t} \mathfrak{N}_{\mathscr{B}}(\mathfrak{X})(0)=D \mathfrak{N}_{\mathscr{B}}(\mathfrak{X}(0)) \cdot \partial_{t} X(0)=\left.D \mathfrak{N}_{\mathscr{B}}(\mathfrak{X}(0)) \cdot[-\mathscr{L} \mathfrak{X}(0)+\mathfrak{N}(\mathfrak{X}(0))]\right|_{x=M},
$$

continuing as above for higher derivatives. This procedure may be carried out indefinitely as long as $\mathfrak{X}(0)$ is sufficiently smooth. However, we may also differentiate the boundary condition (5-33) with respect to time and then set $t=0$ to see that the data must satisfy the boundary conditions

$$
\mathfrak{C}\left(\partial_{t}^{j} \mathfrak{X}(0)\right)=\partial_{t}^{j} \mathfrak{N}_{\mathscr{B}}(\mathfrak{X})(0) \quad \text { for } j \geq 0 .
$$

Since the terms $\partial_{t}^{j} \mathfrak{X}(0)$ and $\partial_{t} \mathfrak{N}_{\mathscr{B}}(\mathfrak{X})(0)$ constructed in (5-34)-(5-35) are determined entirely by $\mathfrak{X}(0)$, we then find that the data $\mathfrak{X}(0)$ must satisfy the nonlinear compatibility conditions given by substituting (5-34)-(5-35) into (5-36).

For completely smooth solutions to the nonlinear problem, the compatibility conditions would have to hold for all $j \geq 0$. In our case, we only require solutions to remain in the energy space defined by $\mathscr{E}$, and as such, we must only solve for $\partial_{t}^{j} \mathfrak{X}(0)$ for $j=1,2,3$, given $\mathfrak{X}(0)$. This then requires the compatibility condition from (5-36) only for $0 \leq j \leq 3$. Of course, in order to guarantee that $\mathscr{E}(0)$ is finite, we must have that $\partial_{t}^{j} \mathfrak{X}(0), 0 \leq j \leq 3$, satisfies the integrability conditions in the definition of $\mathscr{E}(0)$. This in turn gives us a natural Hilbert function space $\mathbb{U}$ with the following three properties. First, if $\mathfrak{X}(0) \in \mathbb{U}$, then we have the trace estimates needed to make sense of the boundary conditions in (5-36) for $0 \leq j \leq 3$. Second, if $\|\mathfrak{X}(0)\|_{\circledast}$ is sufficiently small, then

$$
\mathscr{E}(0) \leq C\|\mathfrak{X}(0)\|_{\mathbb{1}}^{2}
$$

for some $C>0$. Here the smallness assumption is needed to deal with the nonlinearities in (5-34)(5-35) and the $r$ terms in $\mathscr{E}$. Third, the linear growing modes produced in Theorem 2.1 are in $\mathbb{H}$. It is straightforward to extract the proper definition of $\mathbb{H}$ from $\mathscr{E}$ and to work out the details of the estimate of $\mathscr{E}(0)$; as such, for the sake of brevity, we omit these. With $\mathbb{H}$ defined in this way, it is then easy to use estimate (2-13) of Theorem 2.1 in conjunction with (2-8)-(2-10) to see that the growing modes are in $\mathbb{H}$.

Now that we have stated the nonlinear compatibility conditions, we see why we cannot simply set $\mathfrak{X}(0)=\iota \mathfrak{X}_{0}$ with

$$
\mathfrak{X}_{0}=\left(\begin{array}{c}
\sigma_{\star} \\
\bar{w}_{\star}
\end{array}\right)
$$

for $\sigma_{\star}$ and $v_{\star}=\bar{w}_{\star} / r_{0}^{2}$ the growing mode solution constructed in Theorem 2.1 and $\iota>0$ a small parameter. Indeed, these solve

$$
\lambda \mathfrak{X}_{0}+\mathscr{L} \mathfrak{X}_{0}=0 \text { and } \mathfrak{C}\left(\mathfrak{X}_{0}\right)=0 \quad \Longrightarrow \quad \mathfrak{C}\left(\mathscr{L}^{j} \mathfrak{X}_{0}\right)=0 \quad \text { for all } j \geq 0,
$$


which in particular means that $\mathfrak{X}(0)=\iota \mathfrak{X}_{0}$ does not satisfy the nonlinear compatibility condition (5-36) for $j \geq 1$.

To get around this obstacle, we will use the implicit function theorem to produce a curve of initial data satisfying the compatibility conditions, close to the linear growing modes. To this end, let us define the map $F: \mathbb{H} \rightarrow \mathbb{R}^{12}$ via

$$
F(\mathfrak{X})=\left(\begin{array}{c}
\mathfrak{C}(\mathfrak{X}) \\
\mathfrak{C}\left(\partial_{t} \mathfrak{X}\right) \\
\mathfrak{C}\left(\partial_{t}^{2} \mathfrak{X}\right) \\
\mathfrak{C}\left(\partial_{t}^{3} \mathfrak{X}\right)
\end{array}\right)-\left(\begin{array}{c}
\mathfrak{N}_{\mathfrak{B}_{B}}(\mathfrak{X}) \\
\partial_{t} \mathfrak{N}_{\mathscr{B}}(\mathfrak{X}) \\
\partial_{t}^{2} \mathfrak{N}_{\mathscr{B}_{\mathfrak{B}}}(\mathfrak{X}) \\
\partial_{t}^{3} \mathfrak{N}_{\mathscr{B}}(\mathfrak{X})
\end{array}\right)
$$

where we understand that $\partial_{t}^{j} \mathfrak{X}$ and $\partial_{t}^{j} \mathfrak{N}_{\mathscr{乃}}(\mathfrak{X})$ for $j=1,2,3$ are computed in terms of $\mathfrak{X}$ as in (5-34)-(5-35). Let $\mathfrak{X}_{0}$ be the linear growing modes as above and let $\mathfrak{X}_{i} \in \mathbb{H}, i=1, \ldots, 12$, be arbitrary for now, with exact values to be chosen later. We then define $f: \mathbb{R}^{1+12} \rightarrow \mathbb{R}^{12}$ via

$$
f(t, \tau)=F\left(t \mathfrak{X}_{0}+\sum_{i=1}^{12} \tau_{i} \mathfrak{X}_{i}\right) \quad \text { for } t \in \mathbb{R} \text { and } \tau \in \mathbb{R}^{12} .
$$

Given the structure of the nonlinearities $\mathfrak{N}(\cdot)$ and $\mathfrak{N}_{\mathscr{B}}(\cdot)$, one easily sees that $f \in C^{2}\left(\mathbb{R}^{1+12} ; \mathbb{R}^{12}\right)$. Also, $f(0,0)=0$ and

$$
\frac{\partial f}{\partial t}(0,0)=\left(\begin{array}{c}
\mathfrak{C}\left(\mathfrak{X}_{0}\right) \\
\mathfrak{C}\left(\lambda \mathfrak{X}_{0}\right) \\
\mathfrak{C}\left(\lambda^{2} \mathfrak{X}_{0}\right) \\
\mathfrak{C}\left(\lambda^{3} \mathfrak{X}_{0}\right)
\end{array}\right)=0 \quad \text { and } \quad \frac{\partial f}{\partial \tau_{i}}(0,0)=\left(\begin{array}{c}
\mathfrak{C}\left(\mathfrak{X}_{i}\right) \\
\mathfrak{C}\left(-\mathscr{L} \mathfrak{X}_{i}\right) \\
\mathfrak{C}\left(\mathscr{L}^{2} \mathfrak{X}_{i}\right) \\
\mathfrak{C}\left(-\mathscr{L}^{3} \mathfrak{X}_{i}\right)
\end{array}\right)
$$

From this it is then straightforward to choose the $\mathfrak{X}_{i}$ for $i=1, \ldots, 12$ such that the $12 \times 12$ matrix $(\partial f / \partial \tau)(0,0)$ is invertible. The implicit function theorem then provides a small constant $\iota_{0}>0$ and a function $\xi:\left(-\iota_{0}, \iota_{0}\right) \rightarrow \mathbb{R}^{12}$ such that $f(t, \xi(t))=0$ for all $t \in\left(-\iota_{0}, \iota_{0}\right)$ and such that $\xi \in C^{2}$ and $\xi(0)=0$. We may then differentiate the equation $f(t, \xi(t))=0$ with respect to $t$, set $t=0$, and use the first equation in (5-41) to see that

$$
0=\frac{\partial f}{\partial t}(0,0)+\frac{\partial f}{\partial \tau}(0,0) \frac{d \xi(0)}{d t}=\frac{\partial f}{\partial \tau}(0,0) \frac{d \xi}{d t}(0) \Longrightarrow \frac{d \xi}{d t}(0)=0,
$$

since the matrix $(\partial f / \partial \tau)(0,0)$ is invertible. Then $\xi \in C^{2}$ with $\xi(0)=\dot{\xi}(0)=0$ such that $\xi(t) / t^{2}$ is well-defined and continuous on $\left(-\iota_{0}, \iota_{0}\right)$. Using this, we may then deduce the existence of a small parameter $\iota_{0}>0$ and a curve $\mathfrak{Y}:\left(-\iota_{0}, \iota_{0}\right) \rightarrow \mathbb{H}$ given by

$$
\mathfrak{Y}(\iota)=\iota \mathfrak{X}_{0}+\iota^{2} \sum_{i=1}^{12} \mathfrak{X}_{i} \frac{\xi_{i}(\iota)}{\iota^{2}}:=\iota \mathfrak{X}_{0}+\iota^{2} \overline{\mathfrak{Y}}(\iota),
$$


such that for all $\iota \in\left(-\iota_{0}, \iota_{0}\right)$,

$$
\begin{aligned}
F(\mathfrak{Y}(\iota)) & =0, \text { that is, } \mathfrak{Y}(\iota) \text { satisfies the nonlinear compatibility conditions, } \\
\sqrt{\mathscr{E}(\mathfrak{Y}(\iota))} & \leq C\|\mathfrak{Y}(\iota)\|_{\mathbb{G}} \leq C \iota, \text { and } \\
\mathfrak{E}\left(\overline{\mathfrak{Y}}(\iota)_{1}, \overline{\mathfrak{Y}}(\iota)_{2}\right) & \leq C,
\end{aligned}
$$

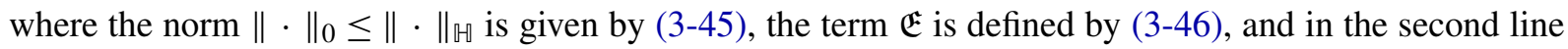
we have written $\mathscr{E}(\mathfrak{Y}(\iota))$ for $\mathscr{E}(0)$ computed from the initial data $\mathfrak{X}(0)=\mathfrak{Y}(\iota)$.

We now recast the above discussion as a lemma.

Lemma 5.3. Let $\sigma_{\star}, v_{\star}$ be the growing mode solution constructed in Theorem 2.1 , write $\bar{w}_{\star}=r_{0}^{2} v_{\star}$, and assume the normalization

$$
\left\|\left(\begin{array}{c}
\sigma_{\star} \\
\bar{w}_{\star}
\end{array}\right)\right\|_{0}=1
$$

for $\|\cdot\|_{0}$ the norm defined by (3-45). Then there exist a number $\iota_{0}>0$ and a family of initial data

$$
\left(\begin{array}{l}
\sigma^{\iota}(0) \\
w^{\iota}(0)
\end{array}\right)=\mathfrak{X}(\iota)=\iota\left(\begin{array}{c}
\sigma_{\star} \\
\bar{w}_{\star}
\end{array}\right)+\iota^{2}\left(\begin{array}{l}
\sigma_{0}(\iota) \\
w_{0}(\iota)
\end{array}\right)
$$

for $\iota \in\left[0, \iota_{0}\right)$ such that the following hold.

(1) $\mathfrak{X}(\iota)$ satisfies the nonlinear compatibility conditions required for a solution to the nonlinear problem (5-32) to exist in the energy space defined by $\mathscr{E}$.

(2) If $\mathscr{E}(0)$ denotes the value of $\mathscr{E}$ determined at $t=0$ from the data $\mathfrak{X}(\iota)$, then $\mathscr{E}(0) \leq C \iota^{2}$ for a constant $C>0$.

(3) For all $\iota \in\left[0, \iota_{0}\right)$, we have

$$
\left\|\left(\begin{array}{c}
\sigma_{0}(\iota) \\
w_{0}(\iota)
\end{array}\right)\right\|_{0}^{2} \leq \mathfrak{E}\left(\sigma_{0}(\iota), \quad w_{0}(\iota)\right) \leq C
$$

for a constant $C>0$ independent of $\iota$, where $\mathfrak{E}$ is given by (3-46).

(4) Let $\psi^{\iota}$ denote the function given by (3-40), with $N_{\mathscr{B}}=N_{\mathscr{B}}(\mathfrak{X}(\iota))$ determined by the data $\mathfrak{X}(\iota)$ at $t=0$. Then $\bar{w}^{\iota}(0)=w^{\iota}(0)-\psi^{\iota}$ satisfies the homogeneous boundary condition $\mathscr{B}\left(\bar{w}^{\imath}(0)\right)=0$ and

$$
\left\|\left(\begin{array}{c}
0 \\
\psi^{\iota}
\end{array}\right)\right\|_{0}^{2} \leq \mathfrak{E}\left(0, \psi^{\iota}\right) \leq C \iota^{4}
$$

for a constant $C>0$ independent of $\iota$.

Proof. Everything except for the last item is proved above. The last item follows from Lemma 3.4 and the fact that $N_{\mathscr{B}}$ is at least a quadratic nonlinearity. 
5D. Instability. We are now ready to prove our main result.

Theorem 5.4. There exist $\theta_{0}>0, C>0$, and $0<\iota_{0}<\theta_{0}$ such that for any $0<\iota \leq \iota_{0}$, there exists a family of solutions $\sigma^{\iota}(t)$ and $v^{\iota}(t)$ to the Navier-Stokes-Poisson system (4-2) such that

$$
\sqrt{\mathscr{E}}(0) \leq C \iota, \quad \text { but } \quad \sup _{0 \leq t \leq T^{\iota}} \sqrt{\mathscr{E} 0}(t) \geq \sup _{0 \leq t \leq T^{\iota}} \sqrt{\mathscr{\mathscr { E }} 0, \sigma^{\iota}\left(T^{\iota}\right)+\mathscr{\mathscr { E }} 0, v^{\iota}\left(T^{\iota}\right)} \geq \theta_{0} .
$$

Here $T^{\iota}$ is given by $T^{\iota}=\frac{1}{\lambda} \ln \frac{\theta_{0}}{\iota}$ and $\mathscr{E}^{0, \sigma^{\iota}}$ and $\mathscr{E}^{0, v^{\iota}}$ are defined in the first line of (4-11).

Proof. We divide the proof into steps. At several points in the proof we will restrict the size of $\theta$. Whenever we do so, we assume that $\iota$ is also restricted such that $0<\iota \leq \iota_{0} \leq \theta$. We will choose the value of $\theta_{0}$ in the final step of the proof.

Step 1 (data and the solutions). Let us assume that $\iota_{0}$ is as small as the $\iota_{0}$ appearing in Lemma 5.3, and then let $\mathfrak{X}(\iota)$ for $\iota \leq \iota_{0}$ be the family of initial data for the nonlinear problem (5-32)-(5-33) given in the lemma. For $0<\iota \leq \iota_{0}$, we now let $\left(\begin{array}{c}\sigma^{\iota} \\ w^{\iota}\end{array}\right)$ be solutions to the Navier-Stokes-Poisson system (5-32)-(5-33) with a family of initial data

$$
\left.\left(\begin{array}{c}
\sigma^{\iota} \\
w^{\iota}
\end{array}\right)\right|_{t=0}=\left(\begin{array}{l}
\sigma^{\iota}(0) \\
w^{\iota}(0)
\end{array}\right)=\mathfrak{X}(\iota)=\iota\left(\begin{array}{c}
\sigma_{\star} \\
\bar{w}_{\star}
\end{array}\right)+\iota^{2}\left(\begin{array}{c}
\sigma_{0}(\iota) \\
w_{0}(\iota)
\end{array}\right) .
$$

The solution satisfies $\sqrt{\mathscr{E}}(0) \leq C \iota$.

Note that since

$$
r^{\iota}(x, 0)=\left(\frac{3}{4 \pi} \int_{0}^{x} \frac{d y}{\rho_{0}(y)+\iota \sigma_{*}(y)+\iota^{2} \sigma_{0}(\iota)(y)}\right)^{1 / 3},
$$

a Taylor expansion and item (2) of Lemma 5.3 allow us to estimate

$$
\left\|1-\frac{r_{0}(x)}{r^{\iota}(x, 0)}\right\|_{L^{\infty}}^{2}+\frac{v}{2}\left\|1-\frac{r_{0}(x)}{r^{l}(x, 0)}\right\|_{L^{2}}^{2} \leq A_{1} \iota^{2}
$$

for a constant $A_{1}>0$ independent of $\iota$. From this, the normalization (5-45), and the estimate (5-47), we may assume that $\iota<\iota_{0}$ with $\iota_{0}$ small enough that

$$
\frac{\iota}{2} \leq \sqrt{\mathscr{E} 0, \sigma^{\iota}(0)+\mathscr{\mathscr { E }} 0, v^{\iota}(0)+\mathscr{E}^{1, v^{\iota}}(0)}+\sqrt{\mathscr{\mathscr { E }} 0, r^{\iota}(0)} \leq 2 \iota .
$$

Throughout the rest of the proof we will let $\mathscr{E}(t)$ denote the total energy, defined by (4-19), associated to the solutions $\sigma^{\iota}$ and $w^{\iota}$ at time $t$.

Step 2 (control of the energy). Let us define the constant

$$
B_{0}:=\left(2+\frac{27}{8 \sqrt{2} \lambda}\left\|\rho_{0}\right\|_{L^{\infty}}^{1 / 2}\right)
$$

It will be useful in determining the time-scale in which instability begins. Indeed, we define $T$ by

$$
T:=\sup \left\{s \mid \sqrt{\mathscr{E} 0, \sigma^{l}(t)+\mathscr{\mathscr { E }} 0, v^{l}(t)+\mathscr{\mathscr { E }} 1, v^{l}(t)}+\sqrt{\mathscr{E} 0, r^{l}(t)} \leq\left(4+B_{0}\right) \iota e^{\lambda t} \text { for } 0 \leq t \leq s\right\} .
$$


The estimate (5-53) guarantees that $T>0$. Then by Proposition 5.1 and (5-53), there exist $C_{\star}$ and $\theta_{\star}>0$ such that for $0 \leq t \leq \min \left\{T, T\left(\iota, \theta_{\star}\right)\right\}$ (with $T\left(\iota, \theta_{\star}\right)$ given in the proposition),

$$
\sqrt{\mathscr{E}}(t) \leq C_{\star} l e^{\lambda t} .
$$

Let us assume that $\theta \leq \theta_{\star}$, which means that $T^{\iota} \leq T\left(\iota, \theta_{\star}\right)$, and hence that the estimate (5-56) also holds for $0 \leq t \leq \min \left\{T, T^{\imath}\right\}$. Let us further assume that $\theta$ is small enough that $\sqrt{\mathscr{E}}(t) \leq C_{\star} \theta$ is small enough that the right side of the estimate in Lemma 4.9 is smaller than $\frac{1}{2}$. In particular, this implies that

$$
\left\|\frac{\sigma^{l}(t)}{\rho_{0}}\right\|_{L^{\infty}}+\left\|1-\frac{r_{0}}{r^{l}(t)}\right\|_{L^{\infty}} \leq \frac{1}{2}
$$

for all $0 \leq t \leq \min \left\{T, T^{\iota}\right\}$. By further restricting $\theta$ to decrease the bound of the terms in (5-57), and using the identities in (4-22) and (4-23), we can also bound

$$
\frac{1}{4}\left(\mathscr{E}^{0, \sigma^{l}}(t)+\mathscr{E}^{0, v^{l}}(t)+\mathscr{E}^{1, v^{l}}(t)\right) \leq\left\|\left(\begin{array}{c}
\sigma^{l}(t) \\
w^{l}(t)
\end{array}\right)\right\|_{0}^{2} \leq 2\left(\mathscr{E}^{0, \sigma^{l}}(t)+\mathscr{E}^{0, v^{l}}(t)+\mathscr{E}^{1, v^{l}}(t)\right)
$$

for $0 \leq t \leq T^{\iota}=\min \left\{T, T^{\iota}\right\}$.

Step 3 (linear estimates for $\sigma^{\iota}$ and $w^{\iota}$ ). Because of the estimate (5-57), the boundary condition $w^{\iota} /\left.\left(r^{\iota}\right)^{2}\right|_{x=0}$ is equivalent to $w^{\iota} /\left.r_{0}^{2}\right|_{x=0}$. We can then modify the problem (5-32)-(5-33) to have the form (3-41)(3-42), the latter of which has the homogeneous boundary conditions (3-42). This leads us to consider $\bar{w}^{\iota}(0)=w^{\iota}(0)-\psi^{\iota}$ as in Lemma 5.3, which satisfies $\mathscr{B}\left(\bar{w}^{\iota}(0)\right)=0$ at $x=M$. We then have that

$$
e^{t \mathscr{L}}\left(\begin{array}{c}
\sigma^{\iota}(0) \\
\bar{w}^{\iota}(0)
\end{array}\right)=\imath e^{\lambda t}\left(\begin{array}{c}
\sigma_{\star} \\
\bar{w}_{\star}
\end{array}\right)+\iota^{2} e^{t \mathscr{L}}\left(\begin{array}{c}
\sigma_{0}(\iota) \\
w_{0}(\iota)
\end{array}\right)-e^{t \mathscr{L}}\left(\begin{array}{c}
0 \\
\psi^{\iota}
\end{array}\right) .
$$

Then the solutions $\left(\begin{array}{c}\sigma^{\iota} \\ w^{\iota}\end{array}\right)$ to (5-32) can be written as in (3-44):

$$
\begin{aligned}
\left(\begin{array}{c}
\sigma^{l}(t) \\
w^{\iota}(t)
\end{array}\right)=\iota e^{\lambda t}\left(\begin{array}{c}
\sigma_{\star} \\
\bar{w}_{\star}
\end{array}\right)+\iota^{2} e^{t \mathscr{L}}\left(\begin{array}{c}
\sigma_{0}(l) \\
w_{0}(l)
\end{array}\right)-e^{t \mathscr{L}}\left(\begin{array}{c}
0 \\
\psi^{\iota}
\end{array}\right)-\frac{1}{\delta}\left(\begin{array}{c}
0 \\
N_{\mathscr{P}}^{\iota}(t) r_{0}^{3} / 3
\end{array}\right) & \\
& +\int_{0}^{t} e^{(t-s) \mathscr{L}}\left(\begin{array}{c}
N_{1}^{\iota}(s) \\
N_{2}^{\iota}(s)
\end{array}\right) d s+\frac{1}{\delta} \int_{0}^{t} e^{(t-s) \mathscr{L}}\left(\begin{array}{c}
N_{\mathscr{\beta}}^{\iota}(s) \rho_{0} \\
\partial_{t} N_{\mathscr{\beta}}^{\iota}(s) r_{0}^{3} / 3
\end{array}\right) d s .
\end{aligned}
$$

Here the nonlinear terms $N_{\mathscr{R}}^{\iota}, N_{1}^{\iota}$, and $N_{2}^{\iota}$ are defined in terms of $w^{\iota}$ and $\sigma^{\iota}$ via (5-22) and (5-23).

Theorem 3.5, together with the nonlinear estimates of Lemma 5.2, imply that if the inequality $t \leq$ $\min \left\{T, T\left(\iota, \theta_{\star}\right)\right\}$ holds, then

$$
\begin{aligned}
\left\|\left(\begin{array}{c}
\sigma^{\iota}(t) \\
w^{l}(t)
\end{array}\right)-\iota e^{t \lambda}\left(\begin{array}{c}
\sigma_{\star} \\
\bar{w}_{\star}
\end{array}\right)-\iota^{2} e^{t \mathscr{L}}\left(\begin{array}{c}
\sigma_{0}(\iota) \\
w_{0}(\iota)
\end{array}\right)+e^{t \mathscr{L}}\left(\begin{array}{c}
0 \\
\psi^{l}
\end{array}\right)\right\|_{0} & \leq C|\mathscr{E}(t)|^{2}+C \int_{0}^{t} e^{\lambda(t-s) \mathscr{E}(s) d s} \\
& \leq C\left(\iota e^{\lambda t}\right)^{2}+C \int_{0}^{t} e^{\lambda(t-s)} \iota^{2} e^{2 \lambda s} d s \\
& \leq A_{2}\left(\iota e^{\lambda t}\right)^{2}
\end{aligned}
$$


for a constant $A_{2}>0$ independent of $\iota$. On the other hand, because of the estimates (3-47) and (5-47)(5-48), we may estimate

$$
\begin{aligned}
\left\|\iota^{2} e^{t \mathscr{L}}\left(\begin{array}{c}
\sigma_{0}(\iota) \\
w_{0}(\iota)
\end{array}\right)\right\|_{0}+\left\|e^{t \mathscr{L}}\left(\begin{array}{c}
0 \\
\psi^{\iota}
\end{array}\right)\right\|_{0} & \leq \iota^{2} C e^{\lambda t} \sqrt{\mathfrak{E}\left(\sigma_{0}(\iota), w_{0}(\iota)\right)}+C e^{\lambda t} \sqrt{\mathfrak{E}\left(0, \psi^{\iota}\right)} \\
& \leq \iota^{2} A_{3} e^{\lambda t}
\end{aligned}
$$

for a constant $A_{3}>0$ independent of $\iota$. Then we may then deduce from (5-61)-(5-62) and the normalization (5-45) that

$$
\left\|\left(\begin{array}{c}
\sigma^{\iota}(t) \\
w^{\iota}(t)
\end{array}\right)\right\|_{0} \leq \iota e^{\lambda t}+\iota^{2} A_{3} e^{\lambda t}+A_{2}\left(\iota e^{\lambda t}\right)^{2} .
$$

Step 4 (control of the $r$ energy). We now turn to control of the term $\mathscr{E}^{0, r^{r}}$. First note that

$$
\frac{d}{d t} \int \frac{v}{2}\left|1-\frac{r_{0}}{r^{\iota}}\right|^{2} d x \leq\left(\int v\left|1-\frac{r_{0}}{r^{\iota}}\right|^{2} d x\right)^{1 / 2}\left(\int v\left|\frac{w^{\iota}}{r_{0}^{3}}\right|^{2} d x\right)^{1 / 2}\left\|\frac{r_{0}}{r^{\iota}}\right\|_{L^{\infty}}^{4},
$$

which together with (5-57) implies that

$$
\frac{d}{d t} \sqrt{\mathscr{E}^{0, r^{l}}(t)} \leq \frac{81}{16 \sqrt{2}}\left(\int v\left|\frac{w^{\iota}}{r_{0}^{3}}\right|^{2} d x\right)^{1 / 2}
$$

We may then argue as in (4-23) to see that

$$
\int \frac{v}{\rho_{0}}\left|\frac{w^{\iota}}{r_{0}^{3}}\right|^{2} d x \leq \int \frac{32 \pi^{2}}{9}\left(\delta \rho_{0}\left|\partial_{x} w^{\iota}\right|^{2}+\frac{4 \varepsilon}{3} \rho_{0} r_{0}^{6}\left|\partial_{x}\left(\frac{w^{\iota}}{r_{0}^{3}}\right)\right|^{2}\right) d x \leq \frac{4}{9}\left\|\left(\begin{array}{c}
\sigma^{\iota}(t) \\
w^{\iota}(t)
\end{array}\right)\right\|_{0}^{2} .
$$

Combining (5-65) and (5-66) with (5-63), we find that

$$
\frac{d}{d t} \sqrt{\mathscr{E} 0, r^{l}(t)} \leq \frac{27}{8 \sqrt{2}}\left\|\rho_{0}\right\|_{L^{\infty}}^{1 / 2}\left\|\left(\begin{array}{c}
\sigma^{\iota}(t) \\
w^{\iota}(t)
\end{array}\right)\right\|_{0} \leq \frac{27}{8 \sqrt{2}}\left\|\rho_{0}\right\|_{L^{\infty}}^{1 / 2}\left(\iota e^{\lambda t}+A_{3} \iota^{2} e^{\lambda t}+A_{2}\left(\iota e^{\lambda t}\right)^{2}\right)
$$

for $0 \leq t \leq \min \left\{T, T^{\iota}\right\}$. Integrating this from 0 to $t \leq \min \left\{T, T^{\iota}\right\}$ and employing (5-53) then yields the estimate

$$
\begin{aligned}
\sqrt{\mathscr{E}^{0, r^{l}}(t)} & \leq\left(2+\frac{27}{8 \sqrt{2} \lambda}\left\|\rho_{0}\right\|_{L^{\infty}}^{1 / 2}\right)\left(\iota e^{\lambda t}+A_{3} \iota^{2} e^{\lambda t}\right)+\frac{27 A_{2}}{16 \sqrt{2} \lambda}\left\|\rho_{0}\right\|_{L^{\infty}}^{1 / 2}\left(\iota e^{\lambda t}\right)^{2} \\
& =B_{0}\left(\iota e^{\lambda t}\right)+\left(\iota A_{4}\right)\left(\iota e^{\lambda t}\right)+A_{5}\left(\iota e^{\lambda t}\right)^{2}
\end{aligned}
$$

for $0 \leq t \leq \min \left\{T, T^{\iota}\right\}$, where $B_{0}$ is the constant defined above in (5-54) and $A_{4}, A_{5}$ are constants independent of $\iota$.

Step 5 (the bound $\left.T^{\iota} \leq T\right)$. We now claim that if $\theta$ is taken to be small enough, then $T^{\iota}=(1 / \lambda) \ln (\theta / \iota) \leq T$. Suppose by way of contradiction that $T^{\iota}>T$. Then the first bounds in (5-58), (5-63), and (5-68) imply that

$$
\begin{aligned}
\sqrt{\mathscr{E} 0, \sigma^{\imath}(t)+\mathscr{E} 0, v^{l}(t)+\mathscr{E} 1, v^{\imath}(t)}+\sqrt{\mathscr{E} 0, r^{\imath}(t)} & \leq\left(2+B_{0}+\iota A_{4}\right)\left(\iota e^{\lambda t}\right)+\left(2 A_{2}+A_{5}\right)\left(\iota e^{\lambda t}\right)^{2} \\
& \leq\left[2+B_{0}+\iota A_{4}+\left(2 A_{2}+A_{5}\right) \theta\right]\left(\iota e^{\lambda t}\right) \\
& \leq\left[3+B_{0}\right]\left(\iota e^{\lambda t}\right)
\end{aligned}
$$


for $t \leq T^{\iota}$, if we assume that $\theta$ is small enough that $\theta\left(2 A_{2}+A_{5}\right) \leq \frac{1}{2}$ and $\iota_{0} A_{4} \leq \frac{1}{2}$. For this choice of $\theta$, we then find from the definition of $T$ that $T \geq T^{\iota}$, a contradiction. Hence, $T^{\iota} \leq T$ for $\theta$ sufficiently small. Step 6 (conclusion: instability). We now define the $L^{2}$ part of the norm $\|\cdot\|_{0}$ by

$$
\left\|\left(\begin{array}{c}
\sigma \\
w
\end{array}\right)\right\|_{00}^{2}:=\frac{1}{2} \int K \gamma \rho_{0}^{\gamma-1}\left|\frac{\sigma}{\rho_{0}}\right|^{2} d x+\frac{1}{2} \int\left|\frac{w}{r_{0}^{2}}\right|^{2} d x .
$$

Note that $\|\cdot\|_{00} \leq\|\cdot\|_{0}$ and that by the normalization (5-45), we have that the data satisfy

$$
\left\|\left(\begin{array}{c}
\sigma_{\star} \\
\bar{w}_{\star}
\end{array}\right)\right\|_{00}^{2}:=C_{00} \in(0,1) \text {. }
$$

Also, we may argue as in the derivation of (5-58) to see that

$$
\sqrt{\mathscr{E} 0, \sigma^{\iota}(t)+\mathscr{\mathscr { C }} 0, v^{l}(t)} \geq \frac{1}{\sqrt{2}}\left\|\left(\begin{array}{c}
\sigma^{\iota} \\
w^{\iota}
\end{array}\right)\right\|_{00}
$$

for $0 \leq t \leq T^{\iota}=\min \left\{T, T^{\iota}\right\}$.

Let us now further assume that $\theta$ is small enough that $A_{2} \theta \leq \frac{C_{00}}{4}$ and $\iota_{0} A_{3} \leq \frac{C_{00}}{4}$. We can then combine (5-71), (5-72), (5-61), and (5-62) to deduce that

$$
\begin{aligned}
& \sqrt{\mathscr{\mathscr { E }} 0, \sigma^{\iota}\left(T^{\iota}\right)+\mathscr{E} 0, v^{\iota}\left(T^{\iota}\right)} \geq \frac{1}{\sqrt{2}}\left\|\left(\begin{array}{c}
\sigma^{\iota}\left(T^{\iota}\right) \\
w^{\iota}\left(T^{\iota}\right)
\end{array}\right)\right\|_{00} \geq \frac{1}{\sqrt{2}} l e^{\lambda T^{\iota}}\left\|\left(\begin{array}{c}
\sigma_{\star} \\
\bar{w}_{\star}
\end{array}\right)\right\|_{00} \\
& -\frac{1}{\sqrt{2}}\left\|\left(\begin{array}{c}
\sigma^{\iota}\left(T^{\iota}\right) \\
w^{\iota}\left(T^{\iota}\right)
\end{array}\right)-\iota e^{\lambda T^{\iota}}\left(\begin{array}{c}
\sigma_{\star} \\
\bar{w}_{\star}
\end{array}\right)-\iota^{2} e^{T^{\iota} \mathscr{L}}\left(\begin{array}{c}
\sigma_{0}(\iota) \\
w_{0}(\iota)
\end{array}\right)+e^{T^{\iota} \mathscr{L}}\left(\begin{array}{c}
0 \\
\psi^{\iota}
\end{array}\right)\right\|_{00} \\
& -\frac{1}{\sqrt{2}}\left\|\iota^{2} e^{T^{\prime} \mathscr{L}}\left(\begin{array}{c}
\sigma_{0}(\iota) \\
w_{0}(\iota)
\end{array}\right)\right\|_{00}-\frac{1}{\sqrt{2}}\left\|e^{T^{\prime} \mathscr{L}}\left(\begin{array}{c}
0 \\
\psi^{l}
\end{array}\right)\right\|_{00} \\
& \geq \frac{1}{\sqrt{2}} \iota e^{\lambda T^{\iota}} C_{00}-\frac{1}{\sqrt{2}}\left\|\iota^{2} e^{T^{\imath} \mathscr{L}}\left(\begin{array}{c}
\sigma_{0}(\iota) \\
w_{0}(\iota)
\end{array}\right)\right\|_{0}-\frac{1}{\sqrt{2}}\left\|e^{T^{\prime} \mathscr{L}}\left(\begin{array}{c}
0 \\
\psi^{\iota}
\end{array}\right)\right\|_{0} \\
& -\frac{1}{\sqrt{2}}\left\|\left(\begin{array}{c}
\sigma^{\iota}\left(T^{\iota}\right) \\
w^{\iota}\left(T^{\iota}\right)
\end{array}\right)-\iota e^{\lambda T^{\iota}}\left(\begin{array}{c}
\sigma_{\star} \\
\bar{w}_{\star}
\end{array}\right)-\iota^{2} e^{T^{\iota} \mathscr{L}}\left(\begin{array}{c}
\sigma_{0}(\iota) \\
w_{0}(\iota)
\end{array}\right)+e^{T^{\iota} \mathscr{L}}\left(\begin{array}{c}
0 \\
\psi^{\iota}
\end{array}\right)\right\|_{0} \\
& \geq \frac{1}{\sqrt{2}}\left(\iota e^{\lambda T^{\iota}} C_{00}-A_{3} \iota^{2} e^{\lambda T^{\iota}}-A_{2}\left(\iota e^{\lambda T^{\iota}}\right)^{2}\right) \\
& =\frac{1}{\sqrt{2}}\left(\theta C_{00}-\theta \iota A_{3}-A_{2} \theta^{2}\right) \geq \frac{C_{00}}{2 \sqrt{2}} \theta \text {. }
\end{aligned}
$$

Setting $\theta_{0}=\frac{\theta C_{00}}{2 \sqrt{2}}$, we find that (5-49) holds. This completes the proof of the theorem.

\section{References}

[Chandrasekhar 1939] S. Chandrasekhar, An introduction to the study of stellar structure, University of Chicago, Chicago, 1939. Reprinted Dover, New York, 1957. MR 19,1142b Zbl 0022.19207

[Deng et al. 2002] Y. Deng, T.-P. Liu, T. Yang, and Z.-a. Yao, "Solutions of Euler-Poisson equations for gaseous stars", Arch. Ration. Mech. Anal. 164:3 (2002), 261-285. MR 2004h:35187 Zbl 1038.76036 
[Ducomet and Zlotnik 2005] B. Ducomet and A. Zlotnik, "Stabilization and stability for the spherically symmetric NavierStokes-Poisson system", Appl. Math. Lett. 18:10 (2005), 1190-1198. MR 2006b:35259 Zbl 1125.35071

[Guo and Strauss 1995] Y. Guo and W. A. Strauss, "Instability of periodic BGK equilibria", Comm. Pure Appl. Math. 48:8 (1995), 861-894. MR 96j:35252 Zbl 0840.45012

[Guo and Tice 2010] Y. Guo and I. Tice, "Linear Rayleigh-Taylor instability for viscous, compressible fluids", SIAM J. Math. Anal. 42:4 (2010), 1688-1720. MR 2011j:76084 Zbl 05895002

[Jang 2008] J. Jang, "Nonlinear instability in gravitational Euler-Poisson systems for $\gamma=\frac{6}{5}$ ”, Arch. Ration. Mech. Anal. 188:2 (2008), 265-307. MR 2009a:85003 Zbl 1192.85003

[Jang 2010] J. Jang, "Local well-posedness of dynamics of viscous gaseous stars", Arch. Ration. Mech. Anal. 195:3 (2010), 797-863. MR 2011c:35453 Zbl 1197.35294

[Jang and Masmoudi 2009] J. Jang and N. Masmoudi, "Well-posedness for compressible Euler equations with physical vacuum singularity", Comm. Pure Appl. Math. 62:10 (2009), 1327-1385. MR 2010j:35384 Zbl 1213.35298

[Jang and Masmoudi 2010] J. Jang and N. Masmoudi, "Well-posedness of compressible Euler equations in a physical vacuum", preprint, 2010. arXiv 1005.4441

[Lin 1997] S.-S. Lin, "Stability of gaseous stars in spherically symmetric motions", SIAM J. Math. Anal. 28:3 (1997), 539-569. MR 98b:85001 Zbl 0871.35012

[Matusu-Necasova et al. 1997] S. Matusu-Necasova, M. Okada, and T. Makino, "Free boundary problem for the equation of spherically symmetric motion of viscous gas, III", Japan J. Indust. Appl. Math. 14:2 (1997), 199-213. MR 98e:35177

[Okada and Makino 1993] M. Okada and T. Makino, "Free boundary problem for the equation of spherically symmetric motion of viscous gas", Japan J. Indust. Appl. Math. 10:2 (1993), 219-235. MR 94f:35153 Zbl 0783.76082

[Rein 2003] G. Rein, "Non-linear stability of gaseous stars", Arch. Ration. Mech. Anal. 168:2 (2003), 115-130. MR 2005d:35214 Zbl 1044.76026

[Zhang and Fang 2009] T. Zhang and D. Fang, "Global behavior of spherically symmetric Navier-Stokes-Poisson system with degenerate viscosity coefficients”, Arch. Ration. Mech. Anal. 191:2 (2009), 195-243. MR 2010f:35447 Zbl 1161.76051

Received 5 Jul 2012. Revised 4 Jan 2013. Accepted 28 Feb 2013.

JUHI JANG: juhijang@math.ucr.edu

Department of Mathematics, University of California, Riverside, Riverside, CA 92521, United States

IAN TICE: iantice@andrew. cmu.edu

Department of Mathematical Sciences, Carnegie Mellon University, Pittsburgh, PA 15213, United States 


\title{
Analysis \& PDE
}

\author{
msp.org/apde
}

\section{EDITORS}

EDITOR-IN-CHIEF

\author{
Maciej Zworski \\ zworski@math.berkeley.edu \\ University of California \\ Berkeley, USA
}

BOARD OF EDITORS

Nicolas Burq Université Paris-Sud 11, France

nicolas.burq@math.u-psud.fr

Sun-Yung Alice Chang Princeton University, USA

chang@math.princeton.edu

Michael Christ University of California, Berkeley, USA

mchrist@math.berkeley.edu

Charles Fefferman Princeton University, USA

cf@math.princeton.edu

Ursula Hamenstaedt Universität Bonn, Germany

ursula@math.uni-bonn.de

Vaughan Jones U.C. Berkeley \& Vanderbilt University vaughan.f.jones@vanderbilt.edu

Herbert Koch Universität Bonn, Germany koch@math.uni-bonn.de

Izabella Laba University of British Columbia, Canada ilaba@math.ubc.ca

Gilles Lebeau Université de Nice Sophia Antipolis, France lebeau@unice.fr

László Lempert Purdue University, USA lempert@math.purdue.edu

Richard B. Melrose Massachussets Institute of Technology, USA rbm@math.mit.edu

Frank Merle Université de Cergy-Pontoise, France Frank.Merle@u-cergy.fr

William Minicozzi II Johns Hopkins University, USA minicozz@math.jhu.edu

Werner Müller Universität Bonn, Germany mueller@math.uni-bonn.de
Yuval Peres

Gilles Pisier

Tristan Rivière

Igor Rodnianski

Wilhelm Schlag

Sylvia Serfaty

Yum-Tong Siu

Terence Tao

Michael E. Taylor

Gunther Uhlmann

András Vasy

Dan Virgil Voiculescu

Steven Zelditch
University of California, Berkeley, USA

peres@stat.berkeley.edu

Texas A\&M University, and Paris 6

pisier@math.tamu.edu

ETH, Switzerland

riviere@math.ethz.ch

Princeton University, USA

irod@math.princeton.edu

University of Chicago, USA

schlag@math.uchicago.edu

New York University, USA

serfaty@cims.nyu.edu

Harvard University, USA

siu@math.harvard.edu

University of California, Los Angeles, USA

tao@math.ucla.edu

Univ. of North Carolina, Chapel Hill, USA

met@math.unc.edu

University of Washington, USA

gunther@math.washington.edu

Stanford University, USA

andras@math.stanford.edu

University of California, Berkeley, USA

dvv@math.berkeley.edu

Northwestern University, USA

zelditch@math.northwestern.edu

\section{PRODUCTION}

production@msp.org

Silvio Levy, Scientific Editor

See inside back cover or msp.org/apde for submission instructions.

The subscription price for 2013 is US \$160/year for the electronic version, and \$310/year ( $\$ 35$, if shipping outside the US) for print and electronic. Subscriptions, requests for back issues from the last three years and changes of subscribers address should be sent to MSP.

Analysis \& PDE (ISSN 1948-206X electronic, 2157-5045 printed) at Mathematical Sciences Publishers, 798 Evans Hall \#3840, c/o University of California, Berkeley, CA 94720-3840, is published continuously online. Periodical rate postage paid at Berkeley, CA 94704, and additional mailing offices.

APDE peer review and production are managed by EditFLOW ${ }^{\circledR}$ from Mathematical Sciences Publishers.

PUBLISHED BY

- mathematical sciences publishers

nonprofit scientific publishing

http://msp.org/

(C) 2013 Mathematical Sciences Publishers 


\section{ANALYSIS \& PDE}

\section{Volume $6 \quad$ No. $5 \quad 2013$}

A Lichnerowicz estimate for the first eigenvalue of convex domains in Kähler manifolds

1001

VINCENT GUEDJ, BORIS KOLEV and NADER YEGANEFAR

Sharp modulus of continuity for parabolic equations on manifolds and lower bounds for the 1013 first eigenvalue

Ben ANDREws and Julie ClutTERbuCK

Some minimization problems in the class of convex functions with prescribed determinant

NAM Q. LE and OVIDIU SAVIN

On the spectrum of deformations of compact double-sided flat hypersurfaces

1051

DENIS BORISOV and PEDRO FREITAS

Stabilization for the semilinear wave equation with geometric control condition

1089

ROMAIN JOLY and CAMILLE LAURENT

Instability theory of the Navier-Stokes-Poisson equations

JUHI JANG and IAN TICE

Dynamical ionization bounds for atoms

1183

ENNO LENZMANN and MATHIEU LEWIN

Nodal count of graph eigenfunctions via magnetic perturbation

GREGORY BERKOLAIKO

Magnetic interpretation of the nodal defect on graphs

YVES COLIN DE VERDIÈRE 